Supporting Information

\title{
Enhancement of Mechanophore Activation in Mechanochromic Dendrimers by Functionalization of Their Surface
}

Takuma Watabe, Daisuke Aoki, and Hideyuki Otsuka*

Department of Chemical Science and Engineering, Tokyo Institute of Technology, 2-12-1 Ookayama, Meguro-ku, Tokyo 152-8550, Japan

Table of Contents

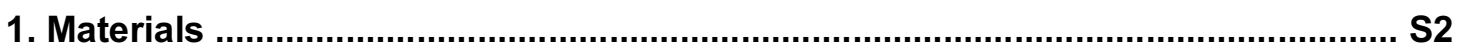

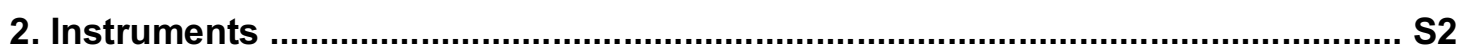

3. Grinding tests and electron paramagnetic resonance (EPR) spectroscopy....... S2

4. Synthesis and Characterization

4.1. Azidation and surface modification of $\left(\mathrm{EtO}_{2} \mathrm{C}\right) 2^{\mathrm{n}}$-[G-n]-Br............................ 3

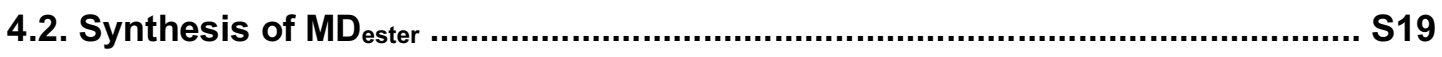

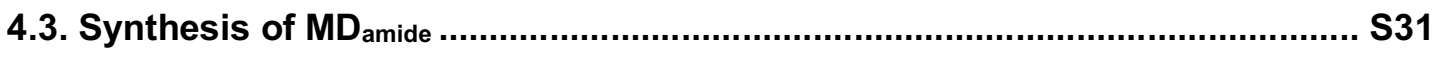

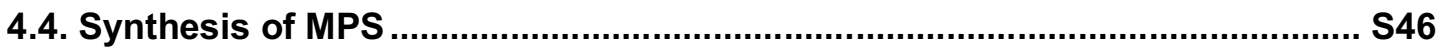

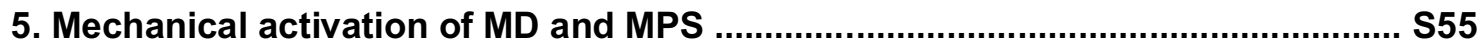




\section{Materials}

All solvents and reagents were purchased from Sigma-Aldrich, Wako Pure Chemical Industries, Tokyo Chemical Industry, and Kanto Chemical and used as received, unless otherwise noted. DABBF-dialkyne, DABBF-tetraalkyne ${ }^{1}$ and ester surfaced Fréchet-type dendrons $\left(\left(\mathbf{E t O}_{2} \mathbf{C}\right)_{2}{ }^{n}-[\mathbf{G}-\mathbf{n}]-\mathbf{B r}\right)^{2}$ were synthesized

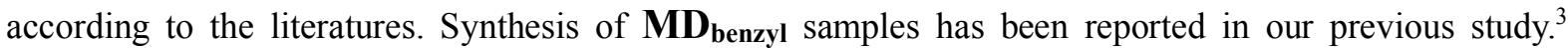
Copper(I) bromide $(\mathrm{CuBr})$ was washed with acetic acid and with methanol, and dried in vacuo.

\section{Instruments}

${ }^{1} \mathrm{H}$ and ${ }^{13} \mathrm{C}$ NMR spectra were recorded on a Bruker AVANCE III HD500 spectrometer. IR spectra were recorded on a JEOL FT/IR-4100 Fourier transform infrared spectrometer as thin films with KBr or cast on $\mathrm{NaCl}$. UV-vis absorption spectroscopy was performed on a JASCO V-650 spectrophotometer with an integrating-sphere unit at r.t. Analytical gel permeation chromatographic (GPC) measurements were carried out at $40{ }^{\circ} \mathrm{C}$ on TOSOH HLC-8320 GPC system (eluent: THF) or JASCO ChromNAV Lite system (eluent: DMF), each equipped with a guard column (TOSOH TSK guard column Super H-L), three columns (TOSOH TSK gel SuperH 6000, 4000, and 2500), a differential refractive index detector, and a UV-vis detector. A flow rate of the eluent was $0.6 \mathrm{~mL} / \mathrm{min}$. MALDI-TOF MS spectrometry was performed on a SHIMADZU AXIMA Performance MALDI-TOF mass spectrometer operating in linear mode or Bruker ultrafleXtreme, using dithranol or trans-2-[3-(4-tert-butylphenyl)-2-methyl-2-propenylidene]malononitrile (DCTB) in combination with sodium trifluoroacetate (NaTFA). ESI-TOF MS spectrometry was performed on Bruker Daltonics micrOTOF II . DSC measurements were carried out using a SHIMADZU DSC-60A Plus with a heating rate $10{ }^{\circ} \mathrm{C} / \mathrm{min}$. Dynamic light scattering (DLS) measurements were performed at $25^{\circ} \mathrm{C}$ on Otsuka Electronics ELSZ-2000. The scattering angle for the detector was $164.5^{\circ}$.

\section{Grinding tests and electron paramagnetic resonance (EPR) spectroscopy}

Grinding tests were performed on a Retsch Mixer Mill MM 400. The applied force was controlled by vibrational frequency and grinding time. A powdered sample $(50 \mathrm{mg})$ was placed in the grinding jar and ground for $10 \mathrm{~min}$ at $30 \mathrm{~Hz}$, unless otherwise stated. The ground samples were transferred into an EPR 5 $\mathrm{mm}$ glass capillary, and the capillary was sealed after being degassed. EPR measurements were carried out on a JEOL JES-X320 X-band EPR spectrometer equipped with a JEOL DVT temperature controller. The spectra of ground samples were measured using a microwave power of $0.199 \mathrm{~mW}$, field modulation of 0.1 $\mathrm{mT}$ with a time constant of $0.03 \mathrm{~s}$, a sweep rate of $0.125 \mathrm{mT} / \mathrm{s}$ at $25^{\circ} \mathrm{C}$, and integrated for 16 times. The amount of the radicals formed from the cleavage of DABBF was determined by comparing the area of the observed integral spectrum with a $0.05 \mathrm{mM}$ solution of 4-hydroxy-2,2,6,6-tetramethylpiperidine-1-oxyl (TEMPOL) in benzene under the same experimental condition. The $\mathrm{Mn}^{2+}$ signal was used as an auxiliary standard. The $g$ value was calculated according to the following equation: $g=h v / \beta H$ where $h$ is the Planck constant, $v$ is the microwave frequency, $\beta$ is the Bohr magneton, and $H$ is the magnetic field. 


\section{Synthesis and Characterization}

\subsection{Azidation and surface modification of $\left(\mathrm{EtO}_{2} \mathrm{C}\right){ }_{2}{ }^{\mathrm{n}}-[\mathrm{G}-\mathrm{n}]-\mathrm{Br}$}

$\left(\mathrm{EtO}_{2} \mathrm{C}\right)_{2}$-[G-1]-N 3
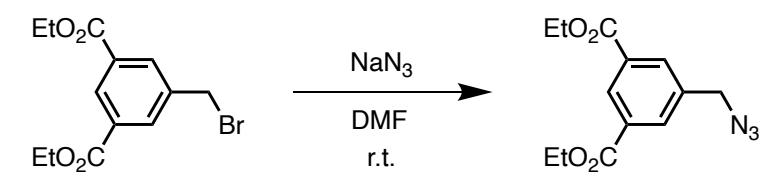

$\left(\mathrm{EtO}_{2} \mathrm{C}\right)_{2}-[\mathrm{G}-1]-\mathrm{Br}$

$\left(\mathrm{EtO}_{2} \mathrm{C}\right)_{2}-[\mathrm{G}-1]-\mathrm{N}_{3}$

Under a nitrogen atmosphere, $\left(\mathbf{E t O}_{\mathbf{2}} \mathbf{C}\right)_{2}-[\mathbf{G}-\mathbf{1}]-\mathbf{B r}(3.00 \mathrm{~g}, 9.53 \mathrm{mmol})$ and sodium azide $(1.46 \mathrm{~g}, 28.6 \mathrm{mmol})$ in anhydrous DMF $(30 \mathrm{~mL})$ were stirred at r.t. for 1 day. The reaction mixture was precipitated into water. Following filtration, the product was dried in vacuo to afford $\left(\mathbf{E t O}_{2} \mathbf{C}\right)_{2}-[\mathbf{G}-1]-\mathbf{N}_{3}$ as a white solid $(2.55 \mathrm{~g}$, 97\%).

${ }^{1} \mathrm{H}$ NMR (500 MHz, $\left.\mathrm{CDCl}_{3}\right): \delta / \mathrm{ppm} 8.65$ (t, $J=1.6 \mathrm{~Hz}, 1 \mathrm{H}$, aromatic), 8.19 (d, $J=1.6 \mathrm{~Hz}, 2 \mathrm{H}$, aromatic), $4.49\left(\mathrm{~s}, 2 \mathrm{H}, \mathrm{CH}_{2}\right), 4.43\left(\mathrm{q}, J=7.1 \mathrm{~Hz}, 4 \mathrm{H}, \mathrm{CH}_{2}\right), 1.43\left(\mathrm{t}, J=7.1 \mathrm{~Hz}, 6 \mathrm{H}, \mathrm{CH}_{3}\right) ;{ }^{13} \mathrm{C}$ NMR $(125 \mathrm{MHz}, \mathrm{DMSO}-$ $\left.d_{6}\right): \delta / \mathrm{ppm} 165.43,136.45,133.15,131.58,130.44,61.60,53.95,14.35$; FT-IR $\left(\mathrm{KBr}, \mathrm{cm}^{-1}\right): 3072,2982$, 2943, 2093, 1707, 1476, 1372, 1319, 1241, 1198, 1028, 876, 760, 715.

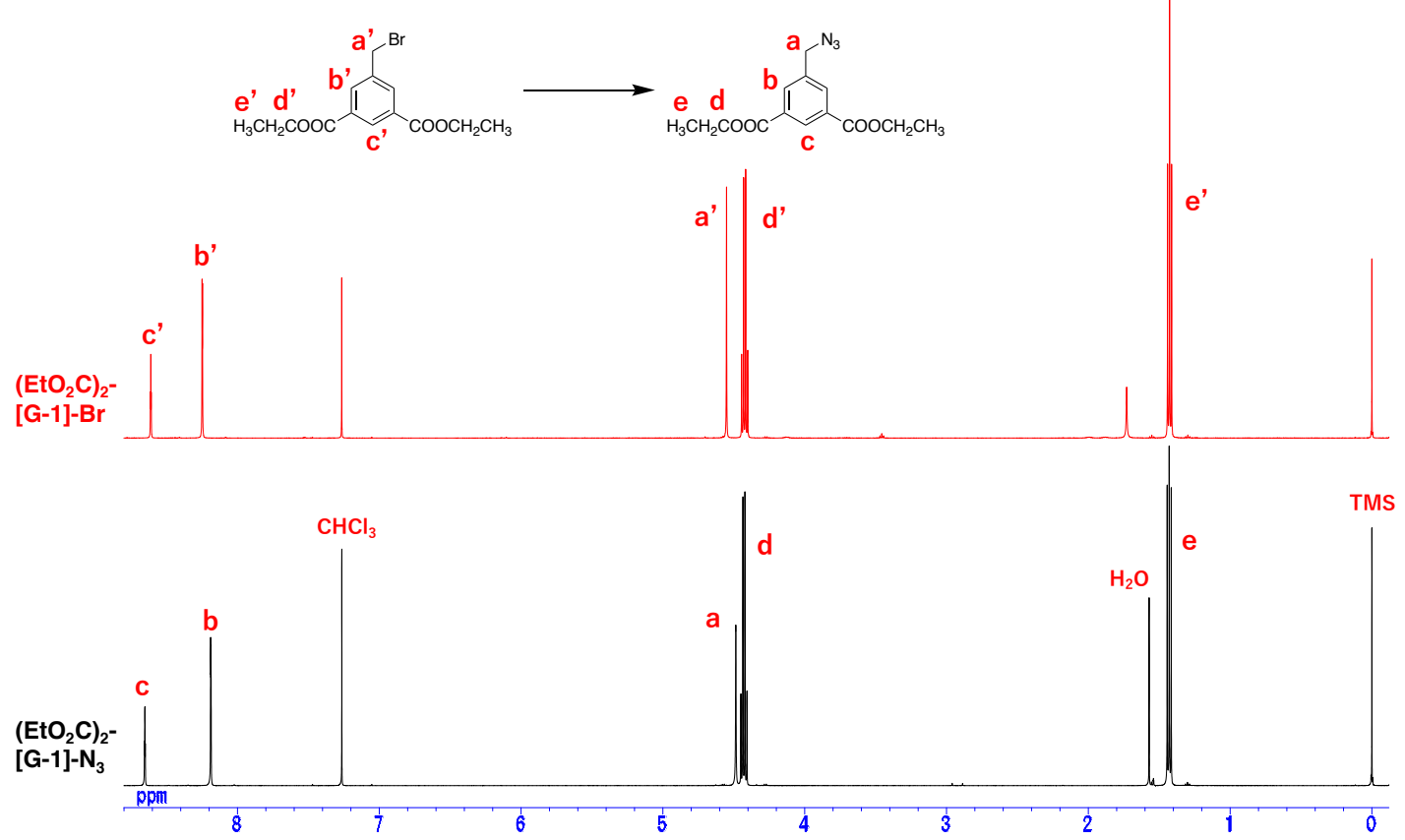

Figure S1. ${ }^{1} \mathrm{H}$ NMR spectra of $\left(\mathbf{E t O}_{2} \mathbf{C}\right)_{2}-[\mathbf{G}-\mathbf{1}]-\mathbf{N}_{3}$ (black) and $\left(\mathbf{E t O}_{2} \mathbf{C}\right)_{2}-[\mathbf{G}-\mathbf{1}]-\mathbf{B r}$ (red) $\left(500 \mathrm{MHz}, \mathrm{CDCl}_{3}\right)$. 
$\left(\mathrm{HO}_{2} \mathrm{C}\right)_{2}-[\mathrm{G}-1]-\mathrm{N}_{3}$

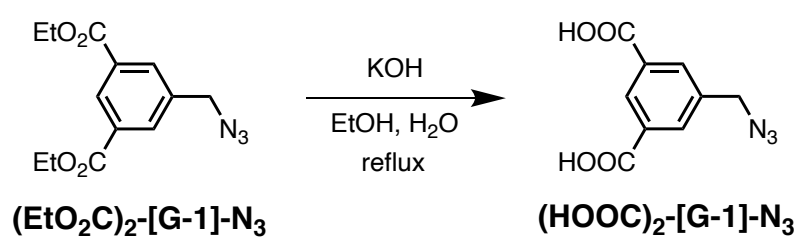

$\left(\mathbf{E t O}_{2} \mathbf{C}\right)_{2}$-[G-1]-N $\mathbf{N}_{3}(0.961 \mathrm{~g}, 3.47 \mathrm{mmol})$ and potassium hydroxide $(1.99 \mathrm{~g}, 35.5 \mathrm{mmol})$ were combined with EtOH $(20 \mathrm{~mL})$ and water $(5 \mathrm{~mL})$ in a round-bottom flask equipped with a reflux condenser. The mixture was refluxed for $1 \mathrm{~h}$. After evaporation, the resulting solution was neutralized with $1 \mathrm{M} \mathrm{HCl}$, at which point a white precipitate formed. Following filtration, the product was dried in vacuo to afford $\left(\mathrm{HO}_{2} \mathbf{C}\right)_{2}-[\mathbf{G}-\mathbf{1}]-\mathbf{N}_{3}$ as a white solid $(0.712 \mathrm{~g}, 93 \%)$.

${ }^{1} \mathrm{H}$ NMR (500 MHz, DMSO- $d_{6}$ ): $\delta / \mathrm{ppm} 13.42$ (s, 2H, COOH), 8.44 (s, $1 \mathrm{H}$, aromatic), 8.17 (s, 2H, aromatic), $4.68\left(\mathrm{~s}, 2 \mathrm{H}, \mathrm{CH}_{2}\right) ;{ }^{13} \mathrm{C}$ NMR (125 MHz, DMSO- $\left.d_{6}\right): \delta / \mathrm{ppm} 166.80,137.74,133.50,132.16,129.99,52.99$; FT-IR $\left(\mathrm{KBr}, \mathrm{cm}^{-1}\right)$ : 2988, 2097, 1701, 1461, 1281, 693, 487.
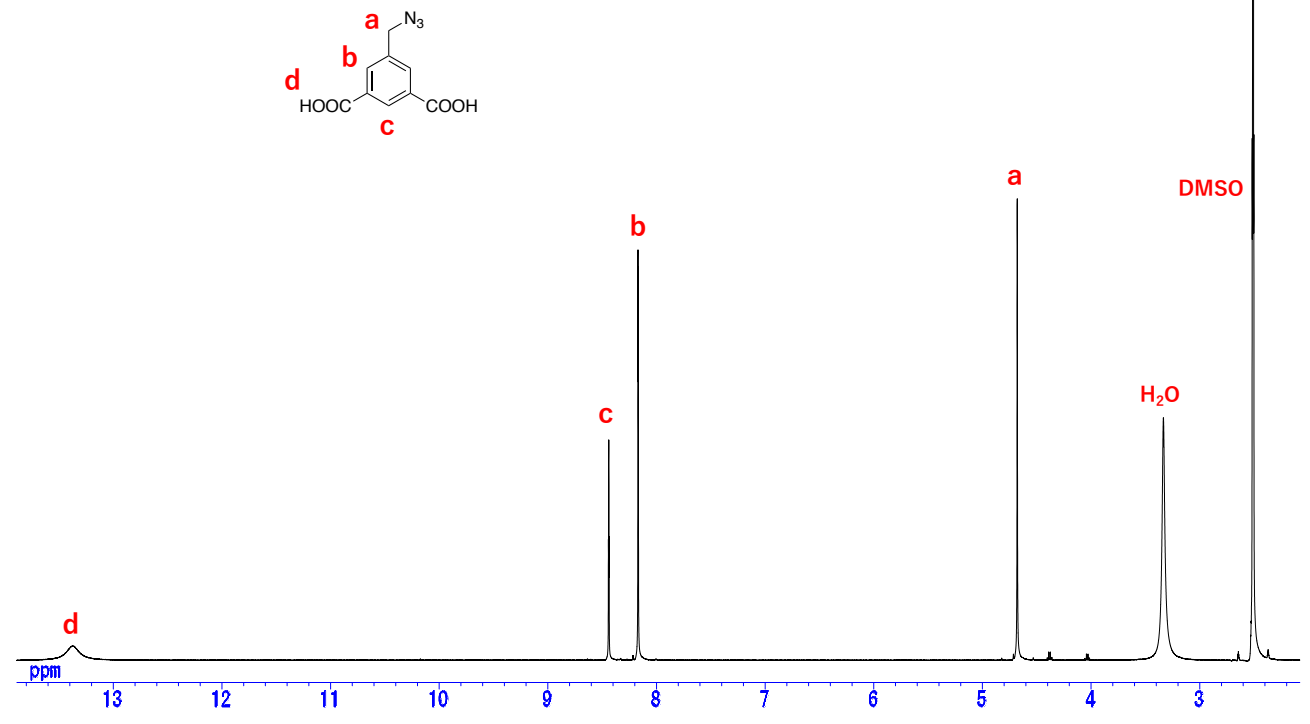

Figure S2. ${ }^{1} \mathrm{H}$ NMR spectrum of (HOOC) $)_{2}-[\mathbf{G}-1]-\mathbf{N}_{\mathbf{3}}\left(500 \mathrm{MHz}, \mathrm{DMSO}-d_{6}\right)$. 
$\left(\mathrm{H}_{2} \mathrm{NOC}\right)_{2}-[\mathrm{G}-1]-\mathrm{N}_{3}$
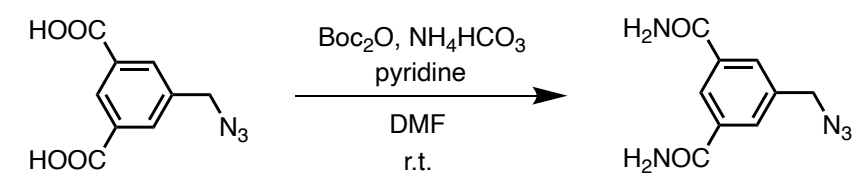

$(\mathrm{HOOC})_{2}-[\mathrm{G}-1]-\mathrm{N}_{3}$

$\left(\mathrm{H}_{2} \mathrm{NOC}\right)_{2}-[\mathrm{G}-1]-\mathrm{N}_{3}$

To a solution of $\left(\mathbf{H O}_{2} \mathbf{C}\right)_{2}-[\mathbf{G}-1]-\mathbf{N}_{3}(1.00 \mathrm{~g}, 4.52 \mathrm{mmol})$, pyridine $(0.728 \mathrm{~mL}, 9.04 \mathrm{mmol})$, and di-tert-butyl dicarbonate $(2.99 \mathrm{~g}, 13.7 \mathrm{mmol})$ in anhydrous DMF $(15 \mathrm{~mL})$ was added ammonium bicarbonate $(1.08 \mathrm{~g}$, $13.7 \mathrm{mmol}$ ) and the mixture was allowed to stir at r.t. for $15 \mathrm{~h}$. The resultant mixture was poured into water and cooled to $0{ }^{\circ} \mathrm{C}$. After several hours, white precipitate formed. Following filtration, the product was dried in vacuo $t$ to afford $\left(\mathbf{H}_{2} \mathbf{N O C}\right)_{2}$-[G-1]- $\mathbf{N}_{3}$ as a pale yellow solid $(0.712 \mathrm{~g}, 72 \%)$.

${ }^{1} \mathrm{H}$ NMR (500 MHz, DMSO- $d_{6}$ ): $\delta /$ ppm 8.33 (s, 1H, aromatic), 8.07 (s, 2H, NH), 7.99 (s, 2H, aromatic), 7.50 (s, 2H, NH), 4.59 (s, $\left.2 \mathrm{H}, \mathrm{CH}_{2}\right) ;{ }^{13} \mathrm{C}$ NMR (125 MHz, DMSO- $d_{6}$ ): $\delta / \mathrm{ppm} 167.25,136.25,134.96$, 130.01, 126.32, 53.18; FT-IR (KBr, cm $\left.{ }^{-1}\right): 3856,3397,3174,2097,1685,1618,1588,1444,1382,1246$, $1132,871,779,623,418$.
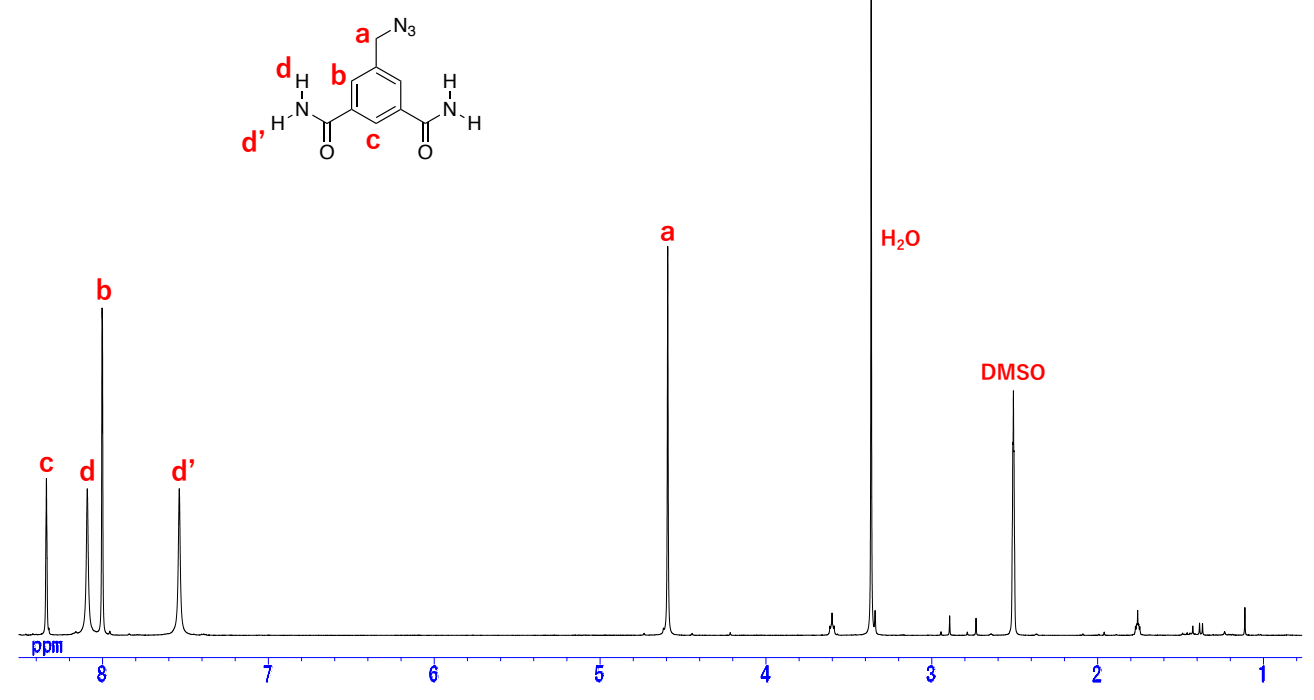

Figure S3. ${ }^{1} \mathrm{H}$ NMR spectrum of $\left(\mathbf{H}_{2} \mathbf{N O C}\right)_{2}-[\mathbf{G}-1]-\mathbf{N}_{3}\left(500 \mathrm{MHz}, \mathrm{DMSO}-d_{6}\right)$. 


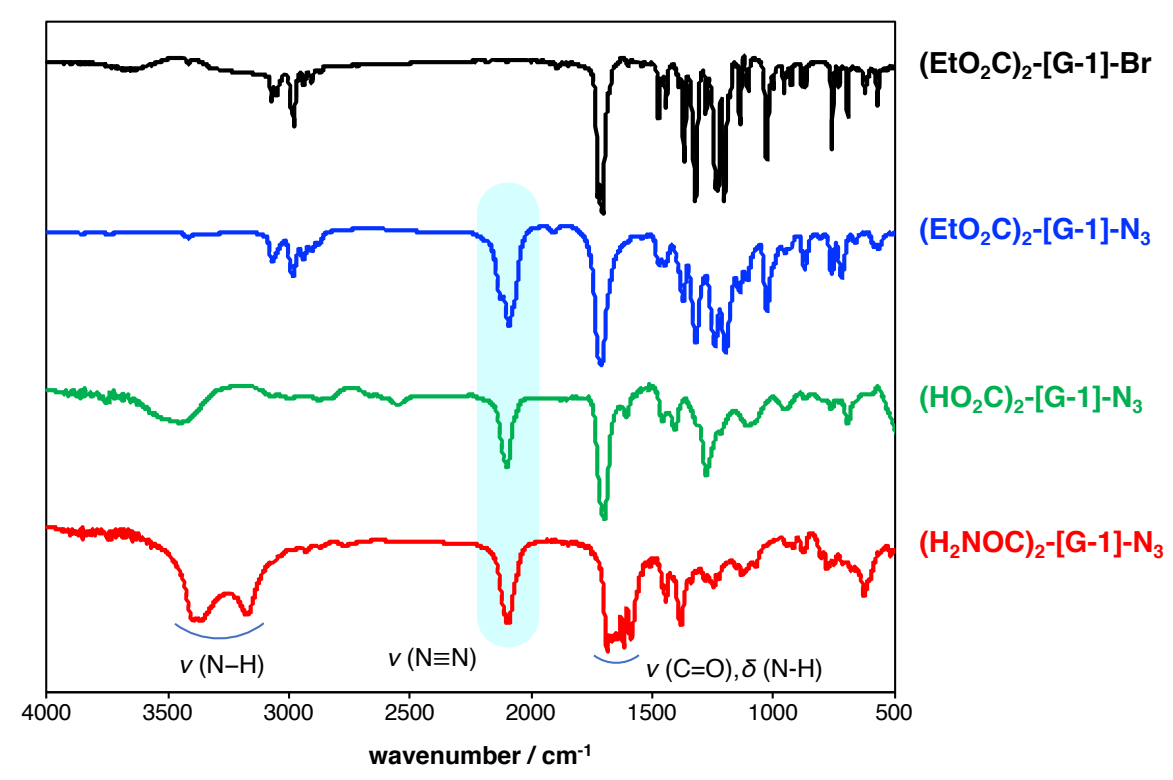

Figure S4. IR spectra of $(\mathbf{R})_{2}-[G-1]-X(K B r)$.

$\left(\mathrm{EtO}_{2} \mathrm{C}\right)_{4-[\mathrm{G}-2]-\mathrm{N}_{3}}$

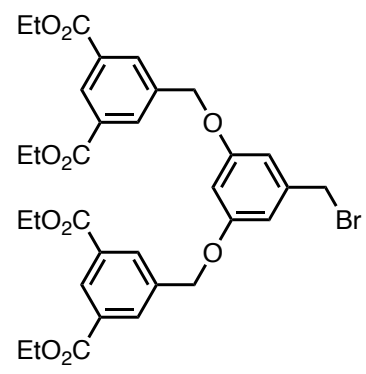

$\left(\mathrm{EtO}_{2} \mathrm{C}\right)_{4}-[\mathrm{G}-2]-\mathrm{Br}$

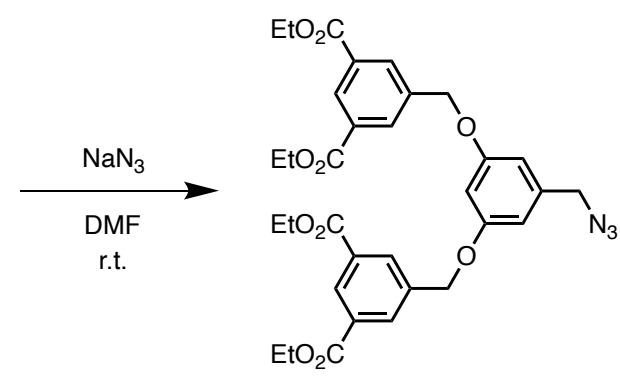

$\left(\mathrm{EtO}_{2} \mathrm{C}\right)_{4}-[\mathrm{G}-2]-\mathrm{N}_{3}$

$\left(\right.$ EtO $\left._{2} \mathbf{C}\right){ }_{4}$-[G-2]-Br (3.04 g, $\left.4.53 \mathrm{mmol}\right)$ and sodium azide (1.46 g, $\left.22.4 \mathrm{mmol}\right)$ in anhydrous DMF (30 mL) were stirred at r.t. for 1 day. The reaction mixture was precipitated into water. Following filtration, the product was dried in vacuo to afford $\left(\mathbf{E t O}_{2} \mathbf{C}\right)_{4}-[\mathbf{G}-2]-\mathbf{N}_{3}$ as a white solid $(2.72 \mathrm{~g}, 95 \%)$.

${ }^{1} \mathrm{H} \mathrm{NMR} \mathrm{(500} \mathrm{MHz,} \mathrm{CDCl}_{3}$ ): $\delta / \mathrm{ppm} 8.65$ (s, 2H, aromatic), 8.31 (d, $J=1.2 \mathrm{~Hz}, 4 \mathrm{H}$, aromatic), 6.62 (t, $J=$ $2.0 \mathrm{~Hz}, 1 \mathrm{H}$, aromatic), 6.60 (d, $J=2.0 \mathrm{~Hz}, 2 \mathrm{H}$, aromatic), $5.14\left(\mathrm{~s}, 4 \mathrm{H}, \mathrm{CH}_{2}\right), 4.43$ (q, $J=7.2 \mathrm{~Hz}, 8 \mathrm{H}, \mathrm{CH}_{2}$ ), 4.30 (s, $\left.2 \mathrm{H}, \mathrm{CH}_{2}\right), 1.43$ (t, $\left.\left.J=7.1 \mathrm{~Hz}, 12 \mathrm{H}, \mathrm{CH}_{3}\right) ;{ }^{13} \mathrm{C} \mathrm{NMR} \mathrm{(125} \mathrm{MHz,} \mathrm{CDCl}_{3}\right): \delta / \mathrm{ppm} \mathrm{165.61,} \mathrm{159.89,}$ 138.01, 137.53, 132.61, 131.37, 130.32, 107.37, 101.86, 69.13, 61.53, 54.72, 14.36; FT-IR (KBr, cm $\left.{ }^{-1}\right): 2987$, 2102, 1720, 1597, 1442, 1361, 1309, 1240, 1200, 1107, 1071, 1026, 831, 751. 


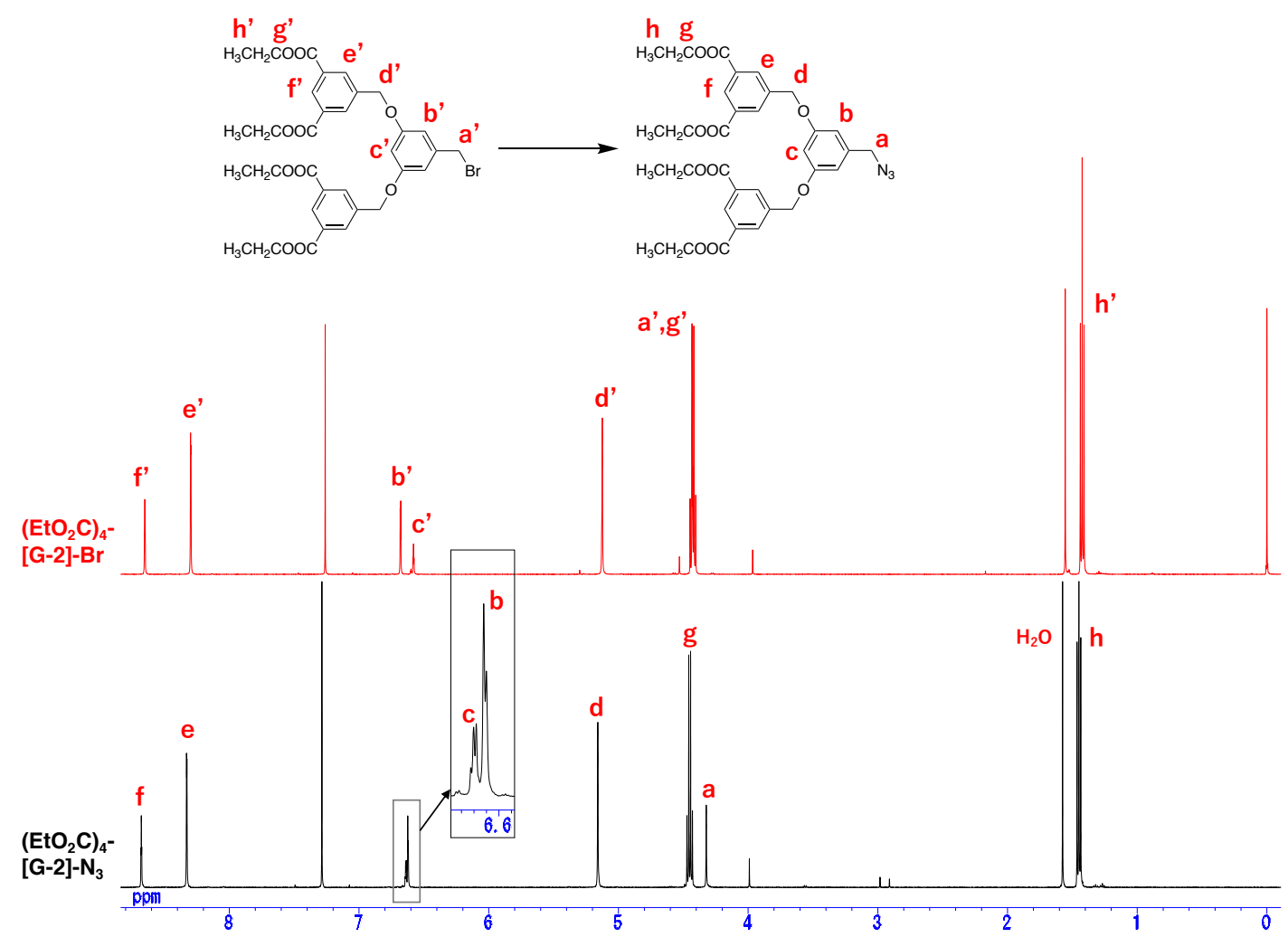

Figure S5. ${ }^{1} \mathrm{H}$ NMR spectra of $\left(\mathbf{E t O}_{2} \mathbf{C}\right)_{4}-[\mathbf{G}-2]-\mathbf{N}_{3}$ (black) and $\left(\mathbf{E t O}_{2} \mathbf{C}\right)_{4}-[\mathbf{G}-2]-\mathbf{B r}$ (red) $\left(500 \mathrm{MHz}, \mathrm{CDCl}_{3}\right)$.

\section{$\left(\mathrm{HO}_{2} \mathrm{C}\right)_{4}-[\mathrm{G}-2]-\mathrm{N}_{3}$}
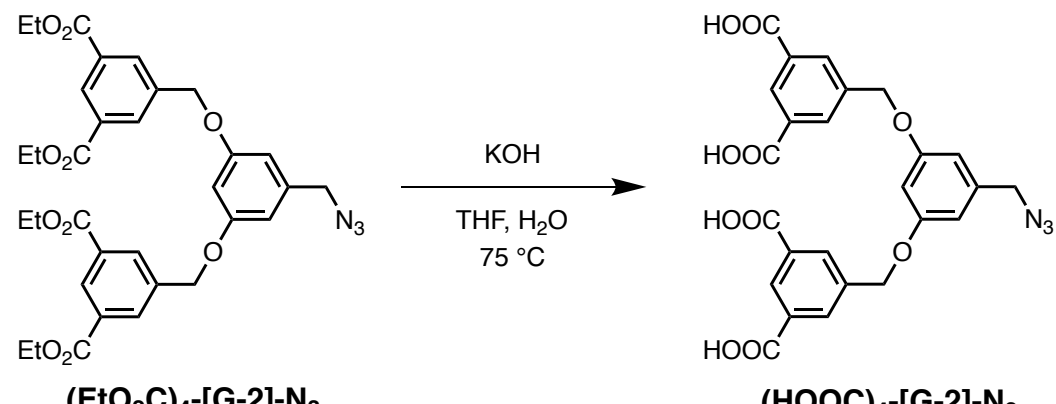

$\left(\mathrm{EtO}_{2} \mathrm{C}\right)_{4}-[\mathrm{G}-2]-\mathrm{N}_{3}$

$(\mathrm{HOOC})_{4}-[\mathrm{G}-2]-\mathrm{N}_{3}$

$\left(\mathbf{E t O}_{2} \mathbf{C}\right)_{4}-[\mathbf{G}-2]-\mathbf{N}_{3}(1.20 \mathrm{~g}, 1.90 \mathrm{mmol})$ and potassium hydroxide $(2.16 \mathrm{~g}, 38.5 \mathrm{mmol})$ were combined with THF $(20 \mathrm{~mL})$ and water $(10 \mathrm{~mL})$ in a round-bottom flask equipped with a reflux condenser. The mixture was heated to $75^{\circ} \mathrm{C}$ for $17 \mathrm{~h}$. After cooling to room temperature, the resulting solution was neutralized with $1 \mathrm{M}$ $\mathrm{HCl}$, at which point a white precipitate formed. Following filtration, the product was dried in vacuo to afford $\left(\mathbf{H O}_{2} \mathbf{C}\right)_{4}-[\mathbf{G}-2]-\mathbf{N}_{3}$ as a white solid (1.00 g, quant.).

${ }^{1} \mathrm{H}$ NMR (500 MHz, DMSO- $d_{6}$ ): $\delta / \mathrm{ppm} 8.43$ (t, $J=1.5 \mathrm{~Hz}, 2 \mathrm{H}$, aromatic), $8.24(\mathrm{~d}, J=1.4 \mathrm{~Hz}, 4 \mathrm{H}$, aromatic), $6.77\left(\mathrm{t}, J=2.1 \mathrm{~Hz}, 1 \mathrm{H}\right.$, aromatic), $6.70\left(\mathrm{~d}, J=2.1 \mathrm{~Hz}, 2 \mathrm{H}\right.$, aromatic), $5.28\left(\mathrm{~s}, 4 \mathrm{H}, \mathrm{CH}_{2}\right), 4.38\left(\mathrm{~s}, 2 \mathrm{H}, \mathrm{CH}_{2}\right)$; ${ }^{13} \mathrm{C}$ NMR (125 MHz, DMSO- $\left.d_{6}\right): \delta /$ ppm 166.91, 159.88, 138.84, 138.57, 132.62, 131.94, 129.85, 108.03, 101.89, 68.72, 53.92; FT-IR (KBr, $\left.\mathrm{cm}^{-1}\right): 2989,2103,1700,1599,1451,1242,1171,1070,898,832,756$, $667,486,468,412$. 

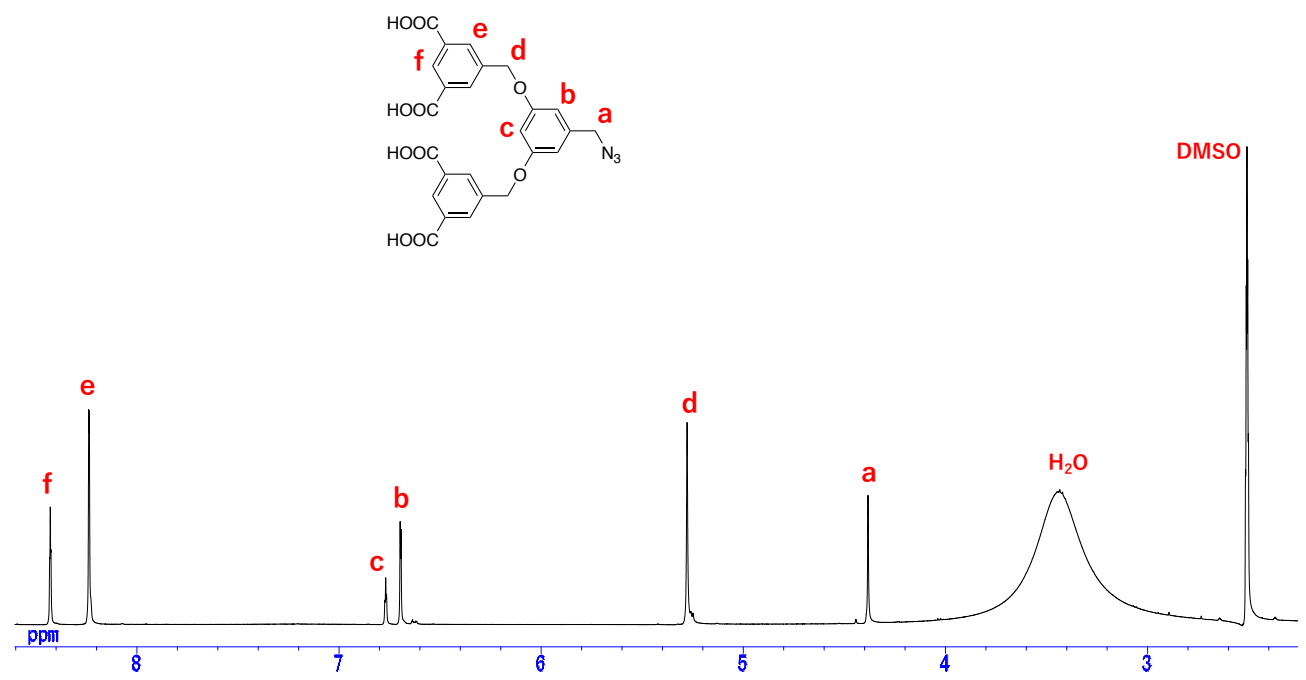

Figure S6. ${ }^{1} \mathrm{H}$ NMR spectrum of $(\mathbf{H O O C})_{4}-[\mathbf{G}-2]-\mathbf{N}_{3}\left(500 \mathrm{MHz}\right.$, DMSO- $\left.d_{6}\right)$.

\section{$\left(\mathrm{H}_{2} \mathrm{NOC}\right)_{4}-[\mathrm{G}-2]-\mathrm{N}_{3}$}
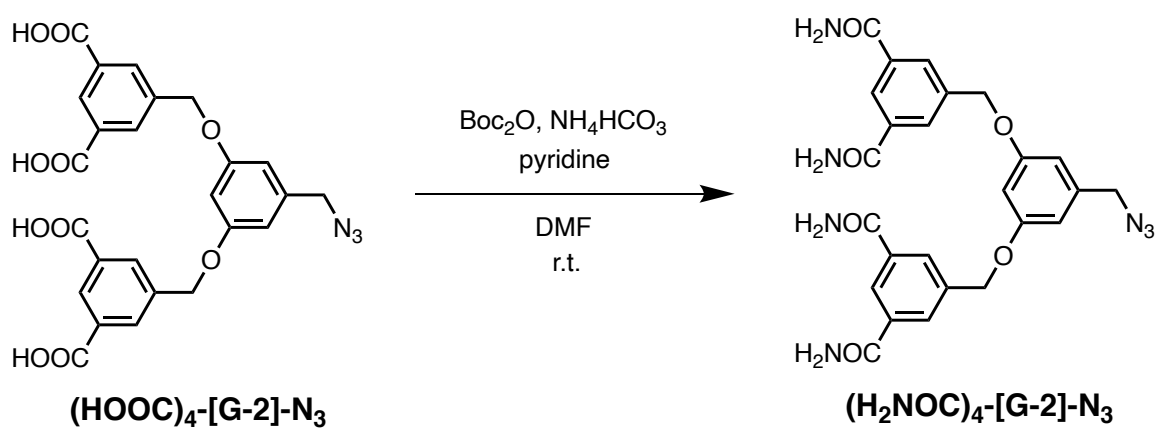

$\left(\mathrm{H}_{2} \mathrm{NOC}\right)_{4}-[\mathrm{G}-2]-\mathrm{N}_{3}$

To a solution of $\left(\mathbf{H O}_{2} \mathbf{C}\right)_{4}-[\mathbf{G}-2]-\mathbf{N}_{3}(0.255 \mathrm{~g}, 0.489 \mathrm{mmol})$, pyridine $(0.155 \mathrm{~mL}, 1.92 \mathrm{mmol})$, and di-tertbutyl dicarbonate $(0.649 \mathrm{~g}, 2.97 \mathrm{mmol})$ in anhydrous DMF $(15 \mathrm{~mL})$ was added ammonium bicarbonate $(0.235 \mathrm{~g}, 2.97 \mathrm{mmol})$ and the mixture was allowed to stir at r.t. for $17 \mathrm{~h}$. The resultant mixture was poured into water. Following filtration, the product was dried in vacuo at $40{ }^{\circ} \mathrm{C}$ overnight to afford $\left(\mathbf{H}_{2} \mathbf{N O C}\right)_{4}$-[G2]-N $\mathbf{N}_{3}$ as a white solid (0.248 g, 98\%).

${ }^{1} \mathrm{H}$ NMR (500 MHz, DMSO- $d_{6}$ ): $\delta /$ ppm 8.33 (s, 2H, aromatic), 8.09 (d, $J=1.4 \mathrm{~Hz}, 4 \mathrm{H}$, aromatic), 8.07 (s, 4H, NH), 7.51 (s, 4H, NH), 6.75 (t, $J=2.2 \mathrm{~Hz}, 1 \mathrm{H}$, aromatic), 6.70 (d, $J=2.1 \mathrm{~Hz}, 2 \mathrm{H}$, aromatic), 5.19 (s, $4 \mathrm{H}, \mathrm{CH}_{2}$ ), 4.40 (s, 2H, $\mathrm{CH}_{2}$ ).; ${ }^{13} \mathrm{C}$ NMR (100 MHz, DMSO- $\left.d_{6}\right): \delta / \mathrm{ppm} 167.56,159.65,138.17,137.42$, 134.79, 129.52, 126.31, 107.64, 101.63, 69.04, 53.66; FT-IR (KBr, cm$\left.{ }^{-1}\right): 3192,2100,1656,1591,1443$, $1378,1239,1165,1074,882,761,631,572$. 


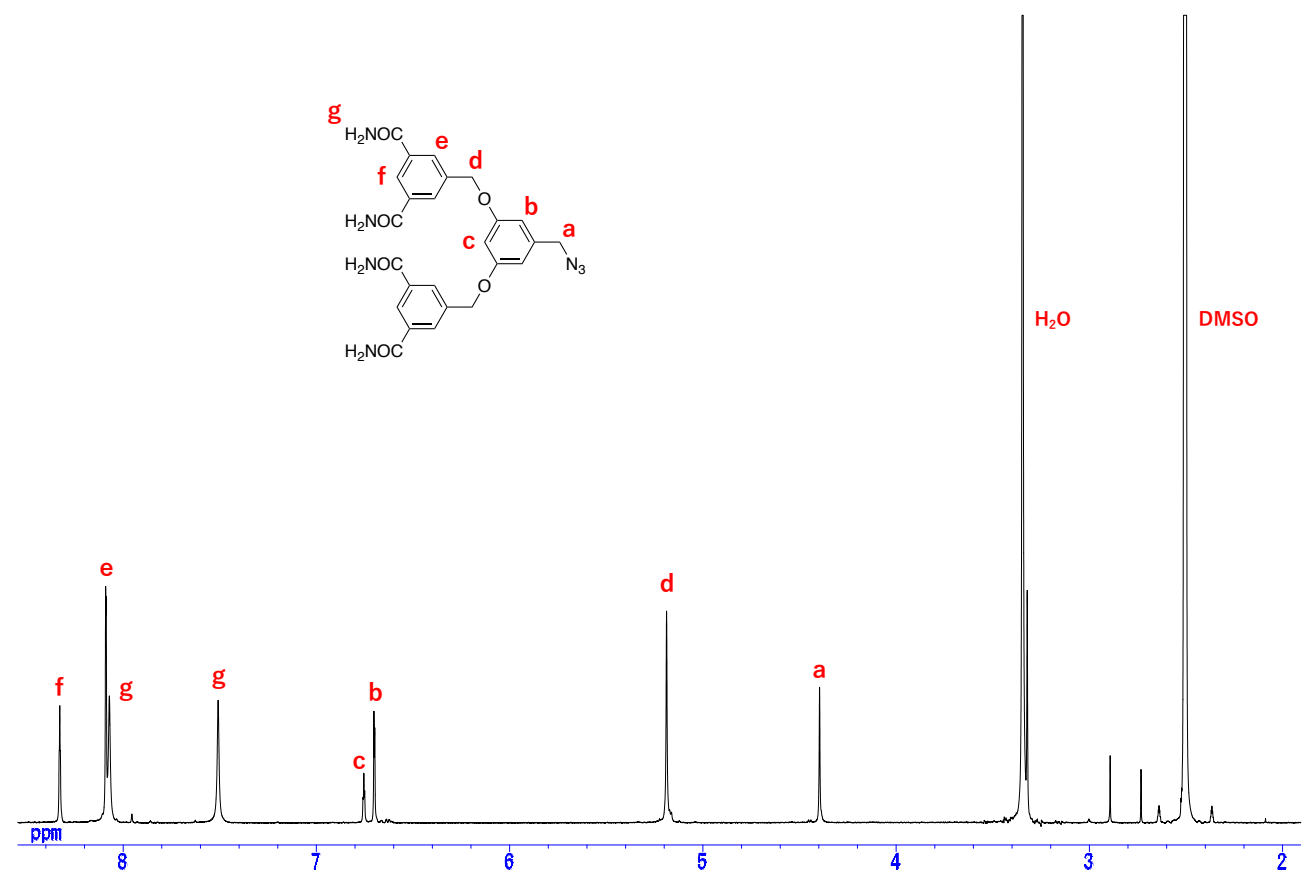

Figure S7. ${ }^{1} \mathrm{H}$ NMR spectrum of $\left(\mathbf{H}_{2} \mathbf{N O C}\right)_{4}-[\mathbf{G}-2]-\mathbf{N}_{3}(500 \mathrm{MHz}, \text { DMSO-d })_{6}$.

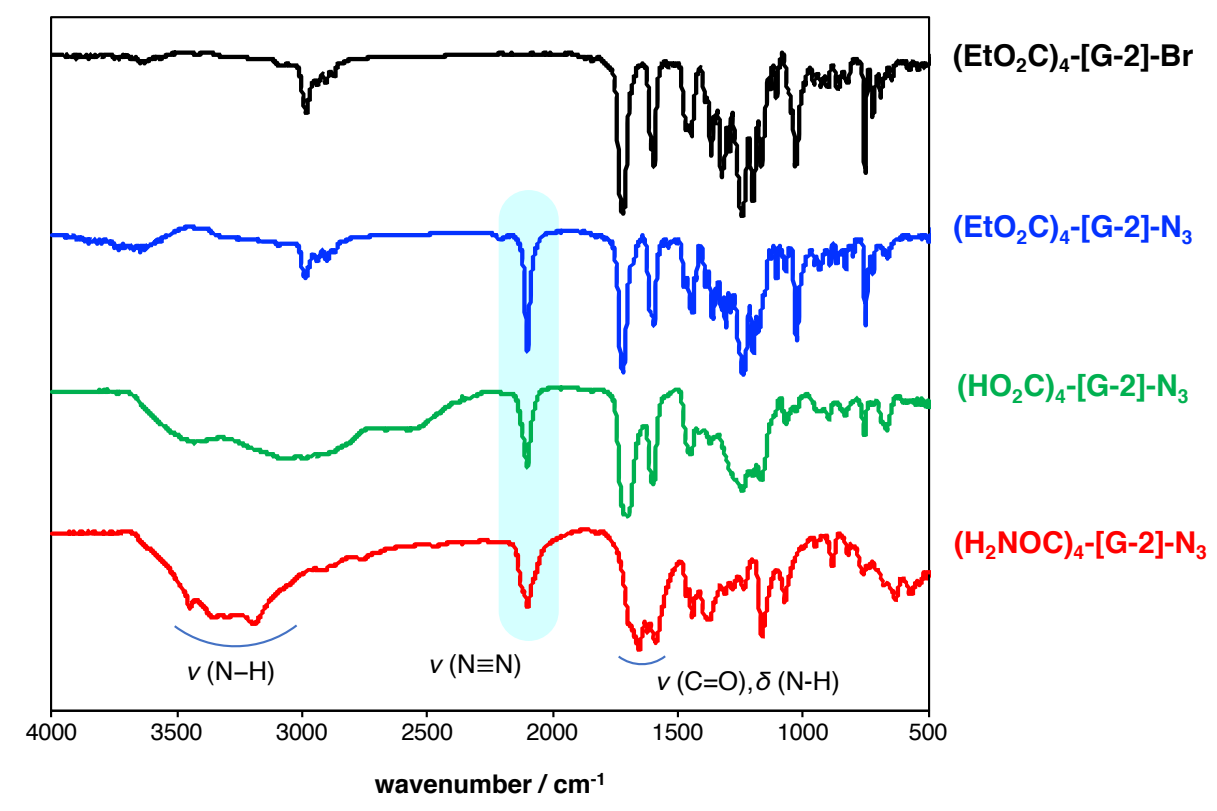

Figure S8. IR spectra of $(\mathbf{R})_{4}-[\mathbf{G}-2]-\mathbf{X}(\mathrm{KBr})$. 


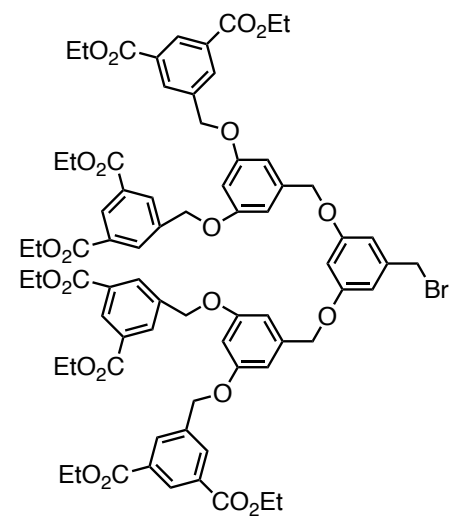

$\left(\mathrm{EtO}_{2} \mathrm{C}\right)_{8}-[\mathrm{G}-3]-\mathrm{Br}$

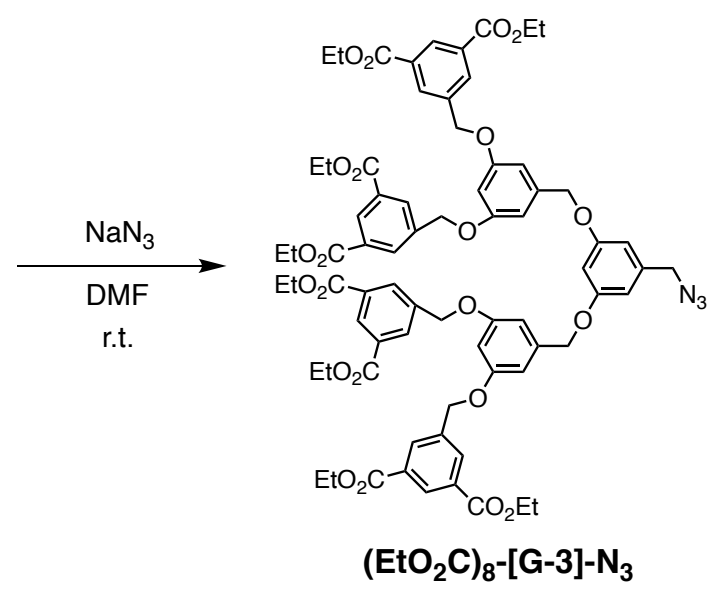

(EtO $\left._{2} \mathbf{C}\right)_{8}$-[G-3]-Br (1.50 g, $\left.1.08 \mathrm{mmol}\right)$ and sodium azide $(0.355 \mathrm{~g}, 5.47 \mathrm{mmol})$ in anhydrous DMF $(25 \mathrm{~mL})$ were stirred at r.t. for 1 day. The reaction mixture was precipitated into water. Following filtration, the product was dried in vacuo to afford $\left(\mathbf{E t O}_{2} \mathbf{C}\right)_{\mathbf{8}}-[\mathbf{G}-3]-\mathbf{N}_{3}$ as a white solid $(1.42 \mathrm{~g}, 98 \%)$.

${ }^{1} \mathrm{H}$ NMR (500 MHz, $\left.\mathrm{CDCl}_{3}\right): \delta / \mathrm{ppm} 8.64$ (t, $J=1.5 \mathrm{~Hz}, 4 \mathrm{H}$, aromatic), 8.30 (d, $J=1.5 \mathrm{~Hz}, 8 \mathrm{H}$, aromatic), $6.72(\mathrm{~d}, J=2.2 \mathrm{~Hz}, 4 \mathrm{H}$, aromatic), $6.61(\mathrm{t}, J=2.2 \mathrm{~Hz}, 2 \mathrm{H}$, aromatic), $6.58(\mathrm{t}, J=2.1 \mathrm{~Hz}, 1 \mathrm{H}$, aromatic), 6.55 (d, $J=2.2 \mathrm{~Hz}, 2 \mathrm{H}$, aromatic), $5.13\left(\mathrm{~s}, 8 \mathrm{H}, \mathrm{CH}_{2}\right), 5.01\left(\mathrm{~s}, 4 \mathrm{H}, \mathrm{CH}_{2}\right), 4.41$ (q, J=7.1 Hz, 16H, $\left.\mathrm{CH}_{2}\right), 4.27(\mathrm{~s}$,

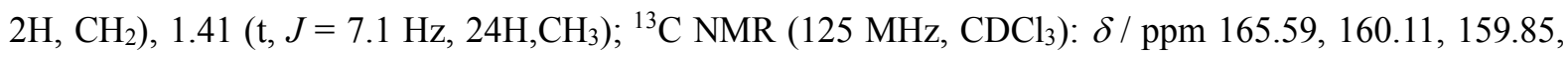
$139.45,137.76,137.70,132.57,131.36,130.24,107.27,106.55,101.83,101.70,69.90,69.14,61.48,54.79$, 14.34; FT-IR (KBr, cm $\left.{ }^{-1}\right):$ 2984, 2101, 1722, 1596, 1445, 1368, 1323, 1242, 1199, 1156, 1029, 825, 754. 


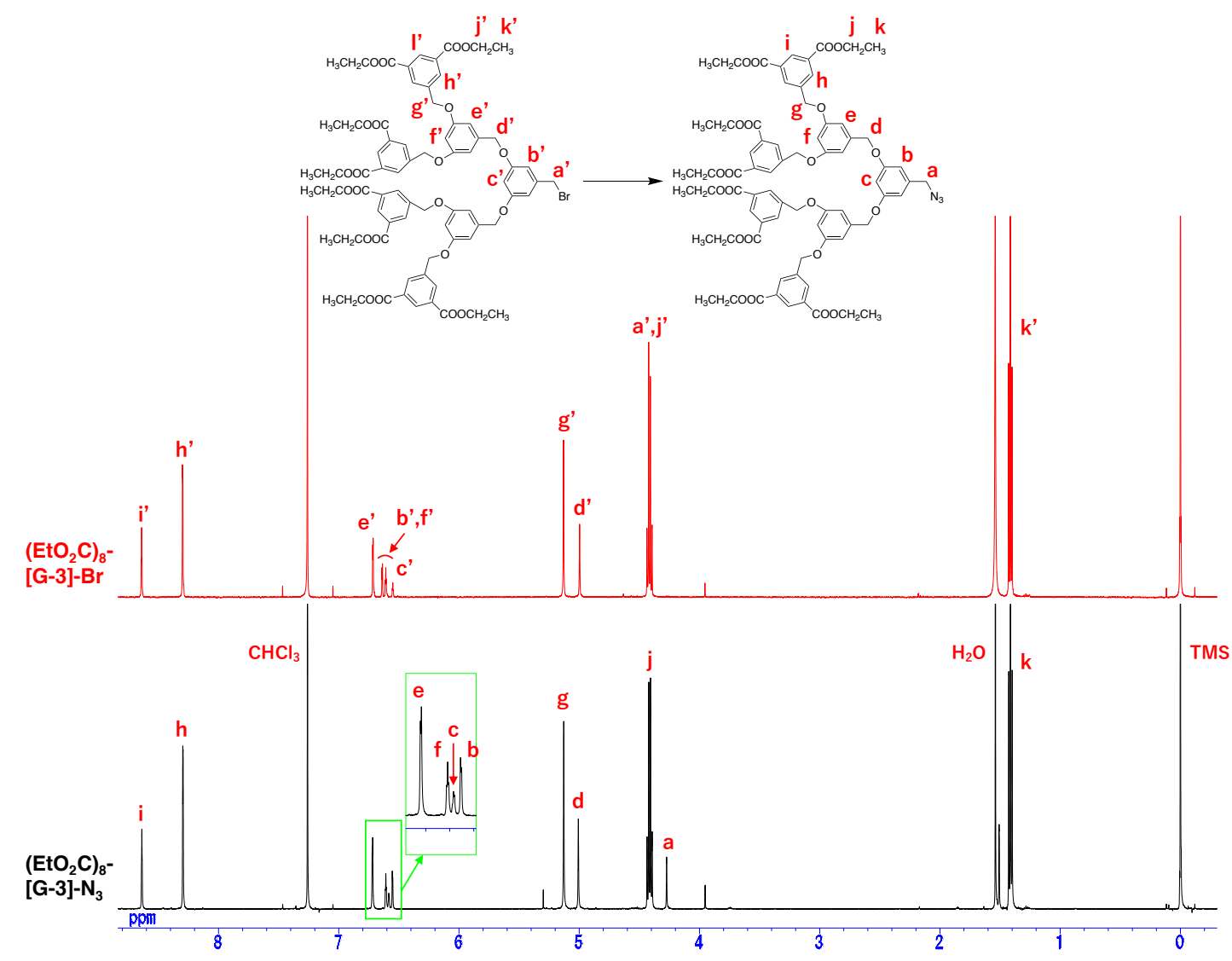

Figure S9. ${ }^{1} \mathrm{H}$ NMR spectra of $\left(\mathbf{E t O}_{2} \mathbf{C}\right)_{8}-[\mathbf{G}-3]-\mathbf{N}_{3}$ (black) and $\left(\mathbf{E t O}_{2} \mathbf{C}\right)_{8}-[\mathbf{G}-3]-\mathbf{B r}$ (red) $\left(500 \mathrm{MHz}, \mathrm{CDCl}_{3}\right)$.

\section{$\left(\mathrm{HO}_{2} \mathrm{C}\right)_{8-[\mathrm{G}-3]-\mathrm{N}_{3}}$}

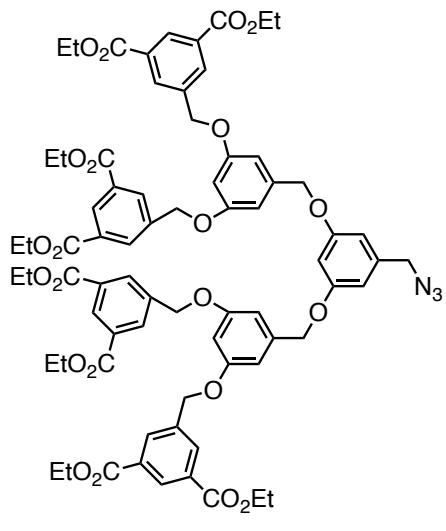

$\left(\mathrm{EtO}_{2} \mathrm{C}\right)_{8}-[\mathrm{G}-3]-\mathrm{N}_{3}$

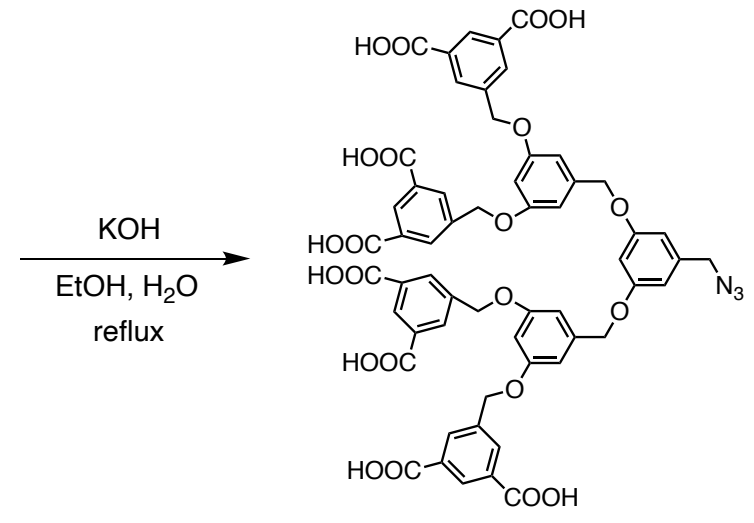

$(\mathrm{HOOC})_{8}-[\mathrm{G}-3]-\mathrm{N}_{3}$

$\left.\mathbf{( E t O}_{2} \mathbf{C}\right)_{\mathbf{8}}$-[G-3]-N $\mathbf{N}_{\mathbf{3}}(1.20 \mathrm{~g}, 0.894 \mathrm{mmol})$ and potassium hydroxide $(1.03 \mathrm{~g}, 18.4 \mathrm{mmol})$ were combined with EtOH $(20 \mathrm{~mL})$ and water $(15 \mathrm{~mL})$ in a round-bottom flask equipped with a reflux condenser. The mixture was refluxed for $6 \mathrm{~h}$. After evaporation, the resulting solution was neutralized with $1 \mathrm{M} \mathrm{HCl}$, at which point a white precipitate formed. Following filtration, the product was dried in vacuo at $40{ }^{\circ} \mathrm{C}$ to afford $\left(\mathbf{H O}_{2} \mathbf{C}\right)_{8^{-}}$ [G-3]-N 3 as a white solid (0.985 $\mathrm{g}, 98 \%)$.

${ }^{1} \mathrm{H}$ NMR (500 MHz, DMSO- $d_{6}$ ): $\delta / \mathrm{ppm} 8.42$ (s, 4H, aromatic), 8.23 (d, $J=1.3 \mathrm{~Hz}, 8 \mathrm{H}$, aromatic), 6.78 (d, 
$J=2.0 \mathrm{~Hz}, 4 \mathrm{H}$, aromatic), $6.74(\mathrm{t}, J=2.0 \mathrm{~Hz}, 2 \mathrm{H}$, aromatic), $6.68(\mathrm{t}, J=2.0 \mathrm{~Hz}, 1 \mathrm{H}$, aromatic), $6.63(\mathrm{~d}, J=$ $2.0 \mathrm{~Hz}, 2 \mathrm{H}$, aromatic), 5.27 (s, 8H, $\left.\mathrm{CH}_{2}\right), 5.04\left(\mathrm{~s}, 4 \mathrm{H}, \mathrm{CH}_{2}\right), 4.36\left(\mathrm{~s}, 2 \mathrm{H}, \mathrm{CH}_{2}\right) ;{ }^{13} \mathrm{C}$ NMR (125 MHz, DMSO$\left.d_{6}\right): \delta / \mathrm{ppm} 166.93,160.04,159.81,139.96,138.87,138.27,132.57,131.99,129.84,107.84,107.20,101.79$, 101.63, 69.60, 68.73, 54.01 ; FT-IR (KBr, cm $\left.{ }^{-1}\right): 3081,2103,1700,1598,1449,1372,1164,1057,900,757$, 668.

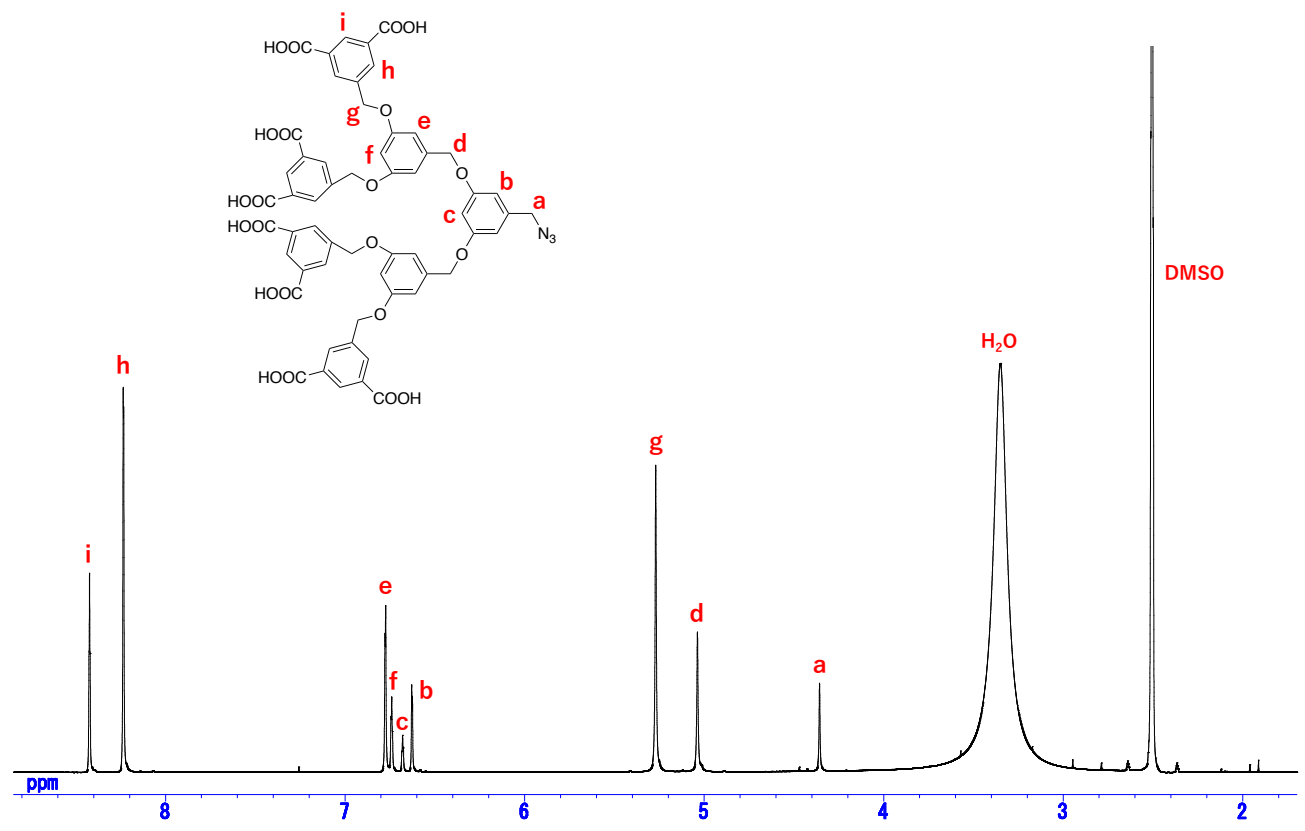

Figure S10. ${ }^{1} \mathrm{H}$ NMR spectrum of (HOOC) ${ }_{8}-[\mathbf{G}-3]-\mathbf{N}_{3}\left(500 \mathrm{MHz}, \mathrm{DMSO}-d_{6}\right)$.

\section{$\left(\mathrm{H}_{2} \mathrm{NOC}\right)_{8}-[\mathrm{G}-3]-\mathrm{N}_{3}$}
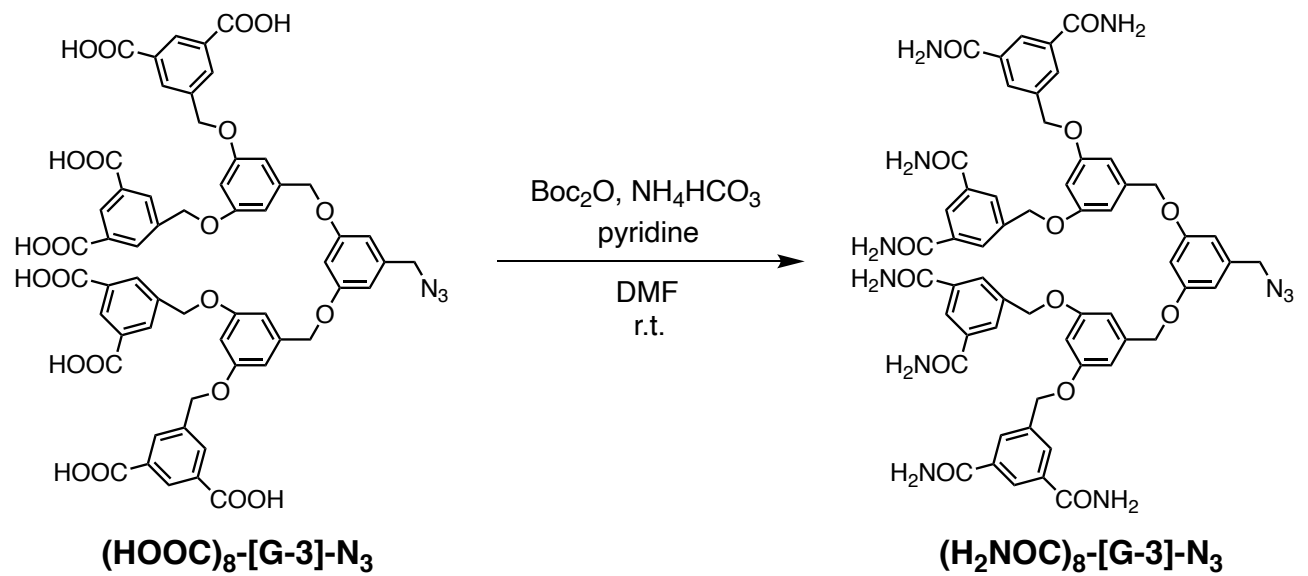

To a solution of $\left(\mathbf{H O}_{2} \mathbf{C}\right){ }_{8}$-[G-3]- $\mathbf{N}_{3}(0.629 \mathrm{~g}, 0.560 \mathrm{mmol})$, pyridine $(0.181 \mathrm{~mL}, 2.24 \mathrm{mmol})$, and di-tertbutyl dicarbonate $(2.01 \mathrm{~g}, 9.21 \mathrm{mmol})$ in anhydrous DMF $(20 \mathrm{~mL})$ was added ammonium bicarbonate $(0.710$ $\mathrm{g}, 8.98 \mathrm{mmol}$ ) and the mixture was allowed to stir at r.t. for 1 day. The resultant mixture was poured into water. Following filtration, the product was dried in vacuo at $40{ }^{\circ} \mathrm{C}$ overnight to afford $\left(\mathbf{H}_{2} \mathbf{N O C}\right)_{8}$-[G-3]- $\mathbf{N}_{3}$ as a white solid $(0.619 \mathrm{~g}, 99 \%)$. 
${ }^{1} \mathrm{H}$ NMR (500 MHz, DMSO- $d_{6}$ ): $\delta /$ ppm 8.33 (s, 4H, aromatic), 8.09 (s, 8H, aromatic), 8.06 (s, 8H, NH), $7.47(\mathrm{~s}, 8 \mathrm{H}, \mathrm{NH}), 6.78$ (s, 4H, aromatic), 6.73 (s, 2H, aromatic), 6.68 (s, 1H, aromatic), 6.65 (s, 2H, aromatic), 5.19 (s, 8H, $\left.\mathrm{CH}_{2}\right), 5.05$ (s, 4H, $\left.\mathrm{CH}_{2}\right), 4.38\left(\mathrm{~s}, 2 \mathrm{H}, \mathrm{CH}_{2}\right){ }^{13} \mathrm{C}$ NMR (100 MHz, DMSO- $\left.d_{6}\right): \delta / \mathrm{ppm} \mathrm{167.32,}$ $159.52,159.36,139.35,137.79,137.28,134.58,129.32$, 126.10, 107.31, 106.59, 101.28, 101.15, 69.12, 68.83, 53.51; FT-IR $\left(\mathrm{KBr}, \mathrm{cm}^{-1}\right)$ : 3360, 2103, 1668, 1591, 1446, 1374, 1167, 1052, 895, 828, 759, 627.

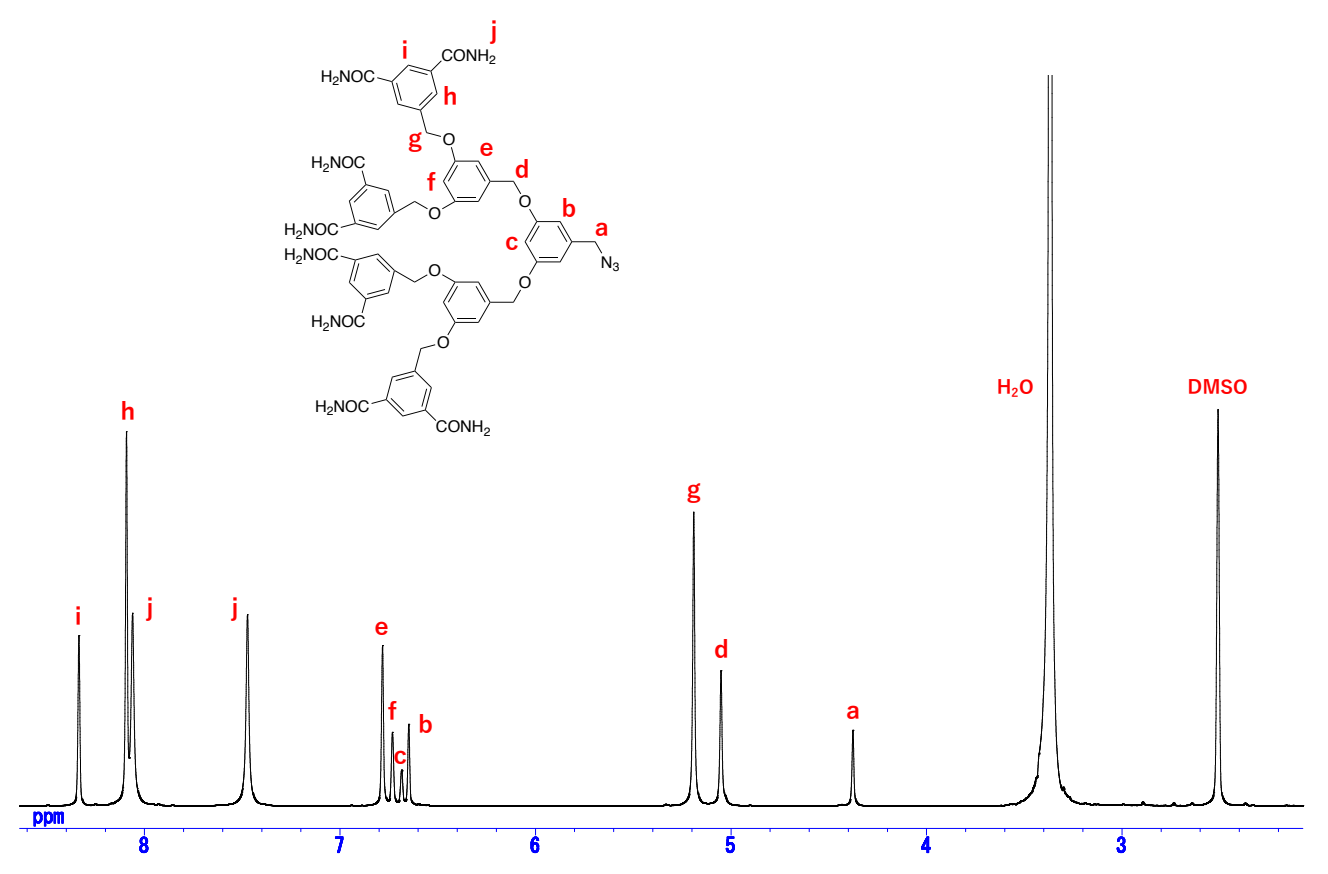

Figure S11. ${ }^{1} \mathrm{H}$ NMR spectrum of $\left(\mathbf{H}_{2} \mathbf{N O C}\right)_{8}-[\mathbf{G}-3]-\mathbf{N}_{3}\left(500 \mathrm{MHz}, \mathrm{DMSO}-d_{6}\right)$.

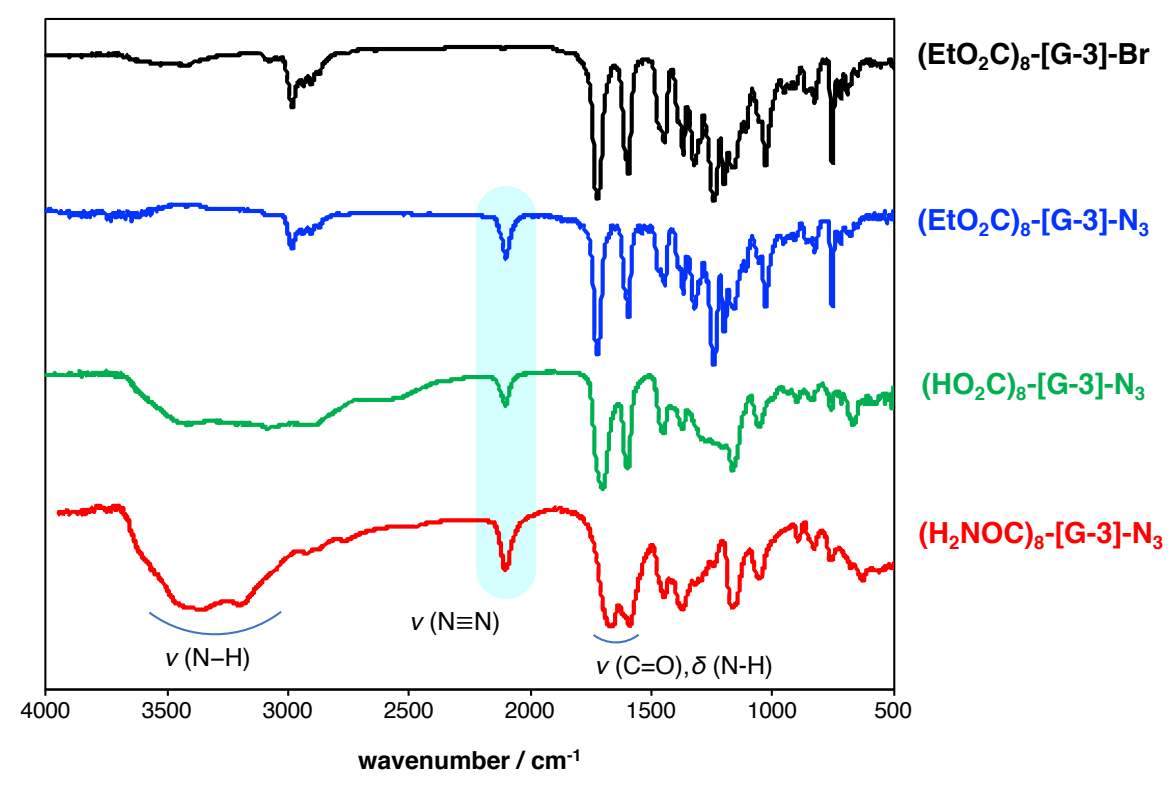

Figure S12. IR spectra of $(\mathbf{R})_{8}-[\mathbf{G}-3]-\mathbf{X}(\mathrm{KBr})$. 


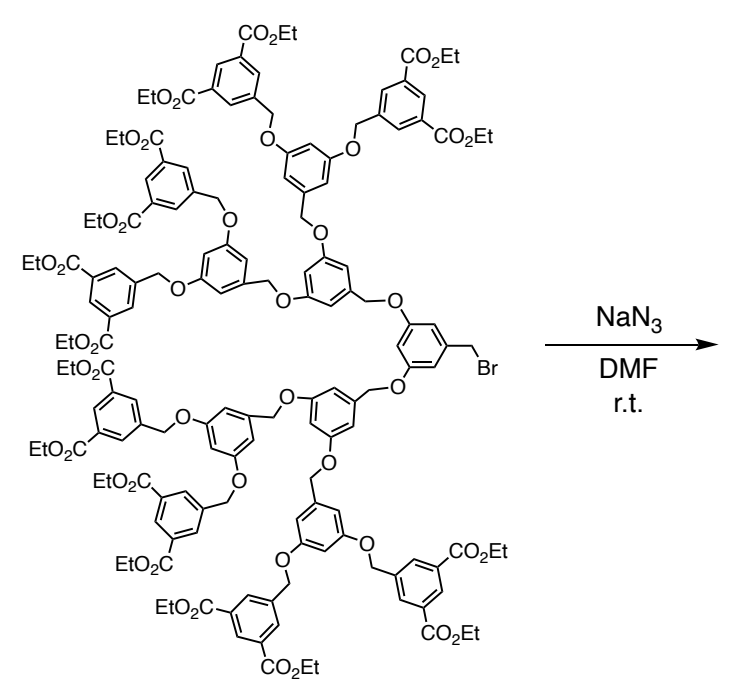

$\left(\mathrm{EtO}_{2} \mathrm{C}\right)_{16}-[\mathrm{G}-4]-\mathrm{Br}$

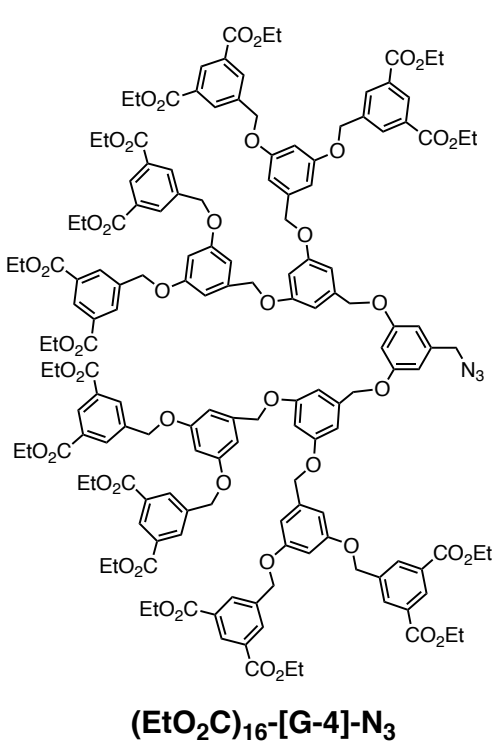

$\left(\mathrm{EtO}_{2} \mathrm{C}\right)_{16}-[\mathrm{G}-4]-\mathrm{N}_{3}$

$\left(\text { EtO }_{2} \mathbf{C}\right)_{16}$-[G-4]-Br $(1.50 \mathrm{~g}, 0.534 \mathrm{mmol})$ and sodium azide $(0.349 \mathrm{~g}, 5.37 \mathrm{mmol})$ in anhydrous DMF (30 $\mathrm{mL}$ ) were stirred at r.t. for 1 day. The reaction mixture was precipitated into water. Following filtration, the product was dried in vacuo to afford $\left(\mathbf{E t O}_{\mathbf{2}} \mathbf{C}\right)_{\mathbf{1 6}}-[\mathbf{G}-4]-\mathbf{N}_{3}$ as a white solid (1.50 g, quant.).

${ }^{1} \mathrm{H} \mathrm{NMR}\left(500 \mathrm{MHz}, \mathrm{CDCl}_{3}\right.$ ): $\delta / \mathrm{ppm} 8.62$ (t, $J=1.5 \mathrm{~Hz}, 8 \mathrm{H}$, aromatic), 8.28 (d, $J=1.4 \mathrm{~Hz}, 16 \mathrm{H}$, aromatic), $6.72(\mathrm{~d}, J=2.1 \mathrm{~Hz}, 8 \mathrm{H}$, aromatic), $6.68(\mathrm{~d}, J=2.1 \mathrm{~Hz}, 4 \mathrm{H}$, aromatic), 6.59 (t, $J=2.2 \mathrm{~Hz}, 5 \mathrm{H}$, aromatic), $6.57\left(\mathrm{t}, J=2.0 \mathrm{~Hz}, 2 \mathrm{H}\right.$, aromatic), 6.54 (d, $J=2.1 \mathrm{~Hz}, 2 \mathrm{H}$, aromatic), $5.11\left(\mathrm{~s}, 16 \mathrm{H}, \mathrm{CH}_{2}\right), 4.99$ (s, 8H, $\left.\mathrm{CH}_{2}\right)$, $4.98\left(\mathrm{~s}, 4 \mathrm{H}, \mathrm{CH}_{2}\right), 4.40$ (q, $J=7.1 \mathrm{~Hz}, 32 \mathrm{H}$, aromatic), $4.24\left(\mathrm{~s}, 2 \mathrm{H}, \mathrm{CH}_{2}\right), 1.40\left(\mathrm{t}, J=7.1 \mathrm{~Hz}, 48 \mathrm{H}, \mathrm{CH}_{3}\right) ;{ }^{13} \mathrm{C}$ NMR (125 MHz, $\left.\mathrm{CDCl}_{3}\right): \delta /$ ppm 165.56, 162.52, 160.17, 160.05, 159.82, 139.54, 139.16, 137.70, 132.55, $131.33,130.21,107.27,106.58,106.43,101.81,101.63,101.55,70.03,69.87,69.10,61.47,54.78,14.34$; FT-IR (KBr, cm $\left.{ }^{-1}\right): 3424,2981,2101,1721,1596,1446,1368,1324,1241,1198,1160,1029,833,754,718$. 


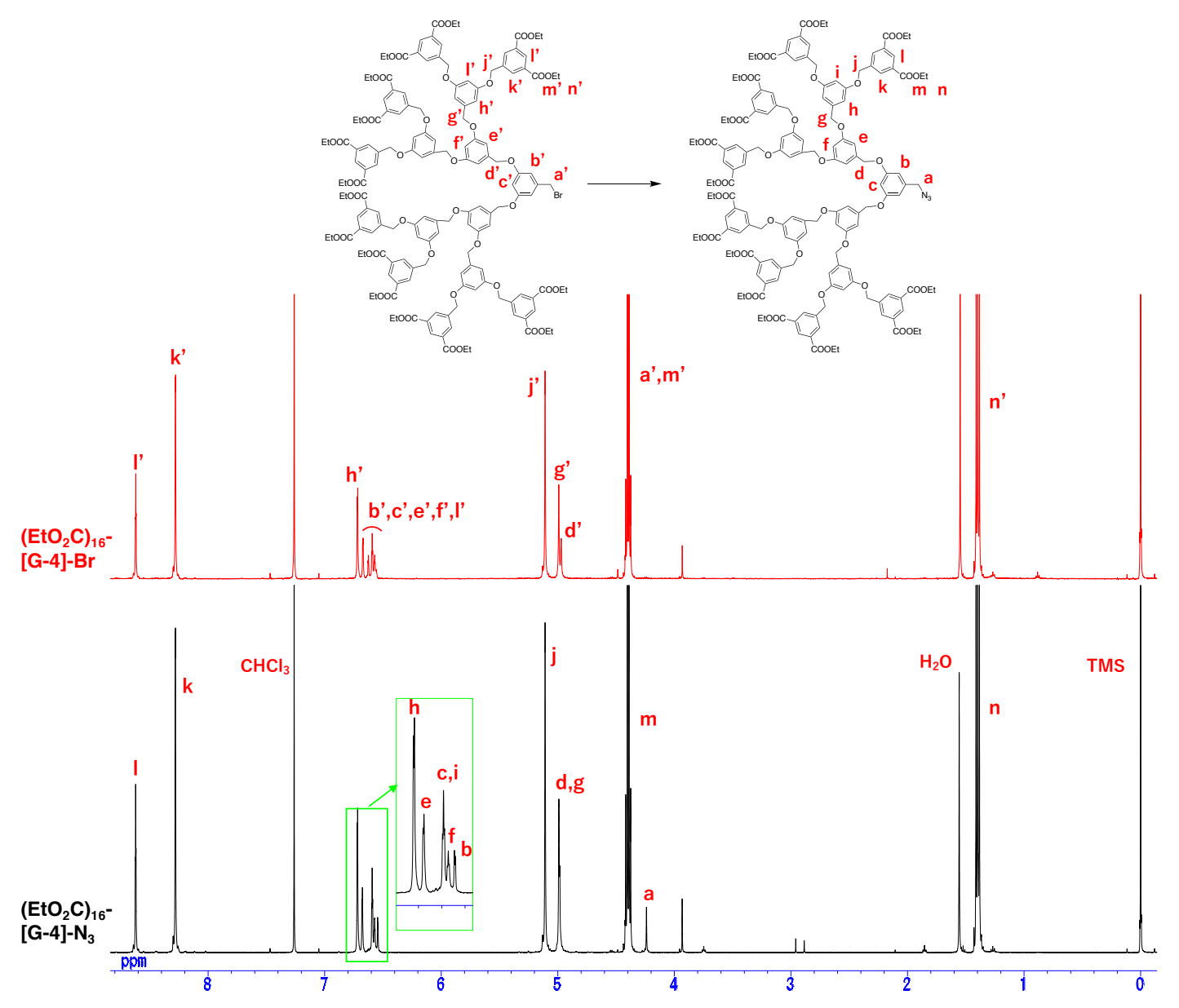

Figure S13. ${ }^{1} \mathrm{H}$ NMR spectra of $\left(\mathbf{E t O}_{2} \mathbf{C}\right)_{16}-[\mathbf{G}-4]-\mathbf{N}_{3}$ (black) and $\left(\mathbf{E t O}_{2} \mathbf{C}\right)_{16}-[\mathbf{G}-4]-\mathbf{B r}$ (red) $(500 \mathrm{MHz}$, $\mathrm{CDCl}_{3}$ ). 

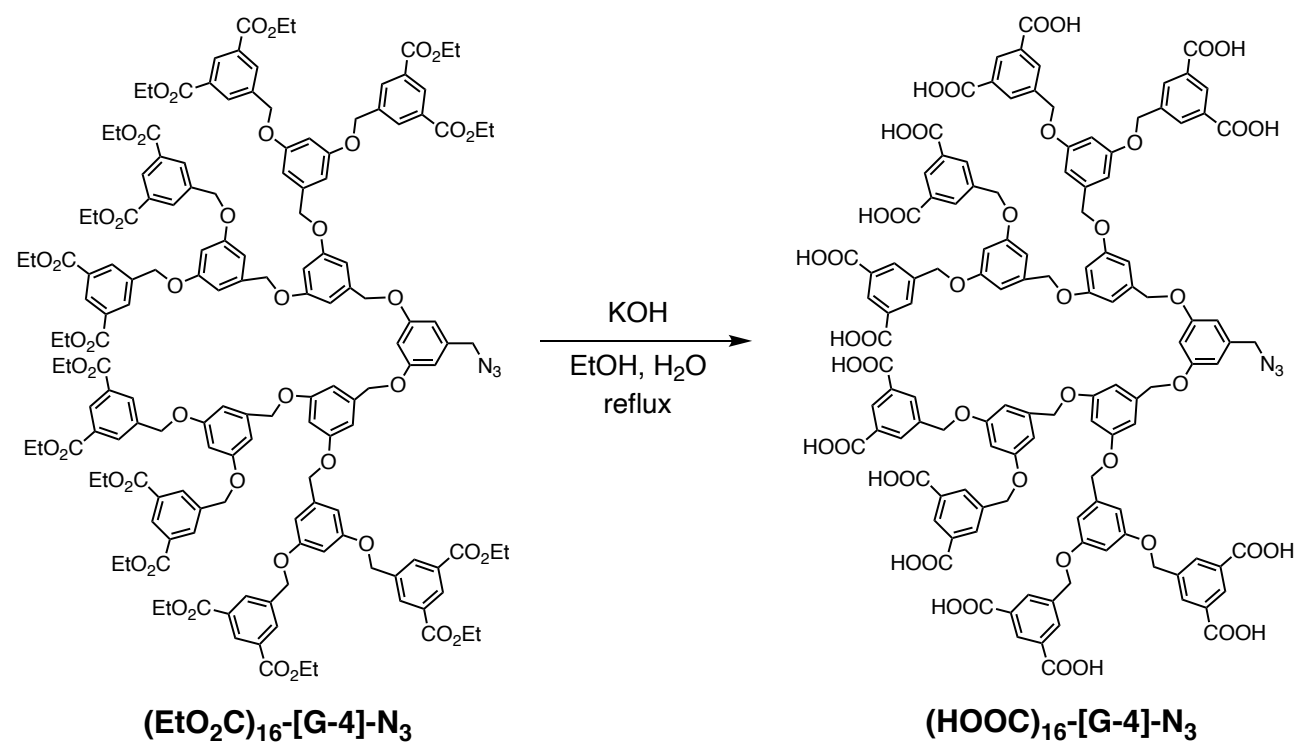

$\left(\mathbf{E t O}_{\mathbf{2}} \mathbf{C}\right)_{16}$-[G-4]-N $\mathbf{3}(0.771 \mathrm{~g}, 0.278 \mathrm{mmol})$ and potassium hydroxide $(0.690 \mathrm{~g}, 12.3 \mathrm{mmol})$ were combined with EtOH $(10 \mathrm{~mL})$ and water $(10 \mathrm{~mL})$ in a round-bottom flask equipped with a reflux condenser. The mixture was refluxed for $1.5 \mathrm{~h}$. After evaporation, the resulting solution was neutralized with $1 \mathrm{M} \mathrm{HCl}$, at which point a suspension formed. The product was collected by centrifugation, washed with water, and dried in vacuo at $40{ }^{\circ} \mathrm{C}$ to afford $\left(\mathbf{H O}_{\mathbf{2}} \mathbf{C}\right)_{\mathbf{1 6}}$-[G-4]- $\mathbf{N}_{\mathbf{3}}$ as a brown solid $(0.985 \mathrm{~g}, 98 \%)$.

${ }^{1} \mathrm{H}$ NMR (500 MHz, DMSO- $d_{6}$ ): $\delta /$ ppm 8.42 (s, 8H, aromatic), 8.22 (s, 16H, aromatic), 6.77 (s, 8H, aromatic), 6.73 (s, 4H, aromatic), 6.71 (s, 4H, aromatic), 6.69 (s, 1H, aromatic), 6.65 (s, 2H, aromatic), 6.62 (s, $2 \mathrm{H}$, aromatic), $5.25\left(\mathrm{~s}, 16 \mathrm{H}, \mathrm{CH}_{2}\right), 5.02\left(\mathrm{~s}, 12 \mathrm{H}, \mathrm{CH}_{2}\right), 4.32\left(\mathrm{~s}, 2 \mathrm{H}, \mathrm{CH}_{2}\right) . ;{ }^{13} \mathrm{C}$ NMR (125 MHz, DMSO$\left.d_{6}\right): \delta / \operatorname{ppm} 166.91,160.09,159.95,159.79,140.01,138.84,132.59,131.91,129.84,107.21,107.02,101.57$, 69.60, 68.72, 54.00; FT-IR $\left(\mathrm{KBr}, \mathrm{cm}^{-1}\right)$ : 3087, 2103, 1703, 1596, 1449, 1372, 1160, 1052, 833, 758, 673. 


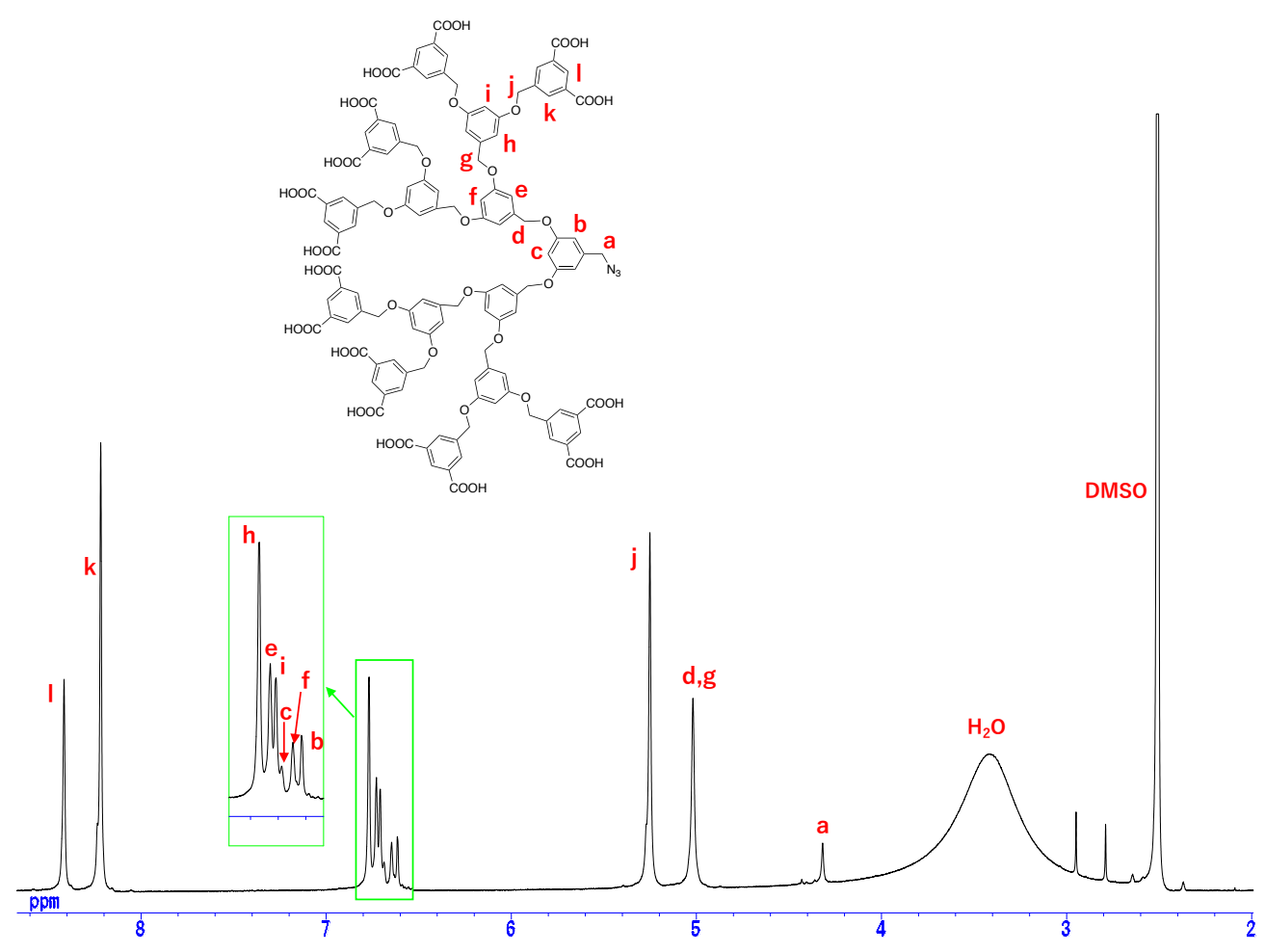

Figure S14. ${ }^{1} \mathrm{H}$ NMR spectrum of (HOOC) $)_{16}-[\mathbf{G}-4]-N_{3}\left(500 \mathrm{MHz}, \mathrm{DMSO}-d_{6}\right)$.

\section{$\left(\mathrm{H}_{2} \mathrm{NOC}\right)_{16-[\mathrm{G}-4]-\mathrm{N}_{3}}$}
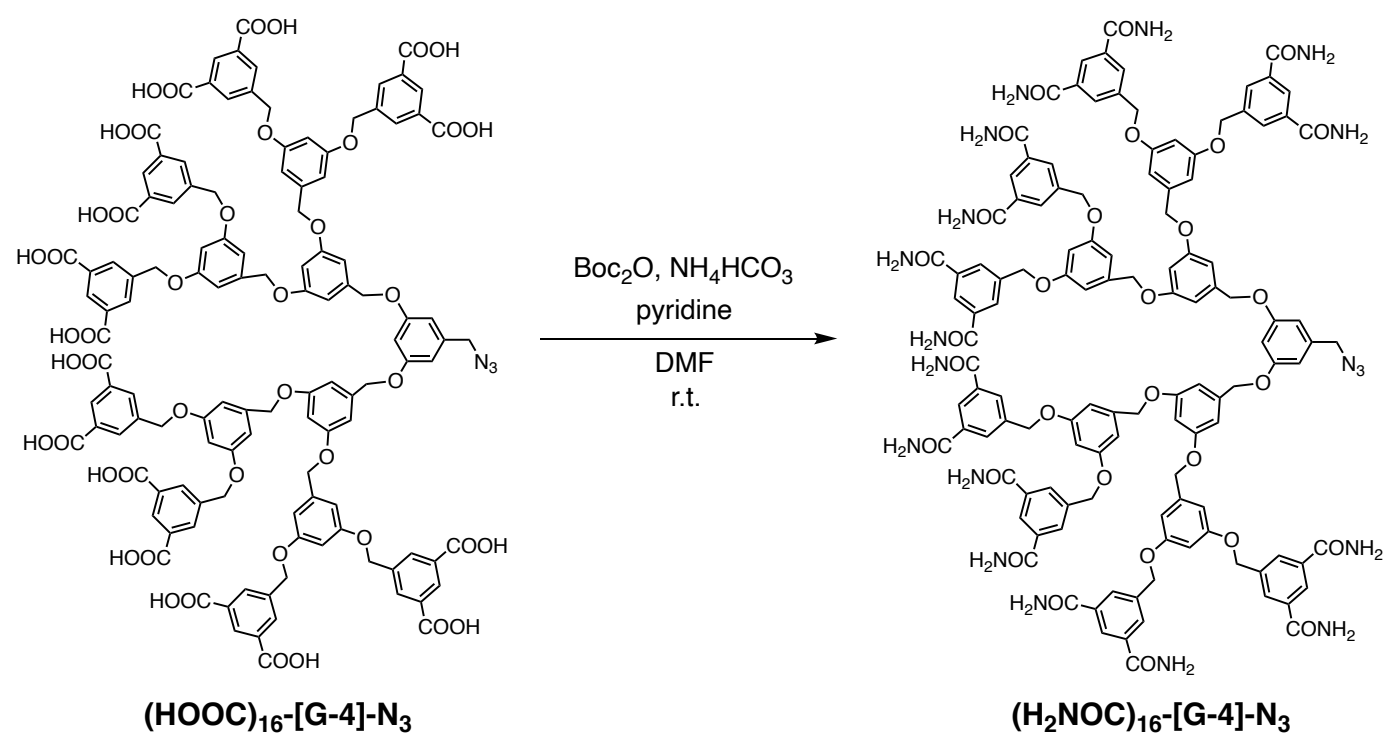

To a solution of $\left(\mathbf{H O}_{2} \mathbf{C}\right)_{16}-[\mathbf{G}-4]-\mathbf{N}_{3}(0.635 \mathrm{~g}, 0.273 \mathrm{mmol})$, pyridine $(0.176 \mathrm{~mL}, 2.19 \mathrm{mmol})$, and di-tertbutyl dicarbonate $(2.05 \mathrm{~g}, 9.39 \mathrm{mmol})$ in anhydrous DMF $(15 \mathrm{~mL})$ was added ammonium bicarbonate $(0.744$ $\mathrm{g}, 9.41 \mathrm{mmol}$ ) and the mixture was allowed to stir at r.t. for 1 day. The resultant mixture was poured into water. The product was collected by centrifugation from the resulting suspension, washed with water, and dried in vacuo at $40{ }^{\circ} \mathrm{C}$ to afford $\left(\mathbf{H}_{2} \mathbf{N O C}\right)_{16-[G-4]-N_{3}}$ as a pale brown solid $(0.621 \mathrm{~g}, 98 \%)$. 
${ }^{1} \mathrm{H}$ NMR (500 MHz, DMSO- $d_{6}$ ): $\delta /$ ppm 8.33 (s, 8H, aromatic), 8.09 (d, $J=1.2 \mathrm{~Hz}, 16 \mathrm{H}$, aromatic), 8.07 (s, 16H, NH), $7.49(\mathrm{~s}, 16 \mathrm{H}, \mathrm{NH}), 6.78(\mathrm{~d}, J=1.9 \mathrm{~Hz}, 8 \mathrm{H}$, aromatic), 6.74-6.72 (m, 9H, aromatic), 6.66 (s, $2 \mathrm{H}$, aromatic), $6.64\left(\mathrm{~d}, J=1.8 \mathrm{~Hz}, 2 \mathrm{H}\right.$, aromatic), $5.18\left(\mathrm{~s}, 16 \mathrm{H}, \mathrm{CH}_{2}\right), 5.04\left(\mathrm{~s}, 12 \mathrm{H}, \mathrm{CH}_{2}\right), 4.34\left(\mathrm{~s}, 2 \mathrm{H}, \mathrm{CH}_{2}\right)$; ${ }^{13} \mathrm{C}$ NMR (125 MHz, DMSO- $\left.d_{6}\right): \delta /$ ppm 167.87, 162.81, 160.14, 160.00, 159.91, 139.95, 139.72, 137.81, 135.15, 129.90, 126.67, 107.16, 101.61, 69.65, 69.34, 54.01; FT-IR $\left(\mathrm{KBr}, \mathrm{cm}^{-1}\right): 3357,3204,2102,1664$, $1592,1446,1375,1155,1055,493$.

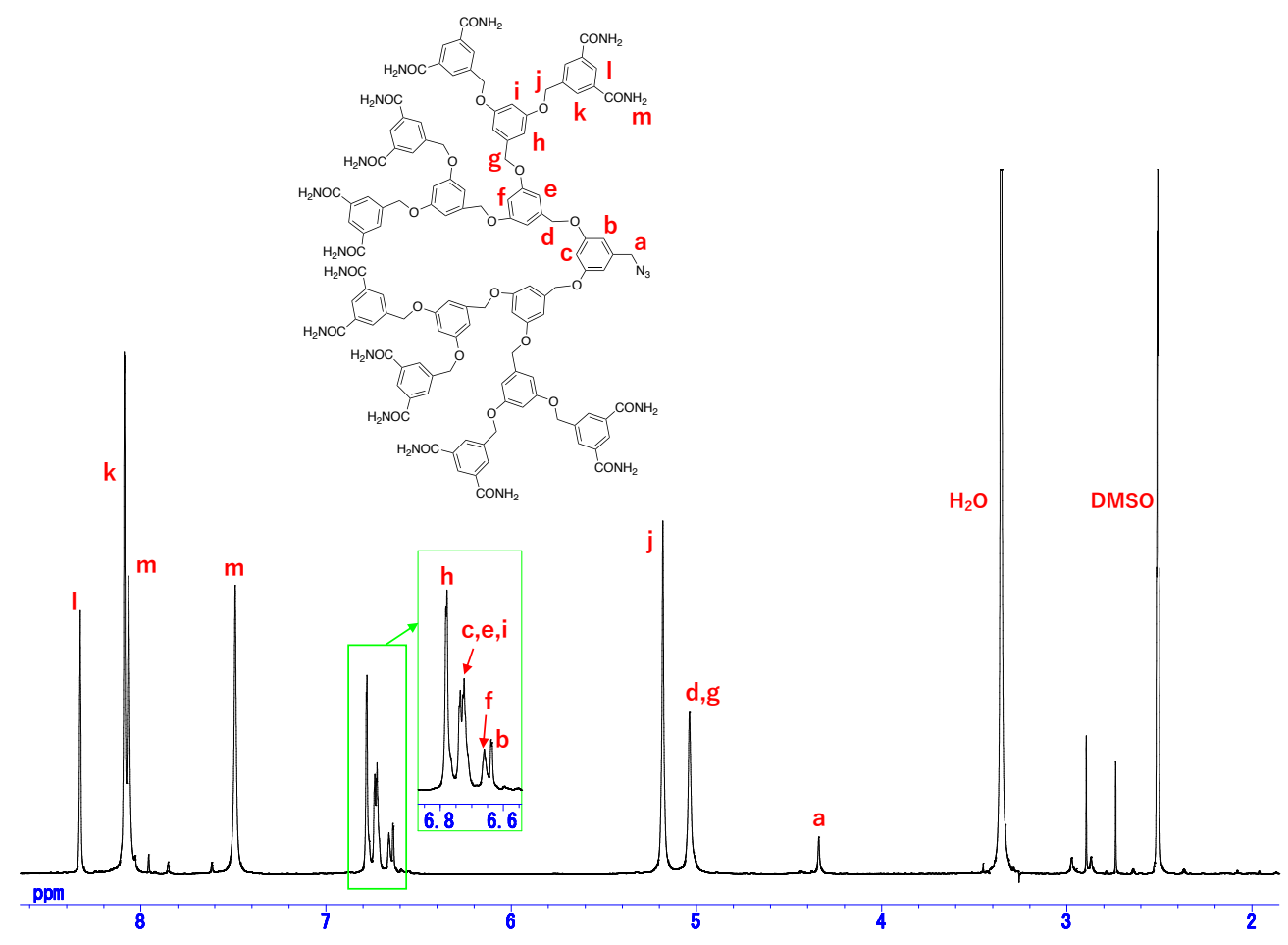

Figure S15. ${ }^{1} \mathrm{H}$ NMR spectrum of $\left(\mathbf{H}_{\mathbf{2}} \mathbf{N O C}\right)_{16}-[\mathbf{G}-4]-\mathbf{N}_{\mathbf{3}}\left(500 \mathrm{MHz}, \mathrm{DMSO}-d_{6}\right)$.

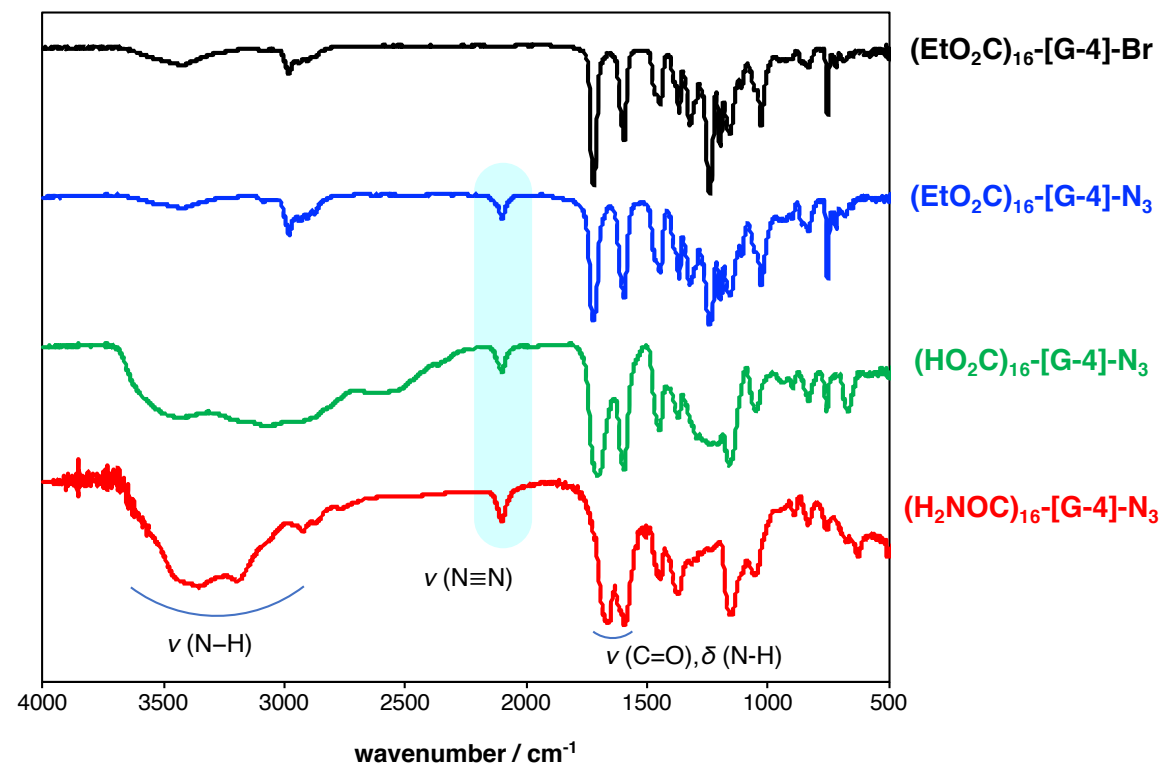

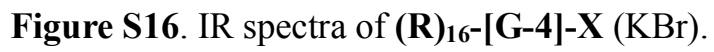




\subsection{Synthesis of $M D_{\text {ester }}$}

\section{MDester[G-1]}
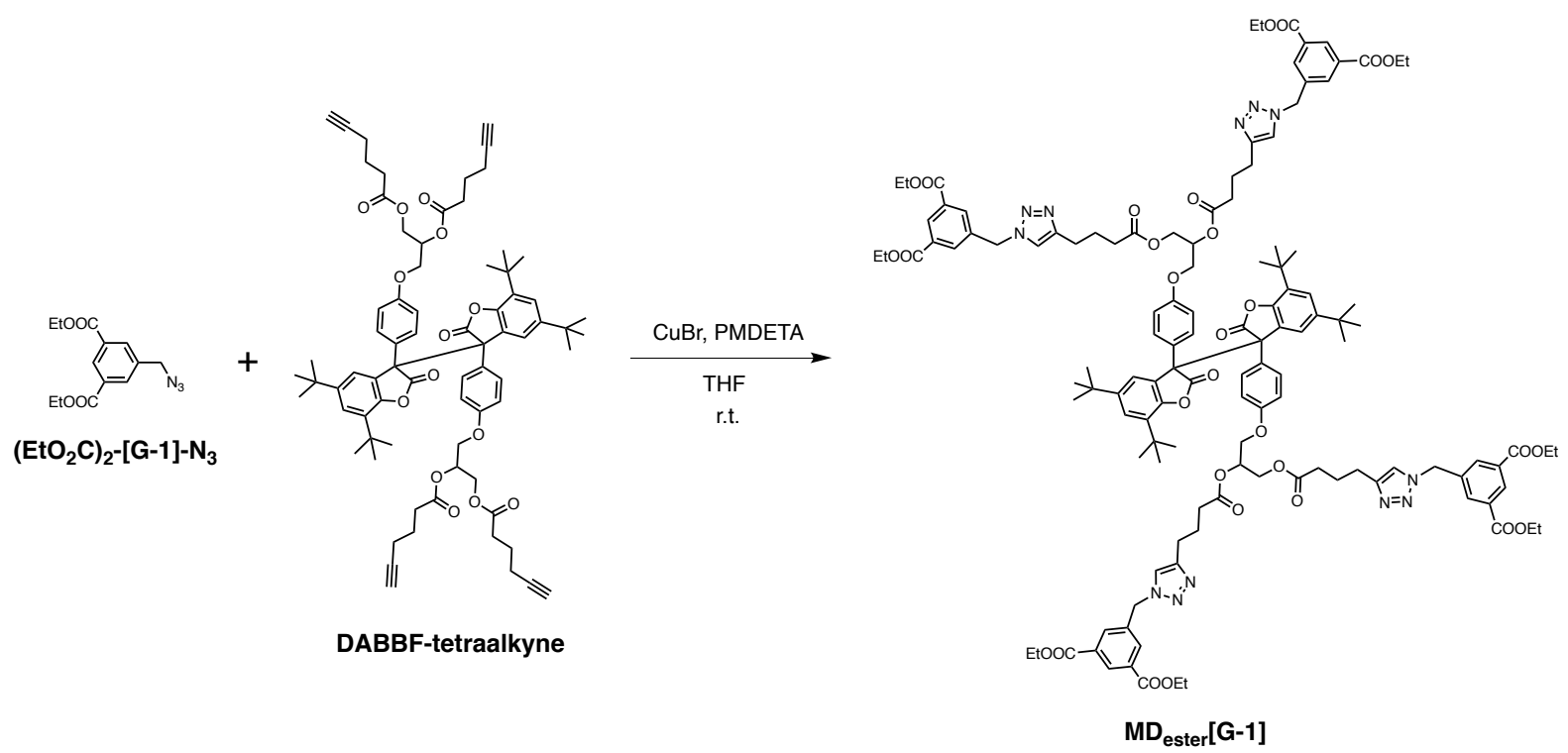

Under a nitrogen atmosphere, PMDETA $(0.584 \mathrm{~mL}, 2.80 \mathrm{mmol})$ was added to a solution of $\quad\left(\mathbf{E t O}_{2} \mathbf{C}\right)_{2}$-[G1]-N $\mathbf{3}(0.388 \mathrm{~g}, 1.40 \mathrm{mmol})$, DABBF-tetraalkyne (0.280 g, $0.234 \mathrm{~mol}), \mathrm{CuBr}(0.203 \mathrm{~g}, 1.41 \mathrm{mmol})$, and $\mathrm{Cu}$ $(88.7 \mathrm{mg}, 1.40 \mathrm{mmol})$ in anhydrous THF $(10 \mathrm{~mL})$ bubbled with nitrogen for 20 minutes. After being bubbled with nitrogen for another 20 minutes, the resulting solution was allowed to stir at r.t. for $1 \mathrm{~h}$. The reaction mixture was diluted with THF and filtered through a column filled with neutral alumina in order to remove the copper complex. Following evaporation, the crude product was subsequently purified by flash chromatography (dichloromethane/ethyl acetate gradient, $0-100 \%$ ethyl acetate) and freeze-drying using benzene as a solvent to give the product as a white solid $(0.441 \mathrm{~g}, 82 \%)$.

${ }^{1} \mathrm{H}$ NMR (500 MHz, $\mathrm{CDCl}_{3}$ ): $\delta / \mathrm{ppm} 8.63$ (s, 4H, aromatic), 8.13 (s, 8H, aromatic), 7.36 (m, 4H, aromatic), 7.30 (br, $4 \mathrm{H}$, aromatic), 6.80 (d, $J=5.7 \mathrm{~Hz}, 4 \mathrm{H}$, aromatic), 5.59 (s, $\left.8 \mathrm{H}, \mathrm{CH}_{2}\right), 5.38$ (m, 2H, CH), 4.47-4.24 (m, 4H, CH $)_{2}, 4.39$ (q, $\left.J=7.1 \mathrm{~Hz}, 16 \mathrm{H}, \mathrm{CH}_{2}\right), 4.11\left(\mathrm{~m}, 4 \mathrm{H}, \mathrm{CH}_{2}\right), 2.74$ (q, $\left.J=7.2 \mathrm{~Hz}, 8 \mathrm{H}, \mathrm{CH}_{2}\right), 2.40$ (m, $\left.8 \mathrm{H}, \mathrm{CH}_{2}\right), 1.99$ (m, 8H, $\left.\mathrm{CH}_{2}\right), 1.39$ (t, $\left.J=7.1 \mathrm{~Hz}, 24 \mathrm{H}, \mathrm{CH}_{3}\right), 1.30$ (br, $\left.\mathrm{H}, \mathrm{CH}_{3}\right), 1.17$ (br, $\left.\mathrm{H}_{,} \mathrm{CH}_{3}\right) ;{ }^{13} \mathrm{C}$ NMR (125 MHz, $\mathrm{CDCl}_{3}$ ): $\delta / \mathrm{ppm} 172.80,172.48,165.15,158.32,158.31,149.10,147.77,147.73,135.89,133.59$, 133.12 , 132.37, 131.89, 130.76, 128.33, 124.16, 121.10, 121.07, 113.27, 69.53, 66.05, 62.48, 61.63, 53.19, $34.72,34.36,33.47,33.28,31.53,29.66,24.87,24.84,24.45,24.42,14.29$; FT-IR $\left(\mathrm{KBr}, \mathrm{cm}^{-1}\right): 2961,1793$, 1726, 1607, 1509, 1458, 1367, 1320, 1243, 1197, 1132, 1090, 1026, 821, 753; MALDI-TOF: $m / z$ [M+Na] calcd: 2330.0 , found: 2332.3 . 

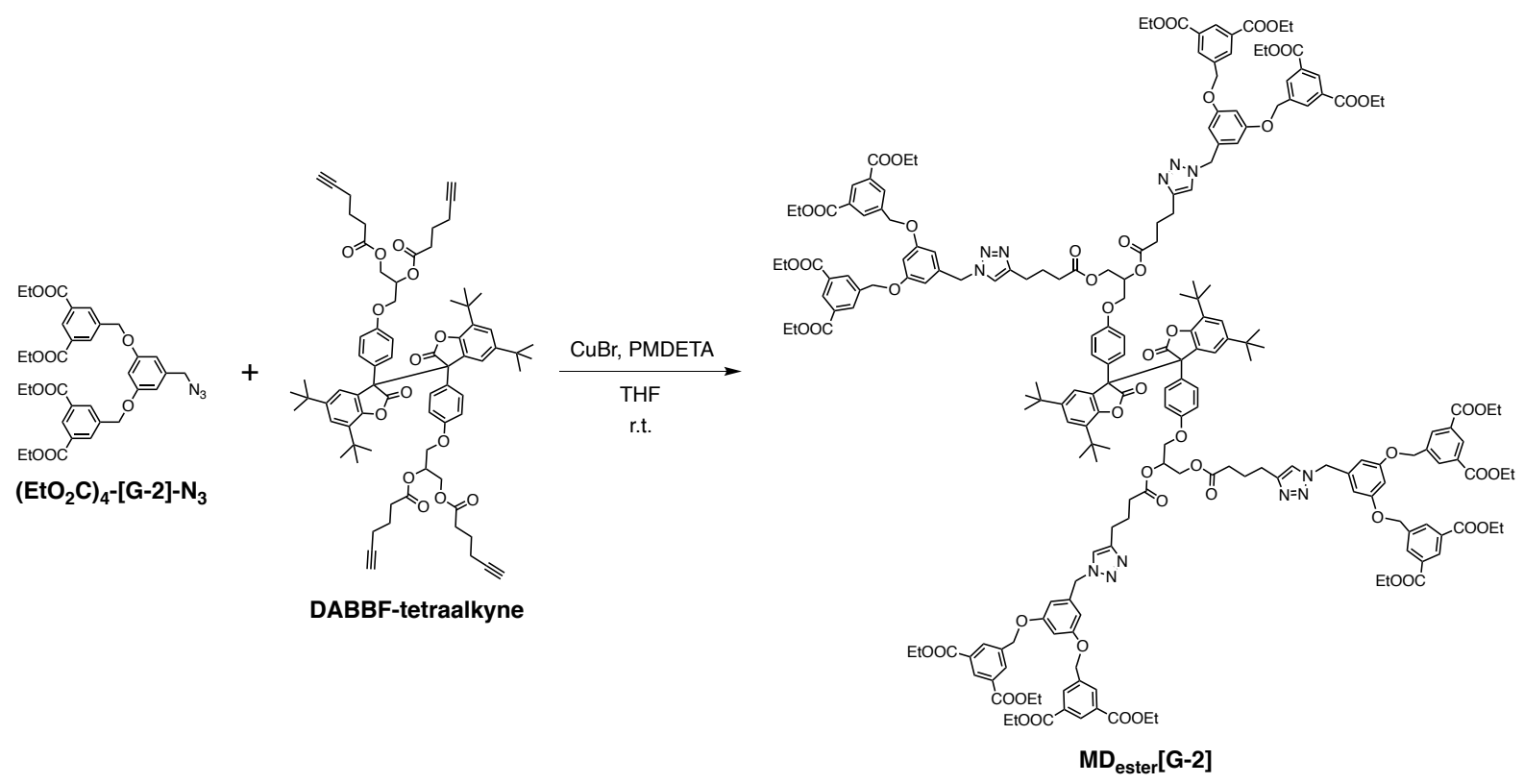

Under a nitrogen atmosphere, PMDETA $(0.395 \mathrm{~mL}, 1.89 \mathrm{mmol})$ was added to a solution of $\left.\mathbf{( E t O}_{\mathbf{2}} \mathbf{C}\right)_{4}$-[G2]-N 3 (0.602 g, $0.949 \mathrm{mmol})$, DABBF-tetraalkyne (0.192 g, $0.160 \mathrm{~mol}), \mathrm{CuBr}(0.135 \mathrm{~g}, 0.939 \mathrm{mmol})$, and $\mathrm{Cu}(60.5 \mathrm{mg}, 0.952 \mathrm{mmol})$ in anhydrous THF $(10 \mathrm{~mL})$ bubbled with nitrogen for 20 minutes. After being bubbled with nitrogen for another 20 minutes, the resulting solution was allowed to stir at r.t. for $1 \mathrm{~h}$. The reaction mixture was diluted with THF and filtered through a column filled with neutral alumina in order to remove the copper complex. Following evaporation, the crude product was subsequently purified by flash chromatography (dichloromethane/ethyl acetate gradient, $0-100 \%$ ethyl acetate) and freeze-drying using benzene as a solvent to give the product as a white solid $(0.445 \mathrm{~g}, 74 \%)$.

${ }^{1} \mathrm{H}$ NMR (500 MHz, $\mathrm{CDCl}_{3}$ ): $\delta / \mathrm{ppm} 8.62$ (s, 8H, aromatic), 8.25 (d, $J=0.9 \mathrm{~Hz}, 16 \mathrm{H}$, aromatic), 7.32 (m, $4 \mathrm{H}$, aromatic), 7.28 (br, $4 \mathrm{H}$, aromatic), 6.79 (d, $J=6.6 \mathrm{~Hz}, 4 \mathrm{H}$, aromatic), 6.58 (t, $J=2.0 \mathrm{~Hz}, 4 \mathrm{H}$, aromatic), $6.53\left(\mathrm{~m}, 8 \mathrm{H}\right.$, aromatic), $5.41\left(\mathrm{~s}, 8 \mathrm{H}, \mathrm{CH}_{2}\right), 5.37(\mathrm{~m}, 2 \mathrm{H}, \mathrm{CH}), 5.06\left(\mathrm{~s}, 16 \mathrm{H}_{1} \mathrm{CH}_{2}\right), 4.46-4.23\left(\mathrm{~m}, 4 \mathrm{H}, \mathrm{CH}_{2}\right)$, 4.40 (q, $\left.J=7.1 \mathrm{~Hz}, 32 \mathrm{H}, \mathrm{CH}_{2}\right), 4.09$ (m, 4H, CH$), 2.72$ (q, $\left.J=7.5 \mathrm{~Hz}, 8 \mathrm{H}, \mathrm{CH}_{2}\right), 2.40\left(\mathrm{~m}, 8 \mathrm{H}, \mathrm{CH}_{2}\right), 1.97$ $\left(\mathrm{m}, 8 \mathrm{H}, \mathrm{CH}_{2}\right), 1.40\left(\mathrm{t}, J=7.1 \mathrm{~Hz}, 48 \mathrm{H}, \mathrm{CH}_{3}\right), 1.29\left(\mathrm{br}, 18 \mathrm{H}, \mathrm{CH}_{3}\right), 1.15\left(\mathrm{br}, 18 \mathrm{H}, \mathrm{CH}_{3}\right) ;{ }^{13} \mathrm{C} \mathrm{NMR}(125 \mathrm{MHz}$, $\left.\mathrm{CDCl}_{3}\right): \delta / \mathrm{ppm} 172.83,172.52,165.54,160.03,158.31,149.09,147.57,147.53,137.49,137.39,133.57$, $132.57,131.35,130.27,128.34,121.11,121.08,113.26,107.37,101.96,69.50,69.11,66.04,62.48,61.51$, 53.90, 52.48, 34.74, 34.36, 33.52, 33.33, 31.54, 29.65, 24.92, 24.89, 24.48, 24.45, 14.34; FT-IR (KBr, cm$\left.{ }^{1}\right)$ : 2962, 1724, 1597, 1509, 1457, 1367, 1325, 1242, 1199, 1028, 755; MALDI-TOF: $m / z$ [M+Na] ${ }^{+}$calcd: 3754.5, found: 3755.7 . 

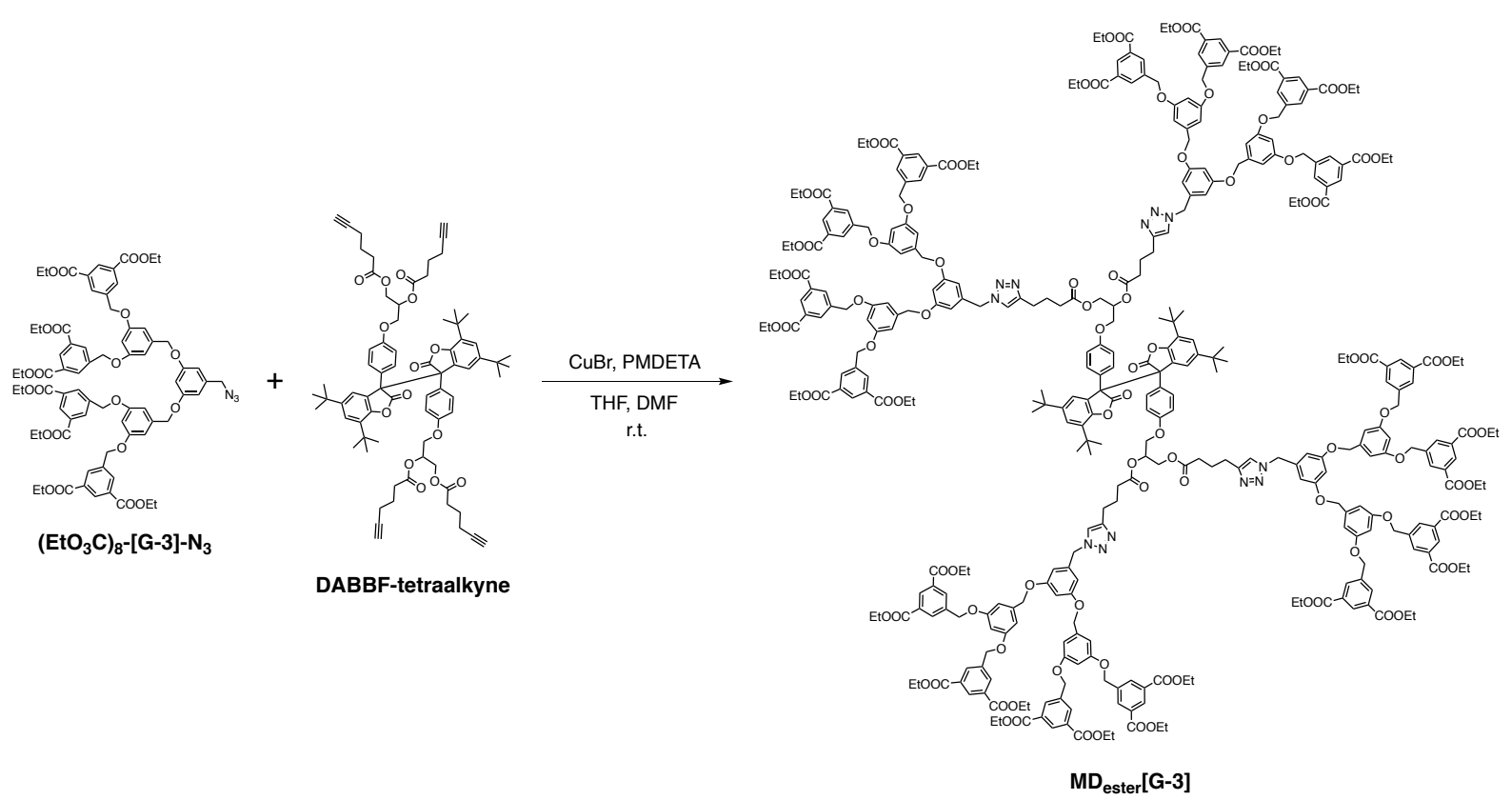

Under a nitrogen atmosphere, PMDETA $(0.217 \mathrm{~mL}, 1.04 \mathrm{mmol})$ was added to a solution of $\quad\left(\mathbf{E t O}_{2} \mathbf{C}\right)_{8}$-[G3]-N $3(0.701 \mathrm{~g}, 0.520 \mathrm{mmol})$, DABBF-tetraalkyne $(0.109 \mathrm{~g}, 91.0 \mu \mathrm{mol}), \mathrm{CuBr}(74.6 \mathrm{mg}, 0.520 \mathrm{mmol})$, and $\mathrm{Cu}(33.1 \mathrm{mg}, 0.521 \mathrm{mmol})$ in anhydrous THF $(15 \mathrm{~mL})$ and DMF $(10 \mathrm{~mL})$ bubbled with nitrogen for 20 minutes. After being bubbled with nitrogen for another 20 minutes, the resulting solution was allowed to stir at r.t. for $1 \mathrm{~h}$. The reaction mixture was diluted with THF and filtered through a column filled with neutral alumina in order to remove the copper complex. Following evaporation, the crude product was subsequently purified by flash chromatography (dichloromethane/ethyl acetate gradient, $0-40 \%$ ethyl acetate) and freezedrying using benzene as a solvent to give the product as a white solid $(0.366 \mathrm{~g}, 61 \%)$.

${ }^{1} \mathrm{H}$ NMR (500 MHz, $\mathrm{CDCl}_{3}$ ): $\delta / \mathrm{ppm} 8.61$ (s, 16H, aromatic), 8.26 (s, 32H, aromatic), 7.32 (d, $J=7.7 \mathrm{~Hz}$, $4 \mathrm{H}$, aromatic), 7.30-7.24 (br, 4H, aromatic), 6.78 (d, $J=7.8 \mathrm{~Hz}, 4 \mathrm{H}$, aromatic), 6.67 (d, $J=2.0 \mathrm{~Hz}, 16 \mathrm{H}$, aromatic), 6.58 (t, $J=1.9 \mathrm{~Hz}, 8 \mathrm{H}$, aromatic), 6.54 (m, 4H, aromatic), 6.48 (m, 8H, aromatic), 5.38 (s, 8H, $\left.\mathrm{CH}_{2}\right), 5.36$ (m, 2H, CH), 5.09 (s, 32H, $\left.\mathrm{CH}_{2}\right), 4.90$ (s, 16H, $\left.\mathrm{CH}_{2}\right), 4.44-4.21$ (m, 4H, $\left.\mathrm{CH}_{2}\right), 4.39$ (q, J = 7.1 $\mathrm{Hz}, 64 \mathrm{H}, \mathrm{CH}_{2}$ ), 4.08 (m, 4H, $\mathrm{CH}_{2}$ ), 2.68 (q, $\left.J=8.1 \mathrm{~Hz}, 8 \mathrm{H}, \mathrm{CH}_{2}\right), 2.38\left(\mathrm{~m}, 8 \mathrm{H}, \mathrm{CH}_{2}\right), 1.95\left(\mathrm{~m}, 8 \mathrm{H} \mathrm{CH}_{2}\right.$ ), 1.39 (t, $\left.J=7.1 \mathrm{~Hz}, 96 \mathrm{H}, \mathrm{CH}_{3}\right), 1.27$ (br, $\left.18 \mathrm{H}, \mathrm{CH}_{3}\right), 1.14$ (br, $\left.18 \mathrm{H}, \mathrm{CH}_{3}\right) ;{ }^{13} \mathrm{C} \mathrm{NMR} \mathrm{(125} \mathrm{MHz,} \mathrm{CDCl}_{3}$ ): $\delta /$ ppm 172.81, 172.49, 165.54, 160.20, 159.78, 158.29, 149.07, 147.52, 147.48, 139.21, 137.77, 137.67, 137.30, 133.53, 132.54, 131.29, 130.92, 130.18, 121.11, 113.27, 107.09, 106.54, 101.81, 101.62, 69.83, $69.48,69.05,66.02,62.45,61.46,60.40,53.91,52.44,34.68,34.33,33.51,33.31,31.51,29.63,24.92,24.90$, 24.50, 24.45, 14.33; FT-IR (KBr, cm $\left.{ }^{-1}\right): 2979,1722,1596,1509,1447,1368,1324,1241,1198,1160,1028$, 833, 755, 717; MALDI-TOF: $m / z$ [M+Na $]^{+}$calcd: 6603.5, found: 6605.7 . 

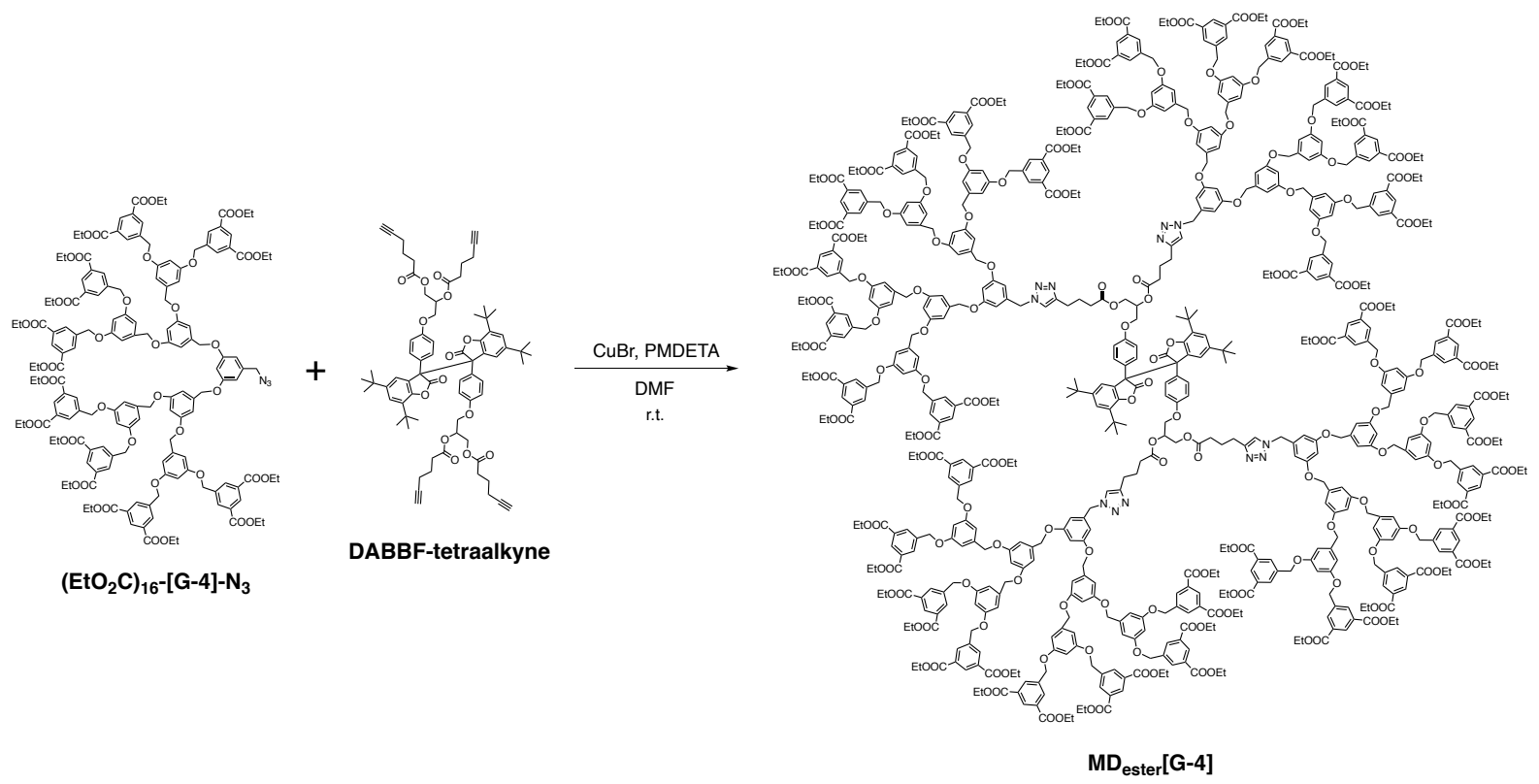

Under a nitrogen atmosphere, PMDETA $(0.158 \mathrm{~mL}, 0.757 \mathrm{mmol})$ was added to a solution of $\left(\mathbf{E t O}_{\mathbf{2}} \mathbf{C}\right)_{\mathbf{1 6}^{-}}$ [G-4]-N 3 (0.702 g, $0.253 \mathrm{mmol})$, DABBF-tetraalkyne (61.8 mg, $51.5 \mu \mathrm{mol}), \mathrm{CuBr}$ (64.8 mg, $0.452 \mathrm{mmol})$, and $\mathrm{Cu}(20.8 \mathrm{mg}, 0.327 \mathrm{mmol})$ in anhydrous DMF $(50 \mathrm{~mL})$ bubbled with nitrogen for 20 minutes. After being bubbled with nitrogen for another 20 minutes, the resulting solution was allowed to stir at r.t. for $3 \mathrm{~h}$. The reaction mixture was diluted with THF and filtered through a column filled with neutral alumina in order to remove the copper complex. Following evaporation, the crude product was subsequently purified by flash chromatography (dichloromethane/ethyl acetate gradient, 0-20\% ethyl acetate) and freeze-drying using benzene as a solvent to give the product as a white solid $(0.278 \mathrm{~g}, 44 \%)$.

${ }^{1} \mathrm{H}$ NMR (500 MHz, $\mathrm{CDCl}_{3}$ ): $\delta / \mathrm{ppm} 8.58$ (s, 32H, aromatic), 8.23 (s, 64H, aromatic), 7.32 (d, $J=8.1 \mathrm{~Hz}$, $4 \mathrm{H}$, aromatic), 7.25 (br, $4 \mathrm{H}$, aromatic), 6.77 (d, $J=6.7 \mathrm{~Hz}, 4 \mathrm{H}$, aromatic), 6.68 (d, $J=1.8 \mathrm{~Hz}, 32 \mathrm{H}$, aromatic), $6.62(\mathrm{~s}, 16 \mathrm{H}$, aromatic), 6.55 (s, 16H, aromatic), 6.52 (s, 12H, aromatic), 6.48 (s, 8H, aromatic), 5.35 (s, 9H, $\mathrm{CH}_{2}$ and $\mathrm{CH}$ ), $5.05\left(\mathrm{~s}, 64 \mathrm{H}, \mathrm{CH}_{2}\right), 4.92\left(\mathrm{~s}, 32 \mathrm{H}, \mathrm{CH}_{2}\right), 4.86\left(\mathrm{~s}, 16 \mathrm{H}, \mathrm{CH}_{2}\right), 4.42-4.20\left(\mathrm{~m}, 4 \mathrm{H}, \mathrm{CH}_{2}\right), 4.36$ (q, $\left.J=7.1 \mathrm{~Hz}, 128 \mathrm{H}, \mathrm{CH}_{2}\right), 4.06$ (br, 4H, $\left.\mathrm{CH}_{2}\right), 2.67$ (q, $\left.J=7.7 \mathrm{~Hz}, 8 \mathrm{H}, \mathrm{CH}_{2}\right), 2.36\left(\mathrm{~m}, 8 \mathrm{H}, \mathrm{CH}_{2}\right), 1.93(\mathrm{~m}, 8 \mathrm{H}$, $\left.\mathrm{CH}_{2}\right), 1.36\left(\mathrm{t}, J=7.1 \mathrm{~Hz}, 192 \mathrm{H}, \mathrm{CH}_{3}\right), 1.26$ (br, 18H, $\left.\mathrm{CH}_{3}\right), 1.12\left(\mathrm{br}, 18 \mathrm{H}, \mathrm{CH}_{3}\right) ;{ }^{13} \mathrm{C} \mathrm{NMR}(125 \mathrm{MHz}$, $\left.\mathrm{CDCl}_{3}\right): \delta /$ ppm 172.79, 172.46, 165.98, 165.51, 160.28, 159.99, 159.76, 147.51, 139.48, 138.93, 137.69, 137.32, 132.49, 131.26, 130.14, 128.34, 121.13, 113.35, 107.10, 106.57, 106.44, 101.54, 69.97, 69.80, 69.47, 69.01, 61.44, 53.87, 52.42, 34.32, 33.52, 33.31, 31.51, 29.63, 24.94, 24.47, 14.32; FT-IR $\left(\mathrm{KBr}, \mathrm{cm}^{-1}\right)$ : 2980, 1722, 1596, 1447, 1368, 1324, 1240, 1198, 1160, 1029, 929, 833, 755, 718, 682. ; MALDI-TOF: $\mathrm{m} / \mathrm{z}$ $[\mathrm{M}+\mathrm{Na}]^{+}$calcd: 12302 , found: 12303 . 


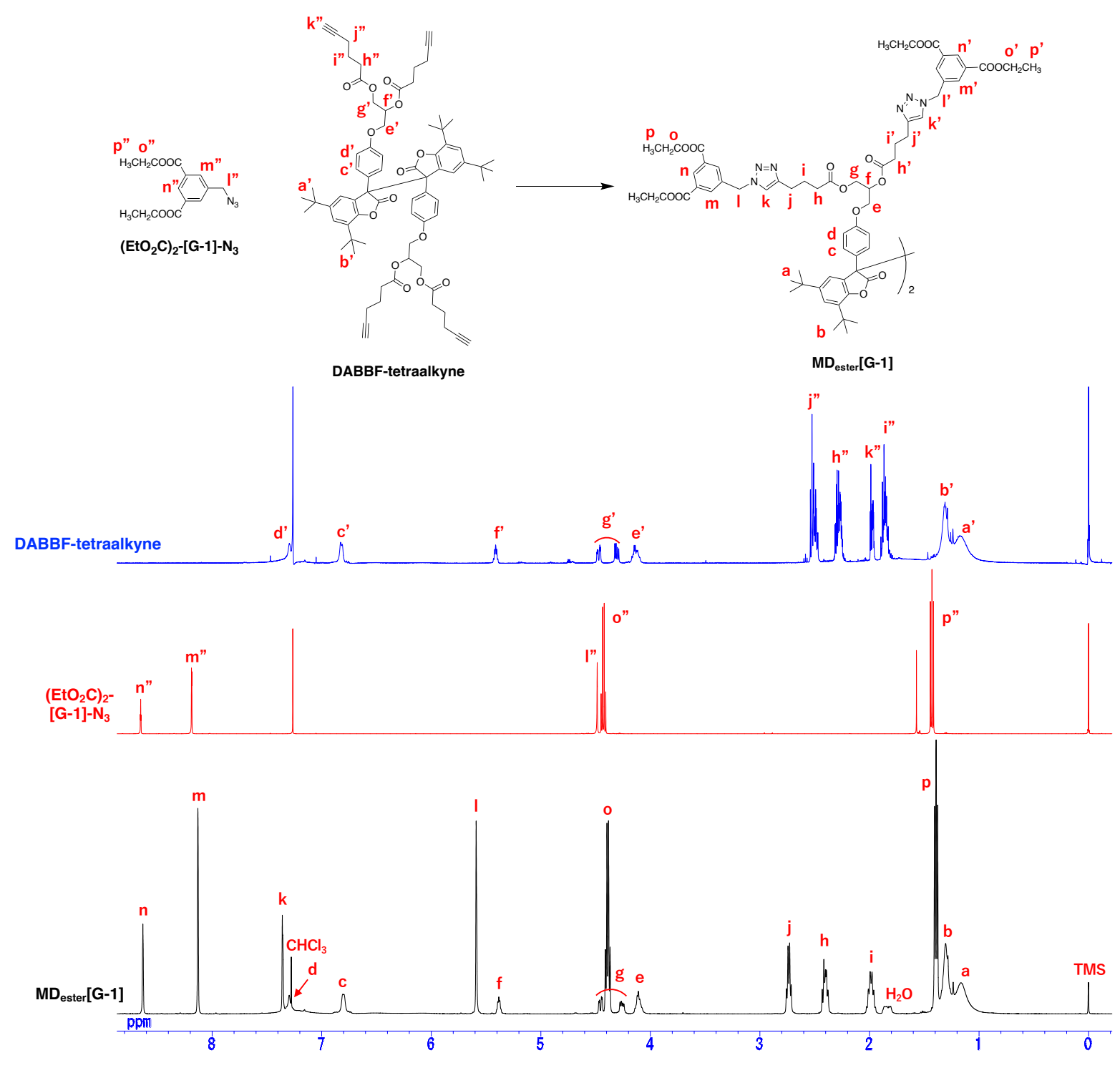

Figure S17. ${ }^{1} \mathrm{H}$ NMR spectra of $\mathbf{M D}_{\text {ester[ }}$ G-1] (black), $\left(\mathbf{E t O}_{2} \mathbf{C}\right)_{2}$-[G-1]-N $\mathbf{N}_{3}$ (red), and DABBF-tetraalkyne (blue) (500 MHz, $\left.\mathrm{CDCl}_{3}\right)$. 


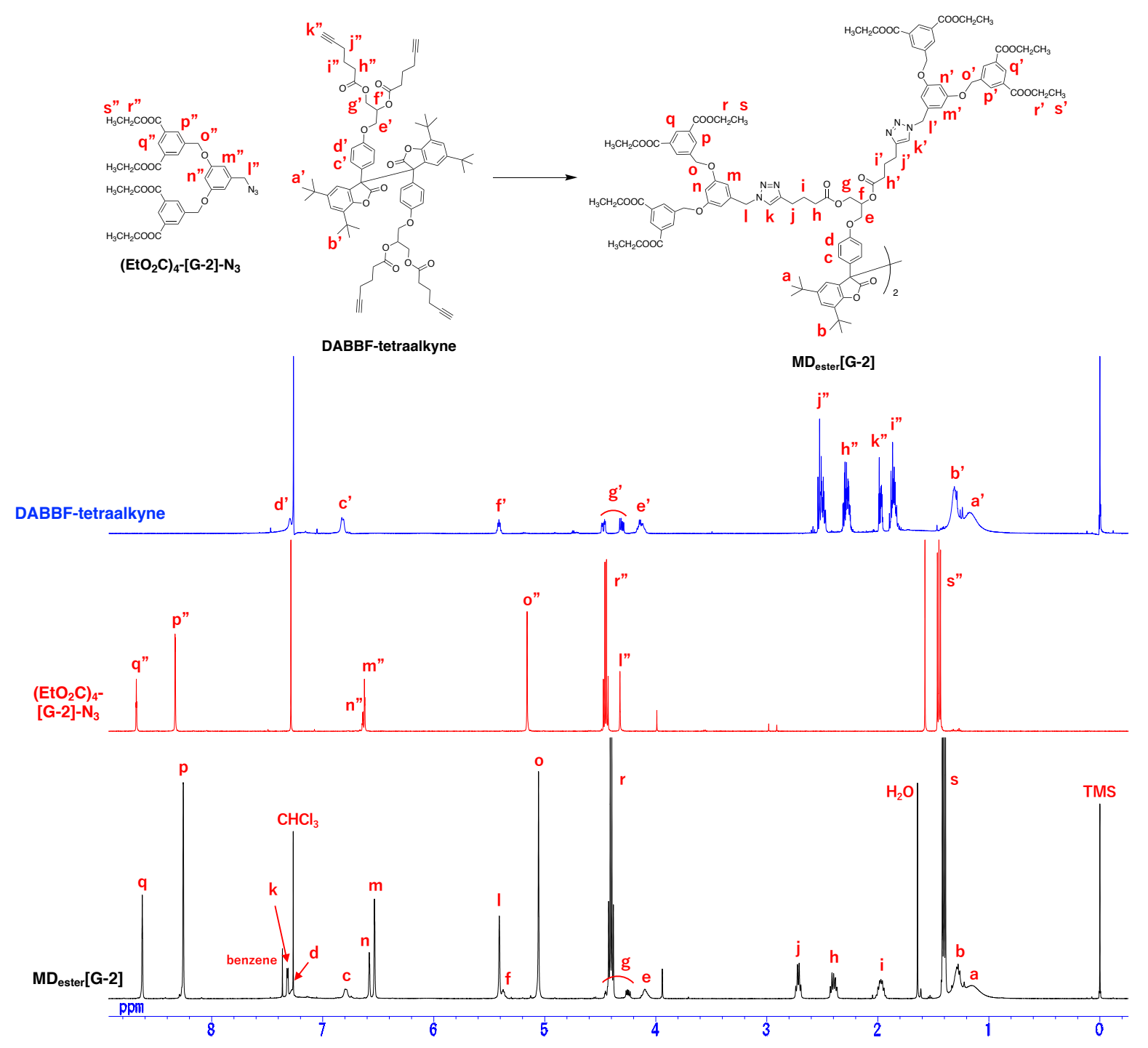

Figure S18. ${ }^{1} \mathrm{H}$ NMR spectra of $\mathbf{M D}_{\text {ester }}[\mathbf{G}-2]$ (black), $\left(\mathbf{E t O}_{2} \mathbf{C}\right)_{4}$-[G-2]-N $\mathbf{N}_{3}$ (red), and DABBF-tetraalkyne (blue) (500 MHz, $\left.\mathrm{CDCl}_{3}\right)$. 


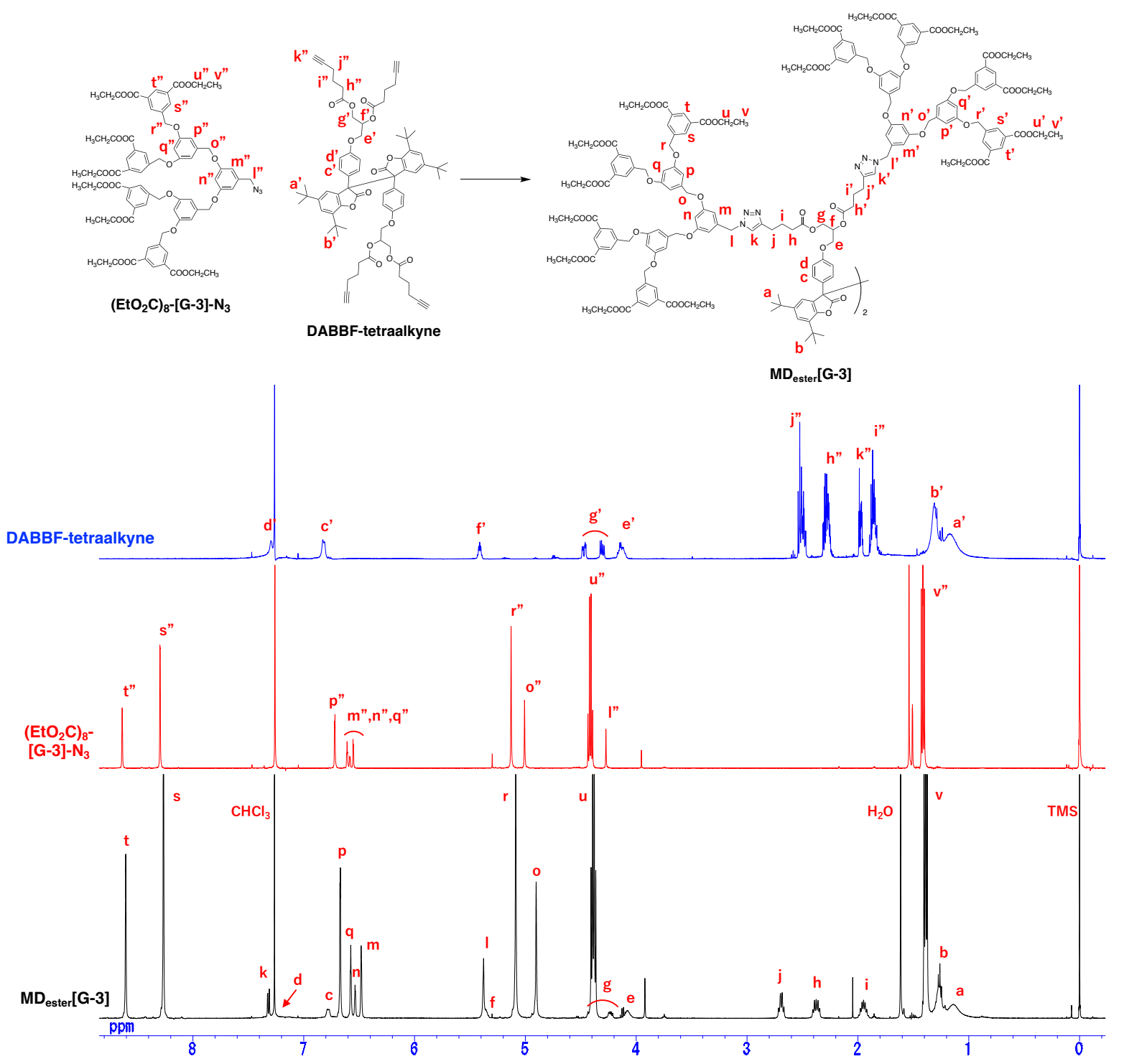

Figure S19. ${ }^{1} \mathrm{H}$ NMR spectra of $\mathbf{M D}_{\text {ester[ }}[\mathrm{G}-3]$ (black), $\left(\mathbf{E t O}_{2} \mathbf{C}\right)_{8}-[\mathbf{G}-3]-\mathbf{N}_{3}$ (red), and DABBF-tetraalkyne (blue) (500 MHz, $\left.\mathrm{CDCl}_{3}\right)$. 


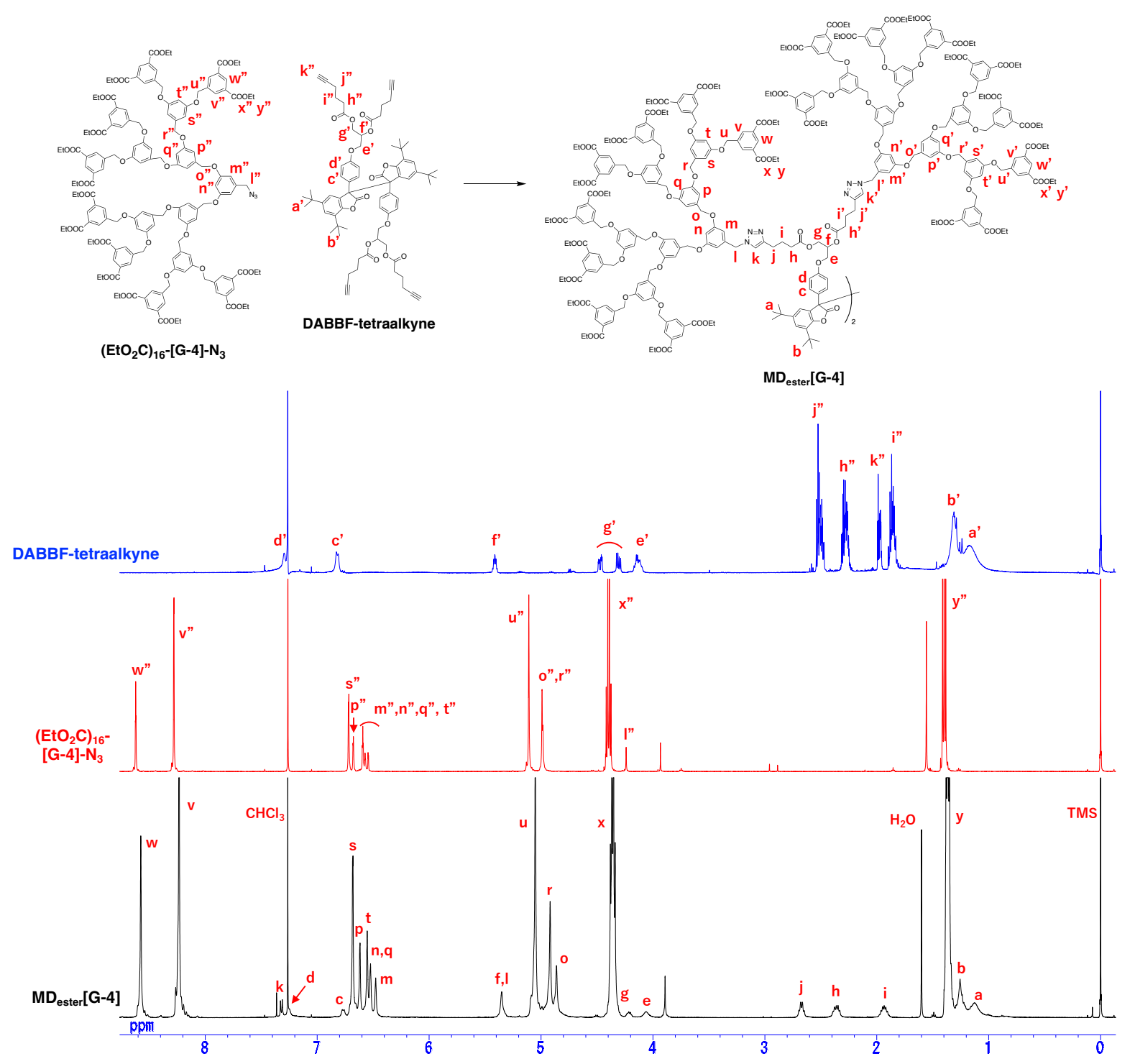

Figure S20. ${ }^{1} \mathrm{H}$ NMR spectra of $\mathbf{M D}_{\text {ester }}[\mathrm{G}-4]$ (black), $\left(\mathbf{E t O}_{2} \mathbf{C}\right)_{16-[G-4]-N_{3}}$ (red), and DABBF-tetraalkyne (blue) (500 MHz, $\left.\mathrm{CDCl}_{3}\right)$. 


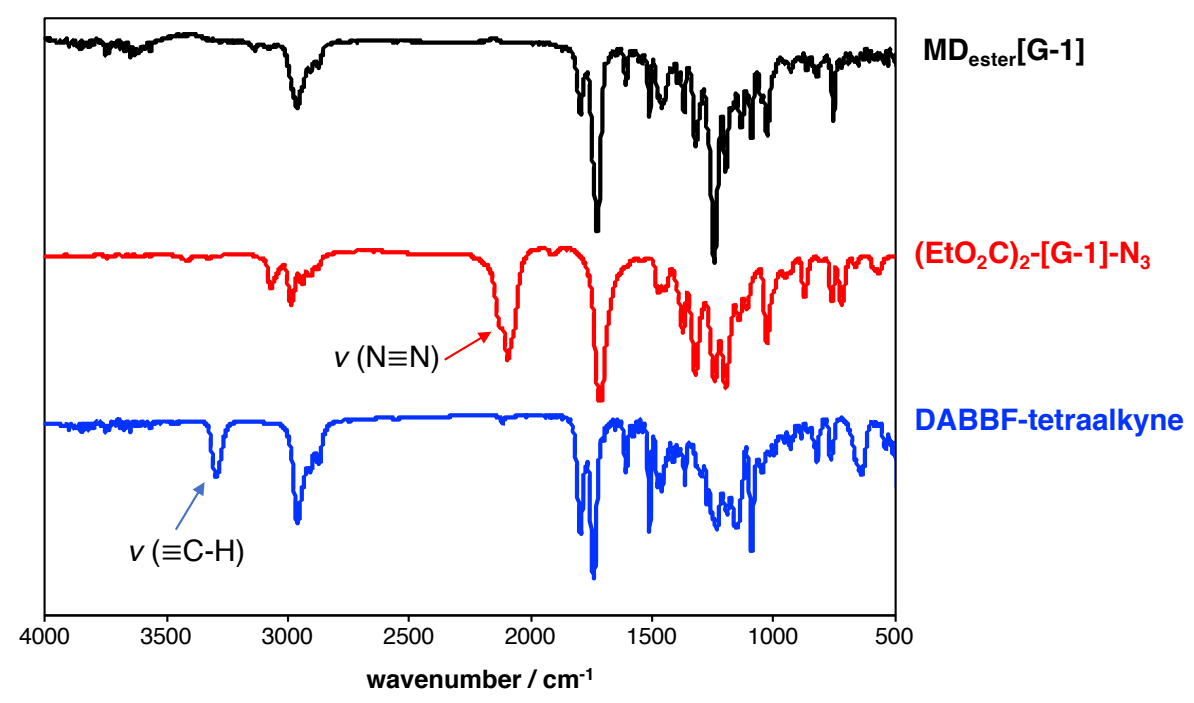

Figure S21. IR spectra of $\mathbf{M D}_{\text {ester}}[\mathrm{G}-1]$ (black), $\left(\mathbf{E t O}_{2} \mathbf{C}\right)_{2}-[\mathbf{G}-1]-\mathbf{N}_{3}$ (red), and DABBF-tetraalkyne (blue) $(\mathrm{KBr})$.

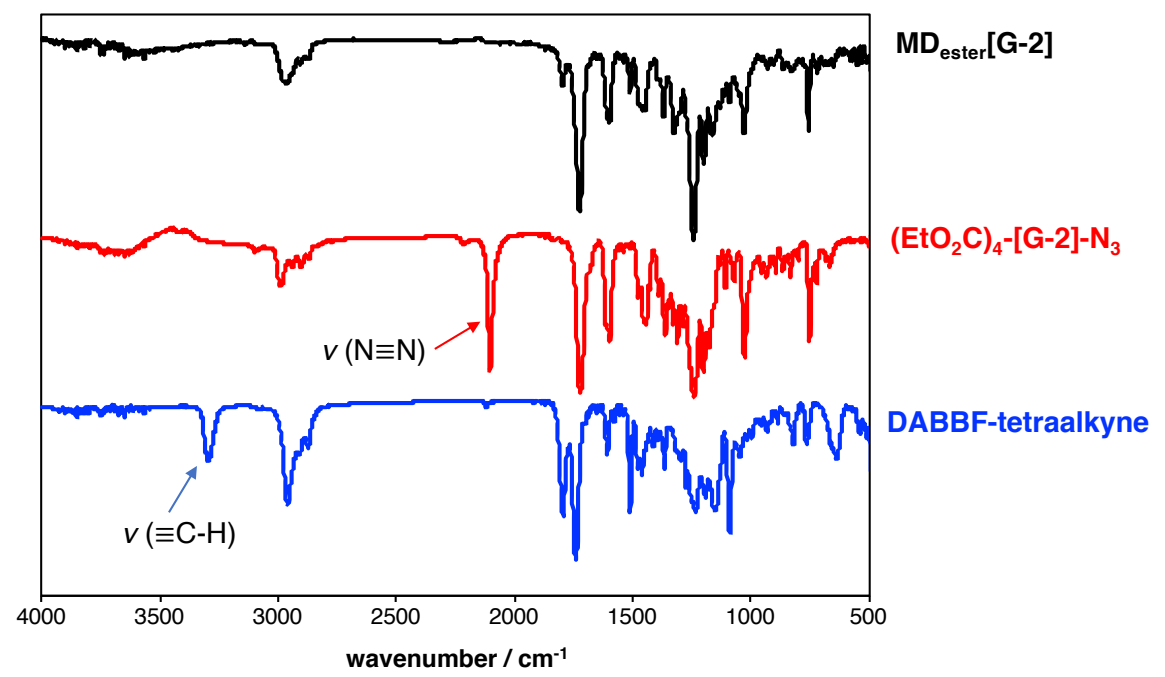

Figure S22. IR spectra of $\mathbf{M D}_{\text {ester }}[\mathbf{G}-2]$ (black), $\left(\mathbf{E t O}_{2} \mathbf{C}\right)_{4}-[\mathbf{G}-2]-\mathbf{N}_{3}$ (red), and DABBF-tetraalkyne (blue) $(\mathrm{KBr})$. 


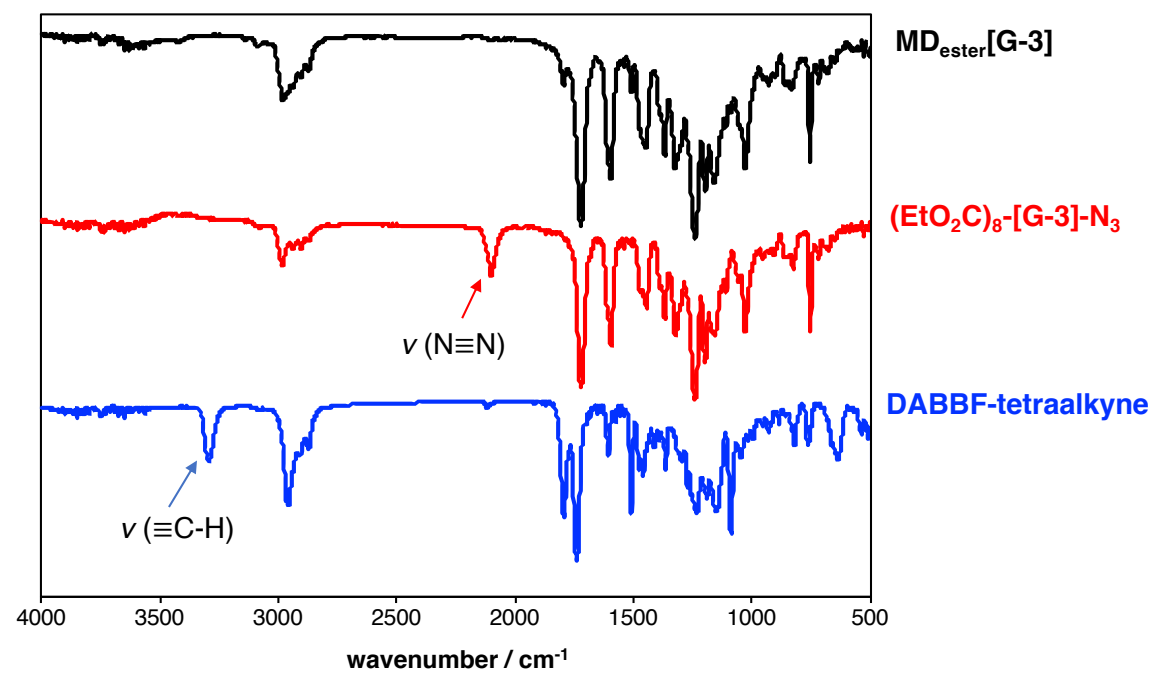

Figure S23. IR spectra of $\mathbf{M D}_{\text {ester }}[\mathrm{G}-3]$ (black), $\left(\mathbf{E t O}_{2} \mathbf{C}\right)_{8}-[\mathrm{G}-3]-\mathbf{N}_{3}$ (red), and DABBF-tetraalkyne (blue) $(\mathrm{KBr})$.

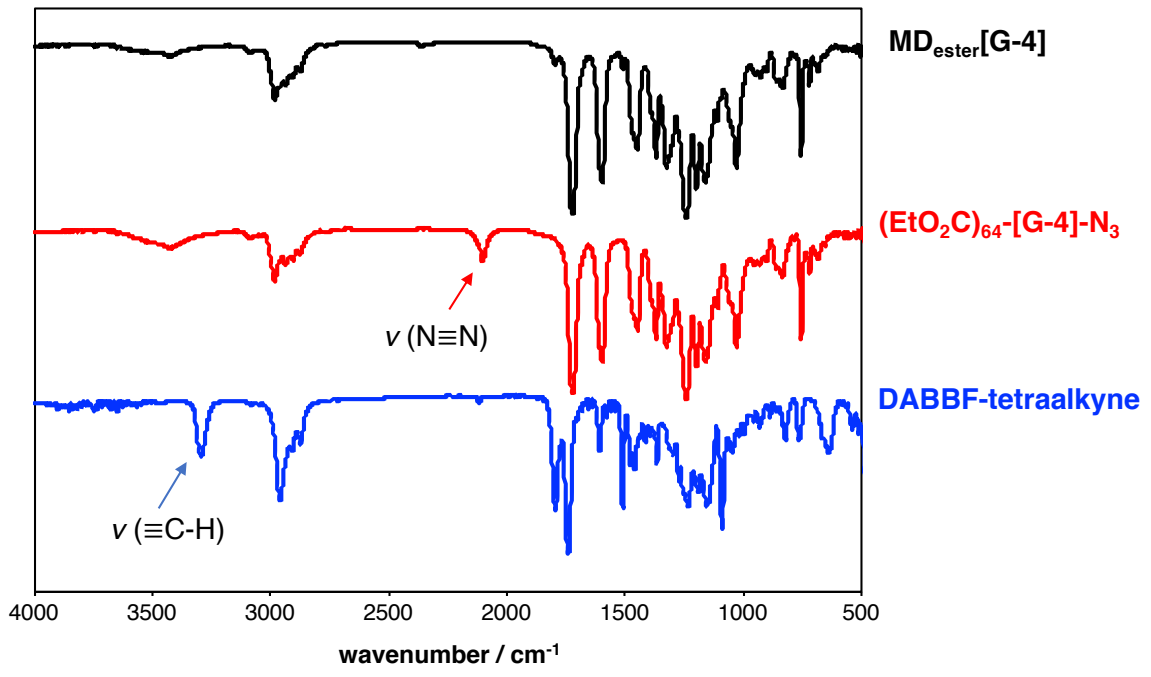

Figure S24. IR spectra of $\mathbf{M D}_{\text {ester}}[\mathrm{G}-4]$ (black), $\left(\mathrm{EtO}_{2} \mathbf{C}_{16-}-[\mathrm{G}-4]-\mathbf{N}_{3}\right.$ (red), and DABBF-tetraalkyne (blue) $(\mathrm{KBr})$. 
a)

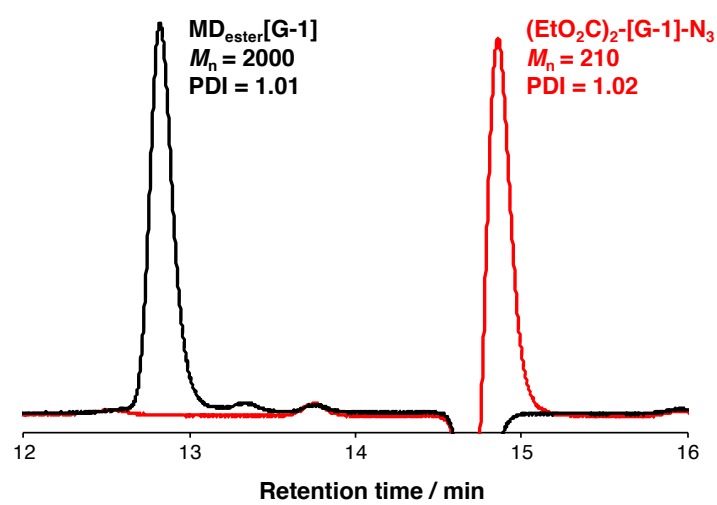

b)

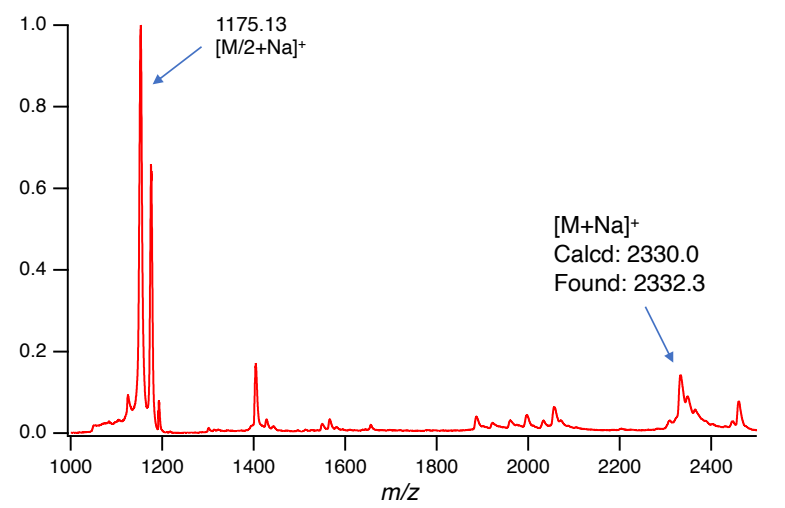

Figure S25. (a) Overlay of GPC traces for $\mathbf{M D}_{\text {ester }}[\mathbf{G}-\mathbf{1}]$ and $\left(\mathbf{E t O}_{\mathbf{2}} \mathbf{C}\right)_{2}-[\mathbf{G}-\mathbf{1}]-\mathbf{N}_{3}$. (b) MALDI-TOF-MS spectrum of $\mathbf{M D}_{\text {ester }}[\mathbf{G}-1]$ (DCTB / NaTFA).

a)

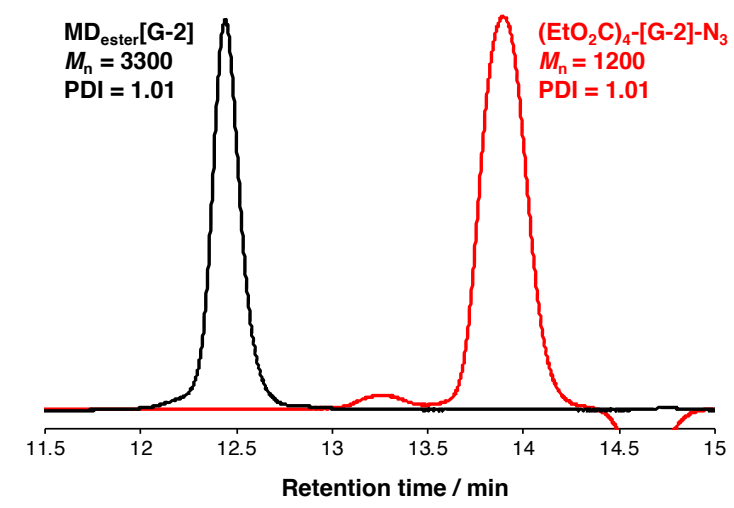

b)

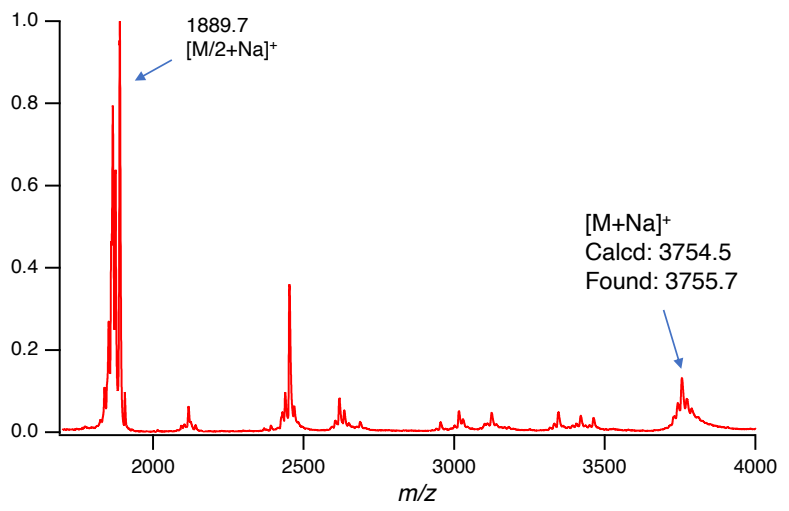

Figure S26. (a) Overlay of GPC traces for $\mathbf{M D}_{\text {ester }}[\mathbf{G}-2]$ and $\left(\mathbf{E t O}_{2} \mathbf{C}\right)_{4}-[\mathbf{G}-2]-\mathbf{N}_{3}$. (b) MALDI-TOF-MS spectrum of $\mathbf{M D}_{\text {ester}}[\mathbf{G}-2]$ (DCTB / NaTFA).

a)

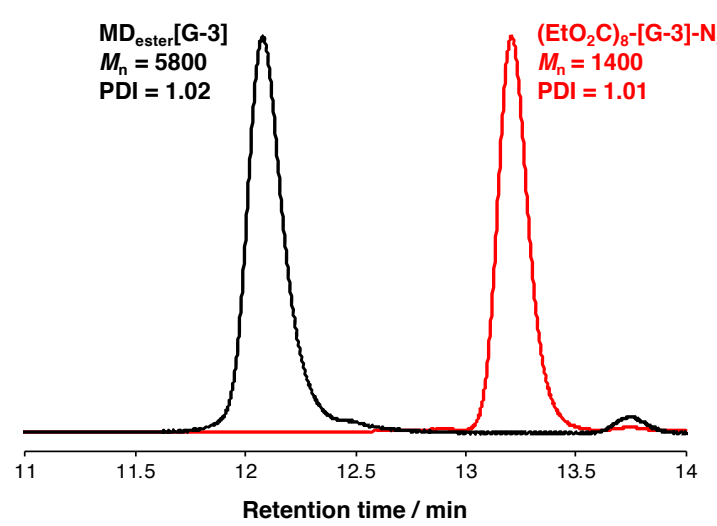

b)

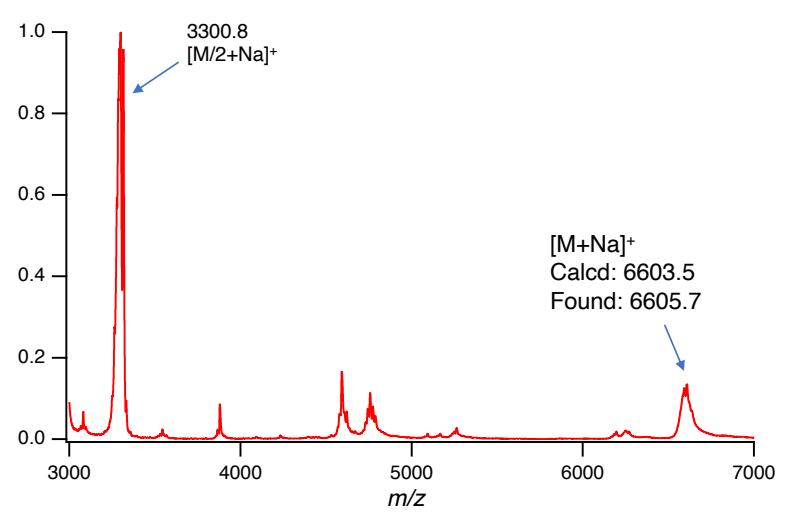

Figure S27. (a) Overlay of GPC traces for $\mathbf{M D}_{\text {ester }}[\mathbf{G}-3]$ and $\left(\mathbf{E t O}_{2} \mathbf{C}\right)_{8}-[\mathbf{G}-3]-\mathbf{N}_{3}$. (b) MALDI-TOF-MS spectrum of $\mathbf{M D}_{\text {ester }}[\mathbf{G}-3]$ (DCTB / NaTFA). 
a)

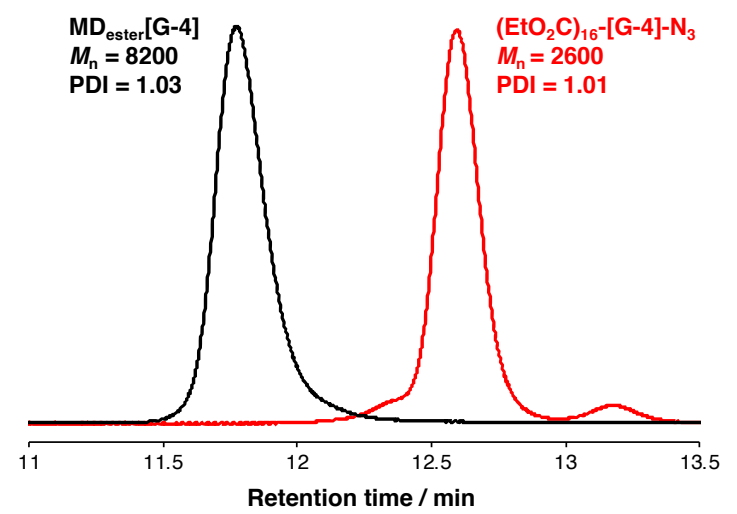

b)

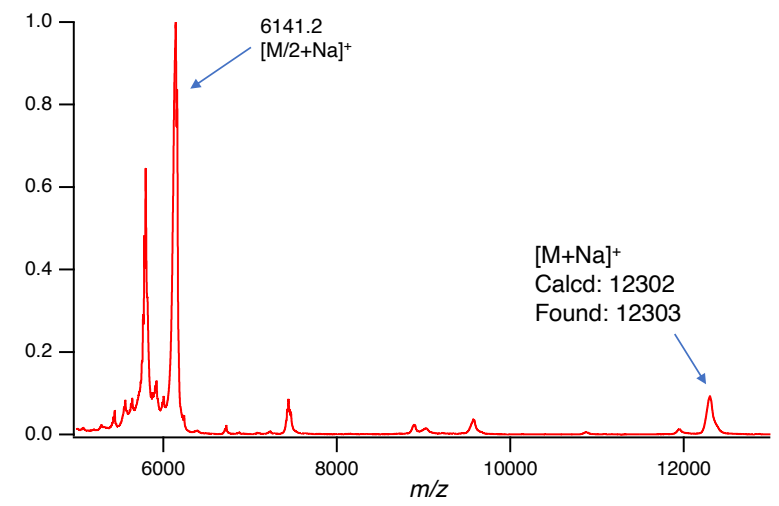

Figure S28. (a) Overlay of GPC traces for $\mathbf{M D}_{\text {ester }}[\mathbf{G}-4]$ and $\left(\mathbf{E t O}_{\mathbf{2}} \mathbf{C}\right)_{\mathbf{1 6}}-[\mathbf{G}-4]-\mathbf{N}_{\mathbf{3}}$. (b) MALDI-TOF-MS spectrum of $\mathbf{M D}_{\text {ester}}[\mathbf{G}-4]$ (DCTB / NaTFA). 


\subsection{Synthesis of $M D_{\text {amide }}$}

\section{MDamide[G-1]}
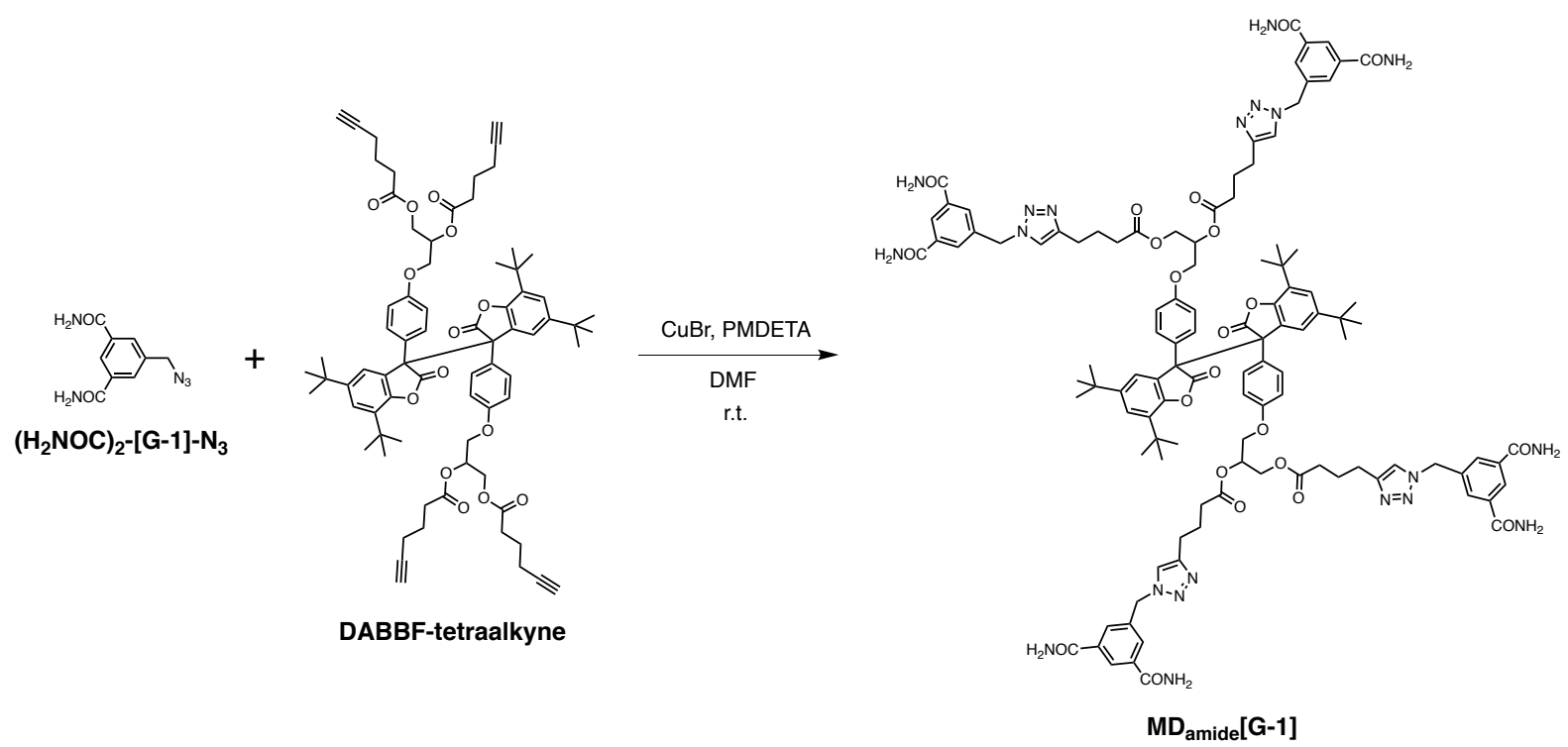

Under a nitrogen atmosphere, PMDETA $(0.106 \mathrm{~mL}, 0.508 \mathrm{mmol})$ was added to a solution of $\left(\mathbf{H}_{2} \mathbf{N O C}\right)_{2}$-[G1]-N $\mathbf{N}_{3}(0.558 \mathrm{~g}, 2.55 \mathrm{mmol})$, DABBF-tetraalkyne (0.600 g, $\left.0.500 \mathrm{mmol}\right)$, and $\mathrm{CuBr}(71.2 \mathrm{mg}, 0.496 \mathrm{mmol})$ in anhydrous DMF $(25 \mathrm{~mL})$ bubbled with nitrogen for 20 minutes. After being bubbled with nitrogen for another 20 minutes, the resulting solution was allowed to stir at r.t. for $3 \mathrm{~h}$. Following precipitation twice into a saturated aqueous solution of ethylenediamine-tetraacetic acid disodium salt $(\mathrm{EDTA} \cdot 2 \mathrm{Na})$ from DMF solution, the product was collected by centrifugation from aqueous suspension, and dried in vacuo to afford $\mathbf{M D}_{\text {amide }}[\mathbf{G}-1]$ as a white solid $(0.857 \mathrm{~g}, 83 \%)$.

${ }^{1} \mathrm{H}$ NMR (500 MHz, DMSO- $d_{6}$ ): $\delta /$ ppm 8.29 (s, 4H, aromatic), 8.03 (s, 8H, NH), 7.94 (s, 4H, aromatic), $7.93\left(\mathrm{~s}, 8 \mathrm{H}\right.$, aromatic), $7.51(\mathrm{~s}, 8 \mathrm{H}, \mathrm{NH}), 7.35-5.96\left(\mathrm{~m}, 8 \mathrm{H}\right.$, aromatic), $5.60\left(\mathrm{~s}, 8 \mathrm{H}, \mathrm{CH}_{2}\right), 5.33(\mathrm{br}, 2 \mathrm{H}, \mathrm{CH})$, 4.36-4.18 (m, 8H, $\mathrm{CH}_{2}$ ), 2.60 (m, 8H, $\left.\mathrm{CH}_{2}\right), 2.35$ (m, 8H, $\mathrm{CH}_{2}$ ), 1.81 (m, 8H, $\mathrm{CH}_{2}$ ), 1.32-1.03 (br, 36H, $\left.\mathrm{CH}_{3}\right)^{13} \mathrm{C}$ NMR (100 MHz, DMSO- $\left.d_{6}\right): \delta /$ ppm 172.48, 172.20, 167.29, 162.41, 158.32, 148.27, 146.63, 136.57, 135.03, 132.94, 129.96, 126.22, 122.32, 113.75, 69.30, 66.36, 62.12, 52.42, 35.88, 34.47, 34.05, 33.00, 32.78, 31.13, 29.49, 29.44, 29.40, 29.34, 24.32, 24.27, 24.22 ; FT-IR (KBr, $\left.\mathrm{cm}^{-1}\right): 3357,3195,2958$, $1794,1738,1671,1595,1509,1460,1366,1231,1134,1090,1053,820,761,628$; ESI-TOF: $\mathrm{m} / z[\mathrm{M}+2 \mathrm{Na}]^{2+}$ calcd: 1060.94 , found: 1060.94 . 

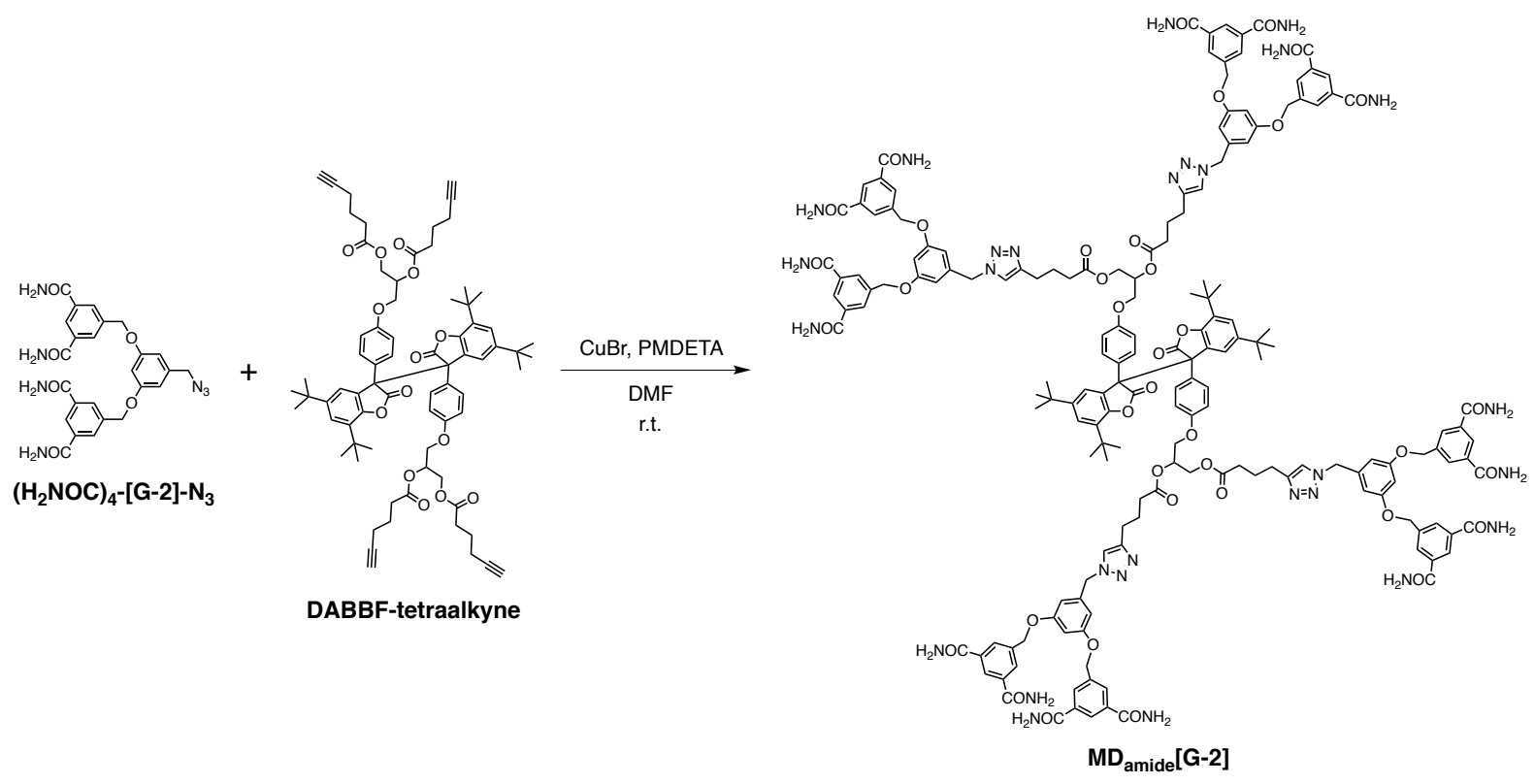

Under a nitrogen atmosphere, PMDETA $(0.108 \mathrm{~mL}, 0.515 \mathrm{mmol})$ was added to a solution of $\left(\mathbf{H}_{2} \mathbf{N O C}\right)_{4}$-[G2]-N $\mathbf{3}$ (0.240 g, $0.463 \mathrm{mmol})$, DABBF-tetraalkyne ( $0.124 \mathrm{~g}, 0.103 \mathrm{mmol})$, and $\mathrm{CuBr}$ (43.8 mg, $0.305 \mathrm{mmol}$ ) in anhydrous DMF $(8 \mathrm{~mL})$ bubbled with nitrogen for 20 minutes. After being bubbled with nitrogen for another 20 minutes, the resulting solution was allowed to stir at r.t. for $4 \mathrm{~h}$. The product was precipitated three times into a saturated aqueous solution of EDTA $\cdot 2 \mathrm{Na}$ and once into methanol from DMF, and dried in vacuo at $40{ }^{\circ} \mathrm{C}$ to afford $\mathbf{M D}_{\text {amide }}[\mathbf{G}-2]$ as a white solid $(0.237 \mathrm{~g}, 70 \%)$.

${ }^{1} \mathrm{H}$ NMR (500 MHz, DMSO- $d_{6}$ ): $\delta / \mathrm{ppm} 8.32$ (s, 8H, aromatic), 8.07 (s, 16H, aromatic), 8.05 (s, 16H, NH), $7.88(\mathrm{~s}, 4 \mathrm{H}$, aromatic), $7.48(\mathrm{~s}, 16 \mathrm{H}, \mathrm{NH}), 7.31-5.97$ (m, 8H, aromatic), $6.72(\mathrm{~s}, 4 \mathrm{H}$, aromatic), 6.61 (s, 8H, aromatic), $5.44\left(\mathrm{~s}, 8 \mathrm{H}, \mathrm{CH}_{2}\right), 5.34(\mathrm{~s}, 2 \mathrm{H}, \mathrm{CH}), 5.14\left(\mathrm{~s}, 16 \mathrm{H}, \mathrm{CH}_{2}\right), 4.36-4.19\left(\mathrm{~m}, 8 \mathrm{H}, \mathrm{CH}_{2}\right), 2.59(\mathrm{~m}, 8 \mathrm{H}$, $\mathrm{CH}_{2}$ ), $2.36\left(\mathrm{~m}, 8 \mathrm{H}, \mathrm{CH}_{2}\right), 1.81$ (m, 8H, $\left.\mathrm{CH}_{2}\right), 1.30-1.03$ (br, 36H, $\left.\mathrm{CH}_{3}\right) ;{ }^{13} \mathrm{C}$ NMR (100 MHz, DMSO- $\left.d_{6}\right): \delta$ / ppm 172.49, 172.22, 167.45, 159.62, 158.33, 148.26, 146.54, 138.50, 137.24, 134.74, 132.94, 129.48, $126.27,122.20,113.77,107.32,101.31,69.28,69.00,62.14,52.74,34.42,34.04,33.02,32.79,31.12,29.43$, 24.25; FT-IR (KBr, cm $\left.{ }^{-1}\right): 3361,2957,1790,1729,1666,1594,1510,1445,1369,1232,1166,1091,765$, 627; ESI-TOF: $m / z[\mathrm{M}+2 \mathrm{Na}]^{2+}$ calcd: 1657.64 , found: 1657.64 . 


\section{MDamide[G-3]}
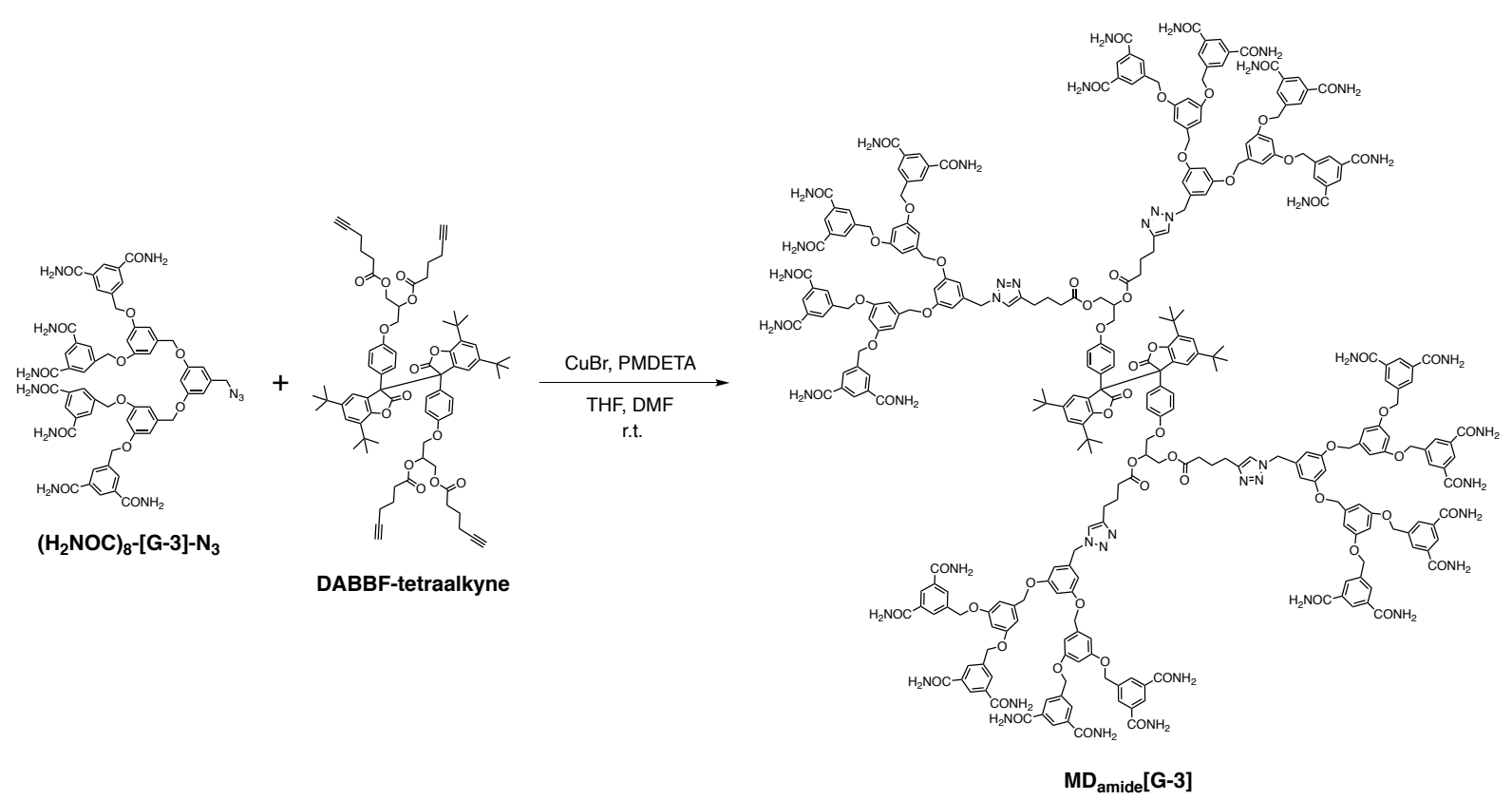

Under a nitrogen atmosphere, PMDETA $(0.131 \mathrm{~mL}, 0.627 \mathrm{mmol})$ was added to a solution of $\left(\mathbf{H}_{2} \mathbf{N O C}\right)_{8}$-[G3]-N 3 (0.698 g, $0.627 \mathrm{mmol})$, DABBF-tetraalkyne $(0.151 \mathrm{~g}, 0.126 \mathrm{mmol})$, and $\mathrm{CuBr}$ (74.4 mg, $0.519 \mathrm{mmol})$ in anhydrous DMF $(20 \mathrm{~mL})$ bubbled with nitrogen for 20 minutes. After being bubbled with nitrogen for another 20 minutes, the resulting solution was allowed to stir at r.t. for $2 \mathrm{~h}$. The product was precipitated twice into a saturated aqueous solution of EDTA $\cdot 2 \mathrm{Na}$, once each into ethanol/acetone $=1: 2$ and ethanol/THF $=2: 1$ from DMF, and dried in vacuo at $40{ }^{\circ} \mathrm{C}$ to afford MD $_{\text {amide }}[\mathbf{G}-3]$ as a white solid $(0.554 \mathrm{~g}, 78 \%)$.

${ }^{1} \mathrm{H}$ NMR (500 MHz, DMSO- $d_{6}$ ): $\delta /$ ppm 8.32 (s, 16H, aromatic), 8.08 (s, 32H, aromatic), 8.06 (s, 32H, NH), $7.90(\mathrm{~s}, 4 \mathrm{H}$, aromatic), $7.48(\mathrm{~s}, 32 \mathrm{H}, \mathrm{NH}), 7.29-5.94(\mathrm{~m}, 8 \mathrm{H}$, aromatic), $6.74(\mathrm{~s}, 16 \mathrm{H}$, aromatic), $6.71(\mathrm{~s}, 8 \mathrm{H}$, aromatic), $6.64\left(\mathrm{~s}, 4 \mathrm{H}\right.$, aromatic), $6.56\left(\mathrm{~s}, 8 \mathrm{H}\right.$, aromatic), $5.43\left(\mathrm{~s} 8 \mathrm{H}, \mathrm{CH}_{2}\right), 5.31(\mathrm{~s}, 2 \mathrm{H}, \mathrm{CH}), 5.17(\mathrm{~s}, 32 \mathrm{H}$, $\left.\mathrm{CH}_{2}\right), 4.98$ (s, 16H, $\left.\mathrm{CH}_{2}\right), 4.34-4.17\left(\mathrm{~m}, 8 \mathrm{H}, \mathrm{CH}_{2}\right), 2.58\left(\mathrm{~m}, 8 \mathrm{H}, \mathrm{CH}_{2}\right), 2.34\left(\mathrm{~m}, 8 \mathrm{H}, \mathrm{CH}_{2}\right), 1.80(\mathrm{~m}, 8 \mathrm{H}$, $\mathrm{CH}_{2}$ ), 1.29-1.00 (br, 36H, $\left.\mathrm{CH}_{3}\right) ;{ }^{13} \mathrm{C}$ NMR (100 MHz, DMSO- $\left.d_{6}\right): \delta / \mathrm{ppm} 172.55,172.27,167.59,159.73$, 159.52, 148.30, 146.60, 139.43, 138.41, 137.50, 134.77, 129.53, 126.29, 122.33, 107.20, 106.81101 .34 , $101.19,69.33,69.00,52.82,34.45,34.06,33.06,32.81,31.40,31.15,29.47,24.29$; FT-IR $\left(\mathrm{KBr}_{\mathrm{cm}}{ }^{-1}\right): 3354$, 2957, 1666, 1594, 1510, 1446, 1376, 1161, 1057, 895, 833, 764, 631; ESI-TOF: $m / z[\mathrm{M}+2 \mathrm{Na}]^{2+}$ calcd: 2850.52, found: $2850.52 ;[\mathrm{M}+3 \mathrm{Na}]^{3+}$ calcd: 1908.01 , found: 1908.01 . 


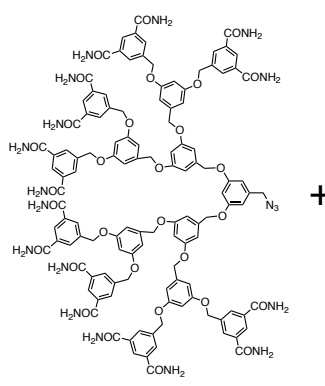

$\left(\mathrm{H}_{2} \mathrm{NOC}\right)_{16}-[\mathrm{G}-4]-\mathrm{N}_{3}$
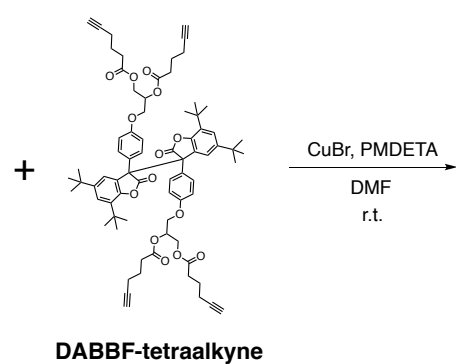

DABBF-tetraalkyn

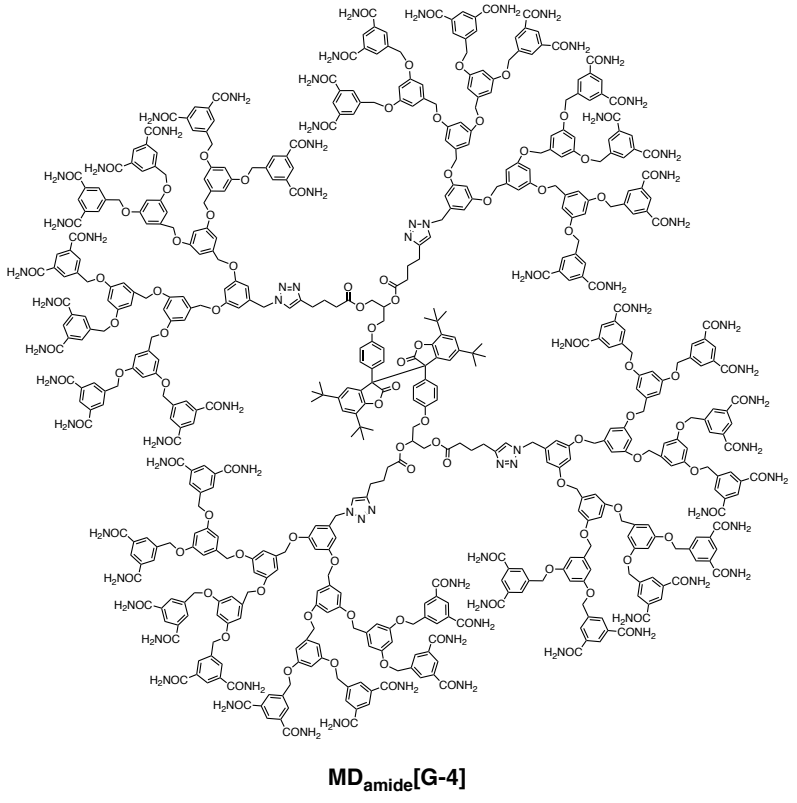

$M D_{\text {amide }}[\mathrm{G}-4]$

Under a nitrogen atmosphere, PMDETA $(0.106 \mathrm{~mL}, 0.506 \mathrm{mmol})$ was added to a solution of $\left(\mathbf{H}_{2} \mathbf{N O C}\right)_{16^{-}}$ [G-4]-N $\mathbf{3}$ (0.700 g, $0.303 \mathrm{mmol})$, DABBF-tetraalkyne (60.9 mg, $50.8 \mu \mathrm{mol})$, and $\mathrm{CuBr}(72.8 \mathrm{mg}, 0.507$ mmol) in anhydrous DMF ( $25 \mathrm{~mL})$ bubbled with nitrogen for 20 minutes. After being bubbled with nitrogen for another 20 minutes, the resulting solution was allowed to stir at r.t. for $3 \mathrm{~h}$. The product was precipitated twice into a saturated aqueous solution of EDTA $\cdot 2 \mathrm{Na}$, once each into ethanol and methanol/acetone $=4: 1$ from DMF, and dried in vacuo at $40{ }^{\circ} \mathrm{C}$ to afford $\mathbf{M D}_{\text {amide }}[\mathbf{G}-4]$ as a white solid $(0.415 \mathrm{~g}, 78 \%)$.

${ }^{1} \mathrm{H}$ NMR (500 MHz, DMSO- $d_{6}$ ): $\delta /$ ppm 8.32 (s, 32H, aromatic), 8.08 (s, 128H, aromatic and NH), 7.90 (s, $4 \mathrm{H}$, aromatic), $7.51(\mathrm{~s}, 64 \mathrm{H}, \mathrm{NH}), 7.26-5.91(\mathrm{~m}, 8 \mathrm{H}$, aromatic), $6.76(\mathrm{~s}, 32 \mathrm{H}$, aromatic), $6.70(\mathrm{~s}, 32 \mathrm{H}$, aromatic), 6.63 (s, 12H, aromatic), $6.57\left(\mathrm{~s}, 8 \mathrm{H}\right.$, aromatic), $5.42\left(\mathrm{br}, 8 \mathrm{H}, \mathrm{CH}_{2}\right), 5.30(\mathrm{~s}, 2 \mathrm{H}, \mathrm{CH}), 5.16(\mathrm{~s}, 64 \mathrm{H}$, $\mathrm{CH}_{2}$ ), 5.01 (br, 48H, $\left.\mathrm{CH}_{2}\right), 4.34-4.20\left(\mathrm{~m}, 8 \mathrm{H}, \mathrm{CH}_{2}\right), 2.57\left(\mathrm{~m}, 8 \mathrm{H}, \mathrm{CH}_{2}\right), 2.32\left(\mathrm{~m}, 8 \mathrm{H}, \mathrm{CH}_{2}\right), 1.78(\mathrm{~m}, 8 \mathrm{H}$, $\mathrm{CH}_{2}$ ), 1.26-0.96 (br, 36H, $\left.\mathrm{CH}_{3}\right) ;{ }^{13} \mathrm{C}$ NMR (100 MHz, DMSO- $\left.d_{6}\right): \delta / \mathrm{ppm} 167.51,159.76,159.58,159.48$, $146.55,139.54,139.18,137.43,134.72,129.48,126.25,106.78,101.24,69.27,68.96,31.09,29.35,24.25$; FT-IR (KBr, cm $\left.{ }^{-1}\right): 3353,3196,2950,1666,1594,1446,1371,1318,1158,1052,897,834,763,623$; MALDI-TOF: $m / z$ [M+Cu] $]^{+}$calcd: 10485 , found: 10484 . 

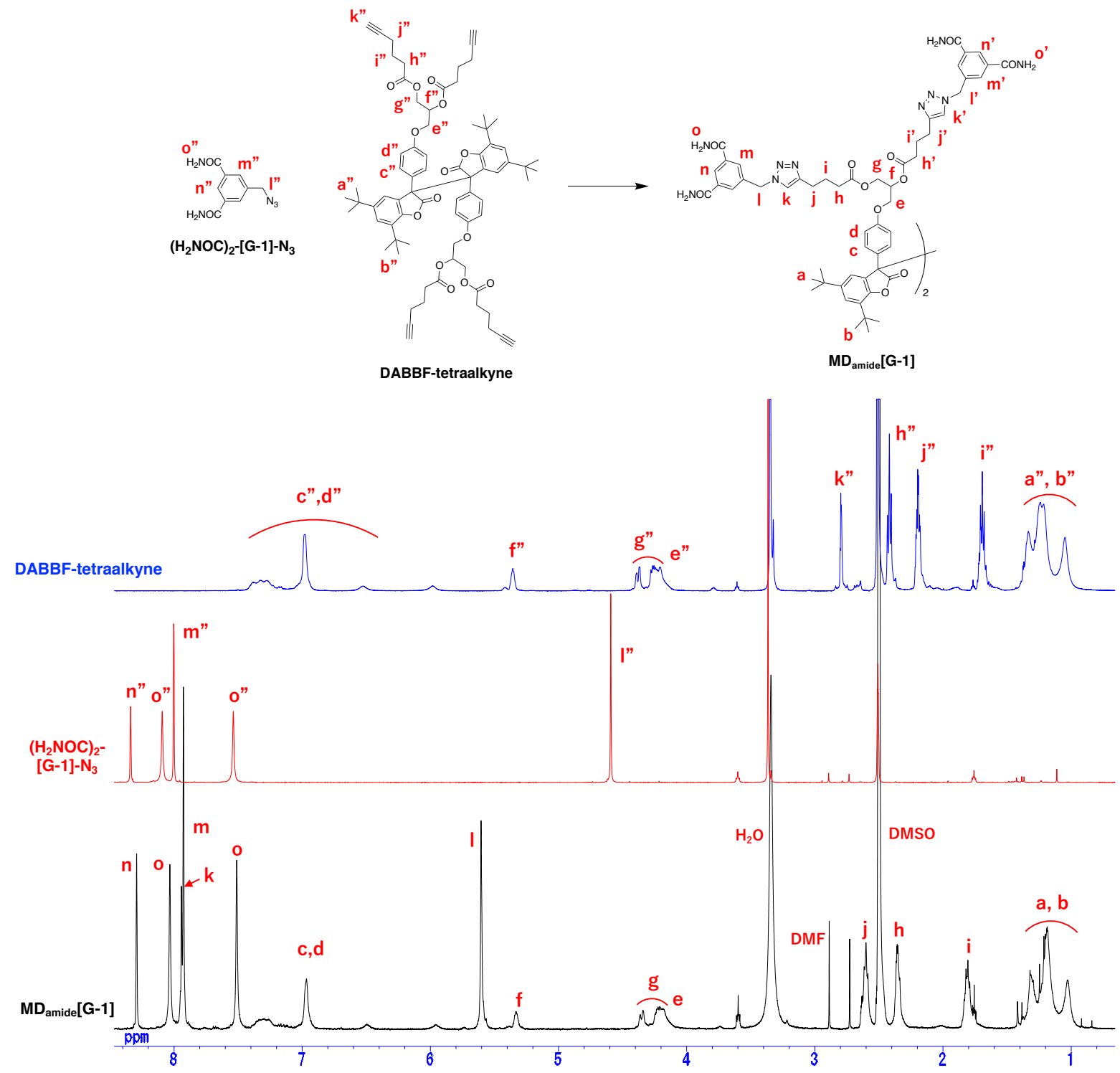

Figure S29. ${ }^{1} \mathrm{H}$ NMR spectra of $\mathbf{M D}_{\text {amide }}[\mathbf{G}-1]$ (black), ( $\left.\mathbf{H}_{2} \mathbf{N O C}\right)_{2}-[\mathbf{G}-1]-\mathbf{N}_{3}$ (red), and DABBFtetraalkyne (blue) (500 MHz, DMSO- $\left.d_{6}\right)$. 


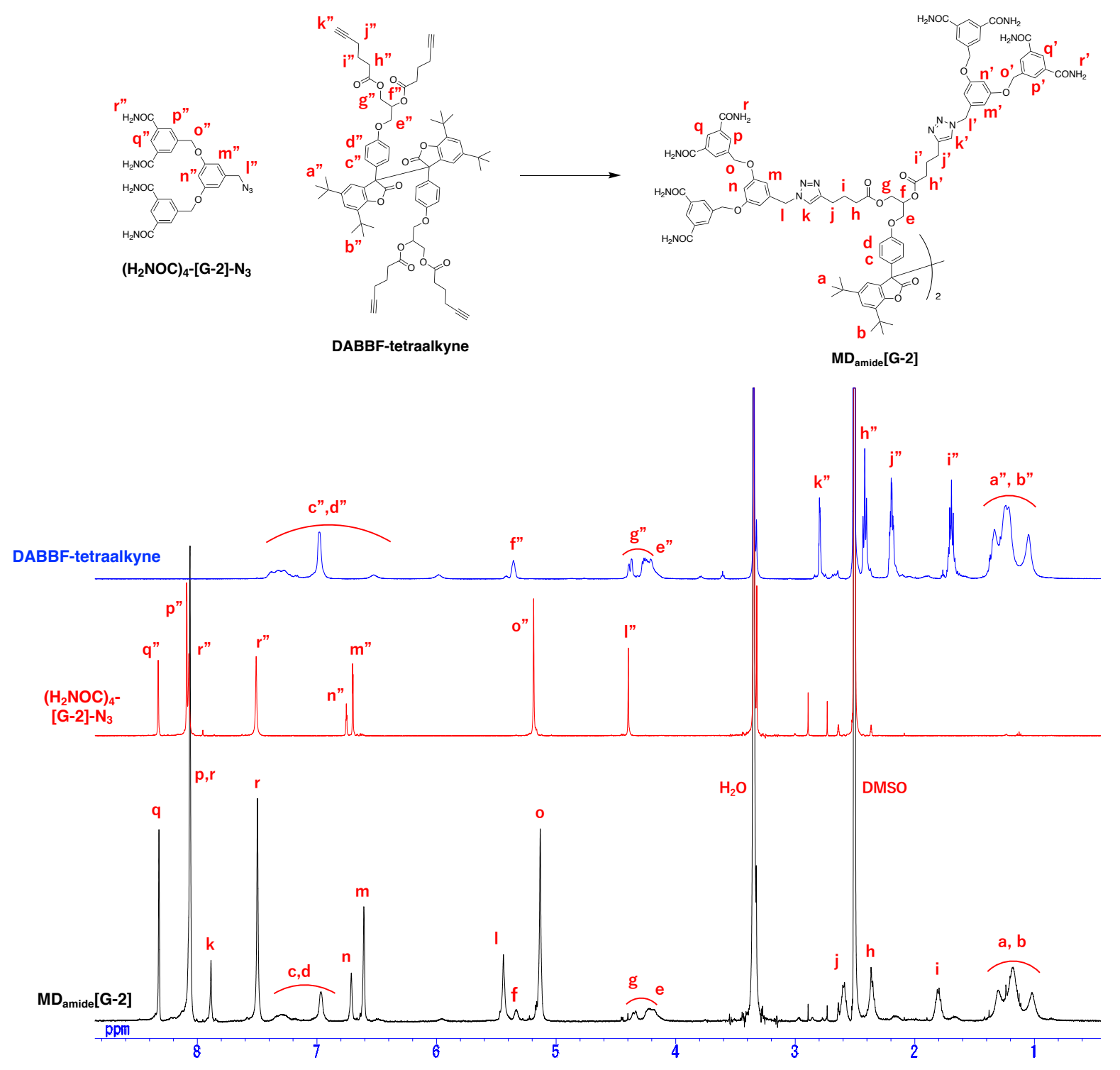

Figure S30. ${ }^{1} \mathrm{H}$ NMR spectra of $\mathbf{M D}_{\text {amide }}[\mathbf{G}-2]$ (black), ( $\left.\mathbf{H}_{2} \mathbf{N O C}\right)_{4}-[\mathbf{G}-2]-\mathbf{N}_{3}$ (red), and DABBFtetraalkyne (blue) (500 MHz, DMSO- $\left.d_{6}\right)$. 


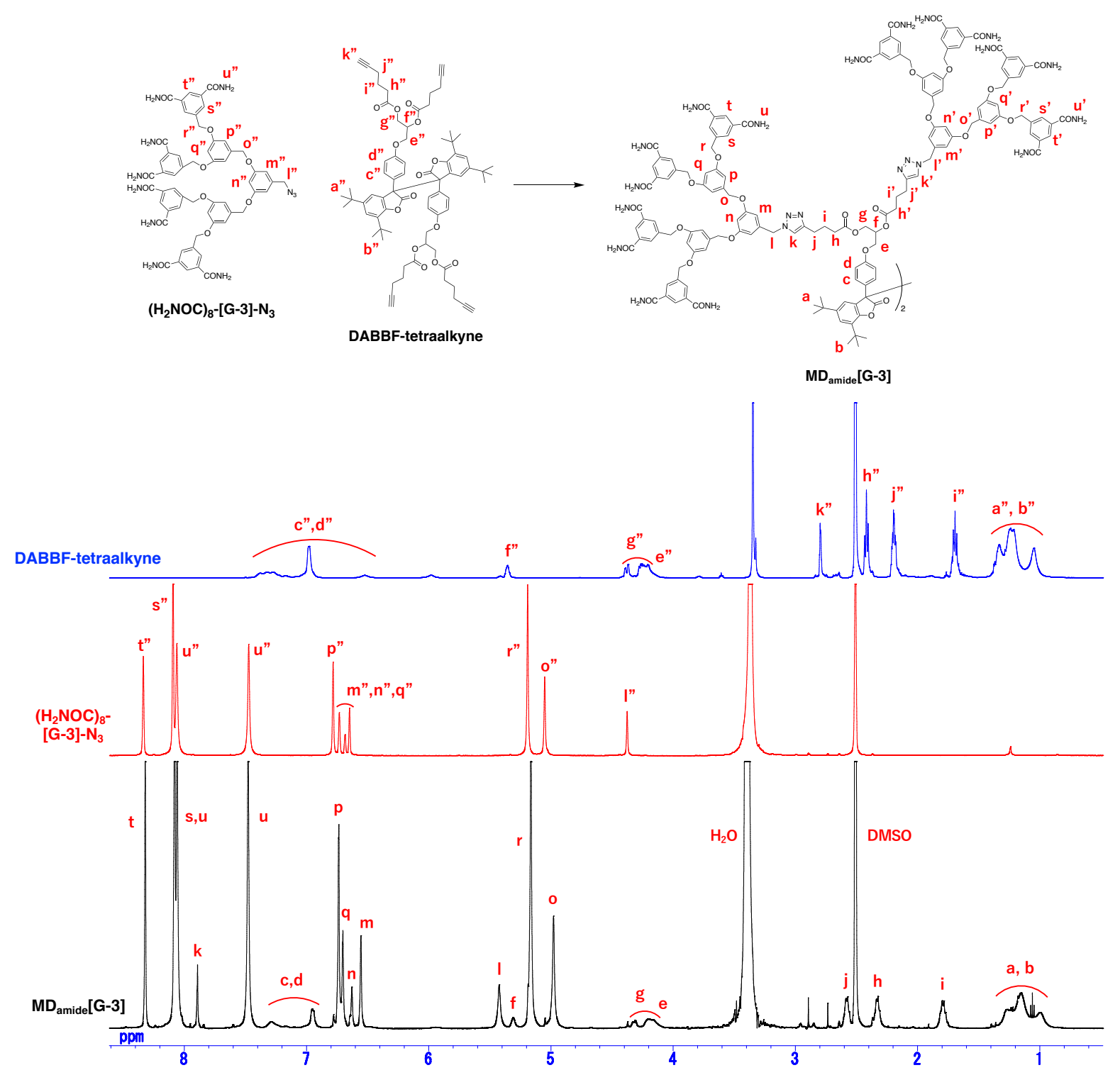

Figure S31. ${ }^{1} \mathrm{H}$ NMR spectra of $\mathbf{M D}_{\text {amide }}[\mathbf{G}-3]$ (black), $\left(\mathbf{H}_{2} \mathbf{N O C}\right)_{8}-[\mathbf{G}-3]-\mathbf{N}_{3}$ (red), and DABBFtetraalkyne (blue) (500 MHz, DMSO- $\left.d_{6}\right)$. 


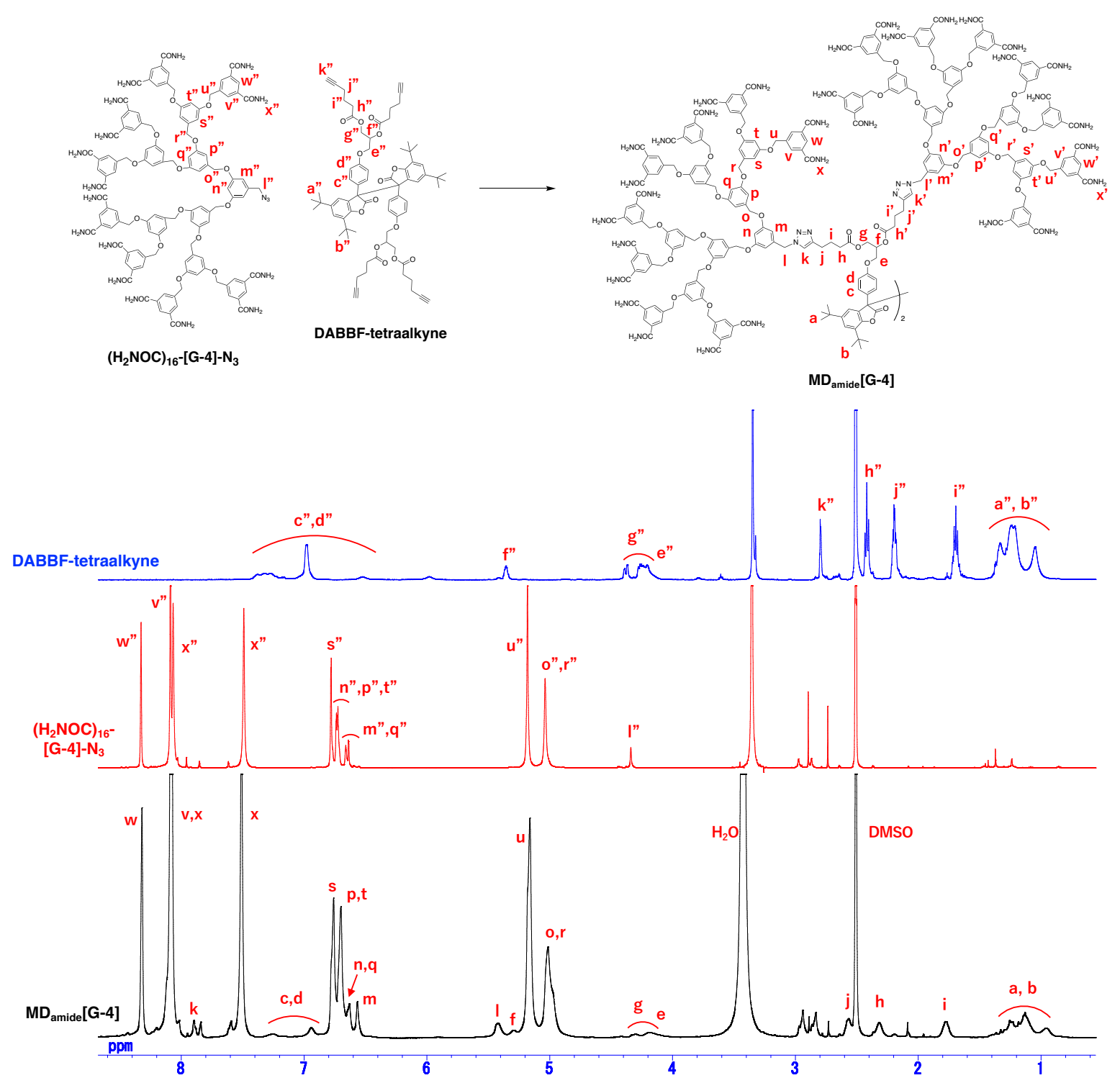

Figure S32. ${ }^{1} \mathrm{H}$ NMR spectra of $\mathbf{M D}_{\text {amide }}[\mathbf{G}-4]$ (black), $\left(\mathbf{H}_{2} \mathbf{N O C}\right)_{16-[G-4]-N_{3}}$ (red), and DABBFtetraalkyne (blue) $\left(500 \mathrm{MHz}, \mathrm{CDCl}_{3}\right)$. 


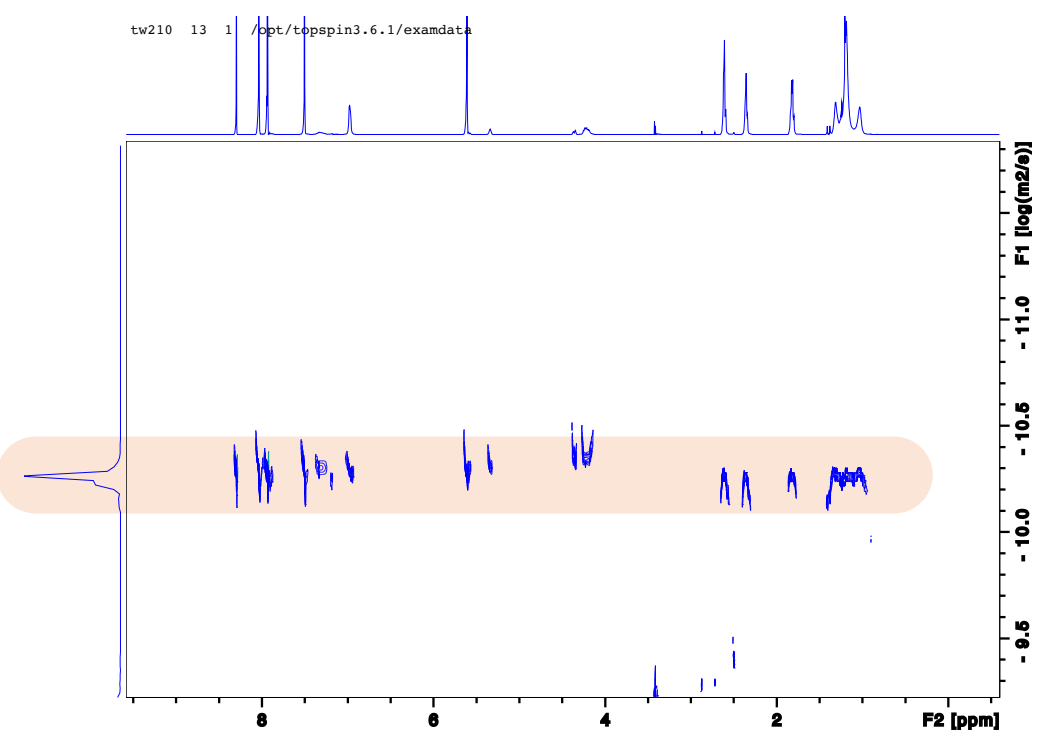

Figure S33. ${ }^{1} \mathrm{H}$ DOSY spectrum of $\mathbf{M D}_{\text {amide }}[\mathbf{G}-1]$ (500 MHz, DMSO- $\left.d_{6}\right)$.

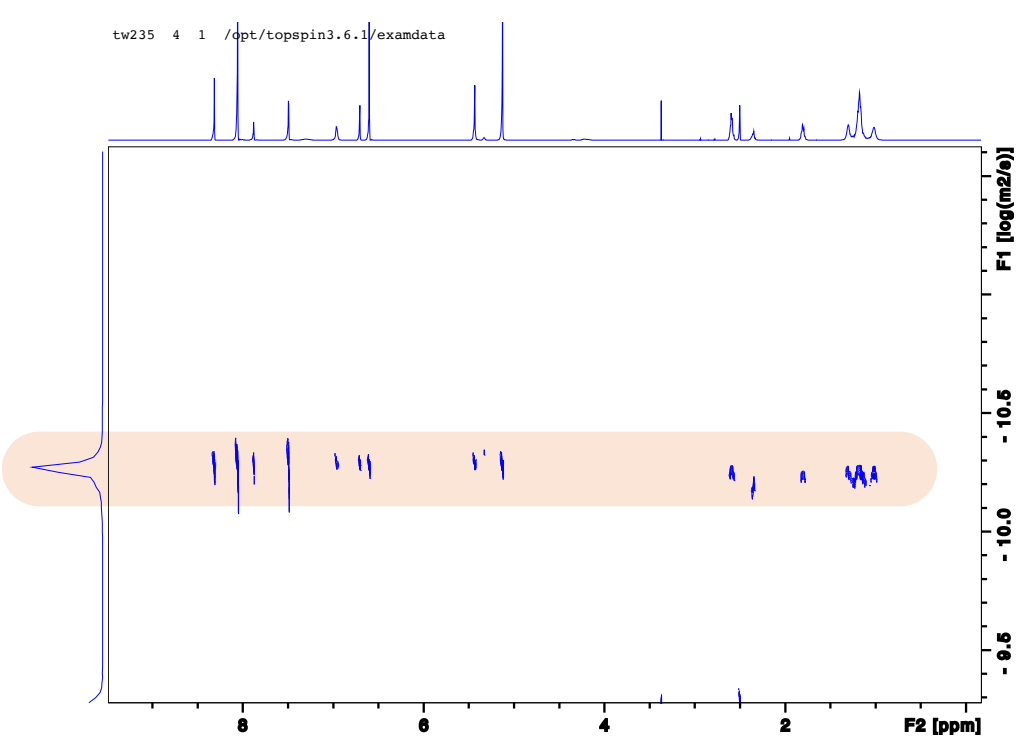

Figure S34. ${ }^{1} \mathrm{H}$ DOSY spectrum of $\mathbf{M D}_{\text {amide }}[\mathbf{G}-2]\left(500 \mathrm{MHz}, \mathrm{DMSO}-d_{6}\right)$. 


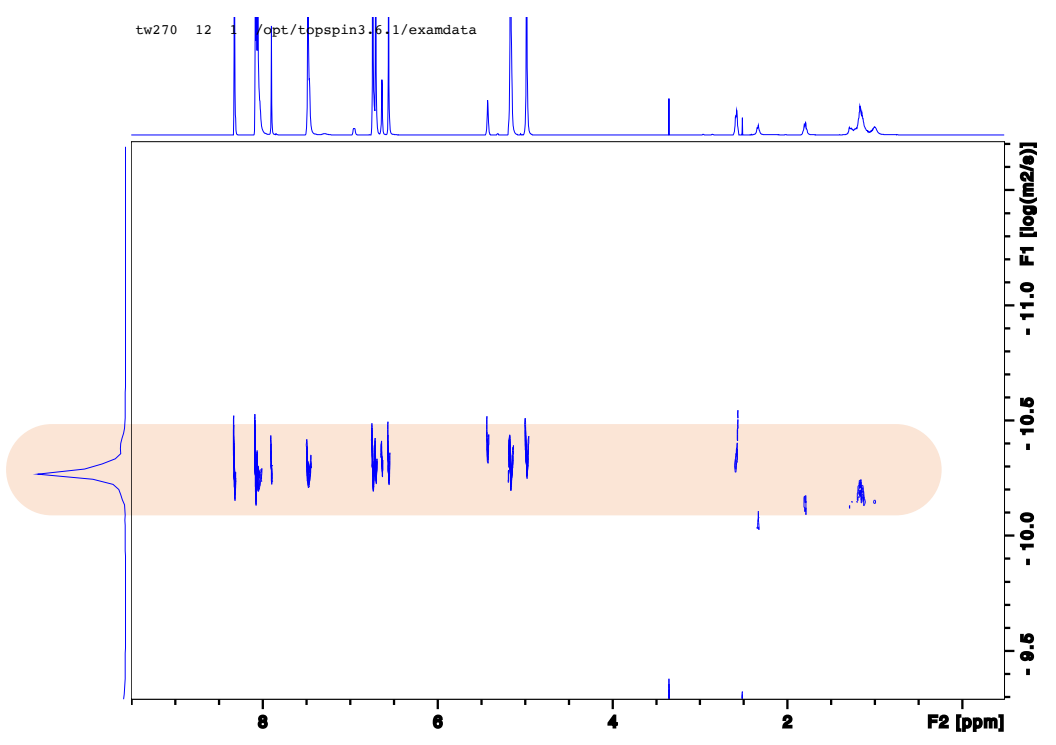

Figure S35. ${ }^{1} \mathrm{H}$ DOSY spectrum of $\mathbf{M D}_{\text {amide }}[\mathbf{G - 3}]\left(500 \mathrm{MHz}, \mathrm{DMSO}-d_{6}\right)$.

a)

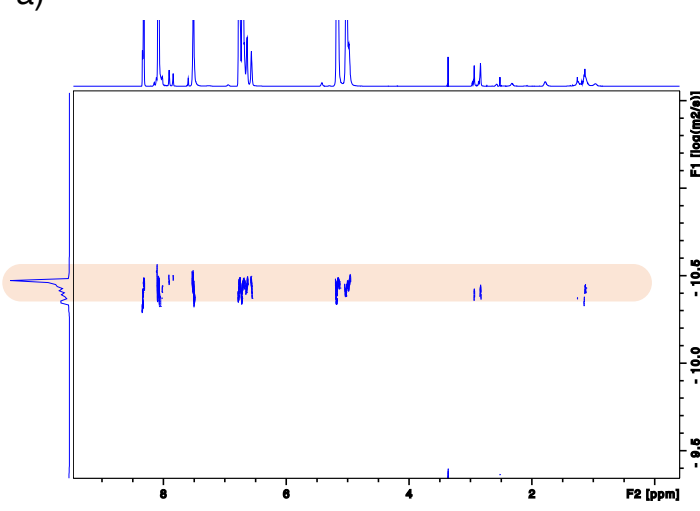

b)

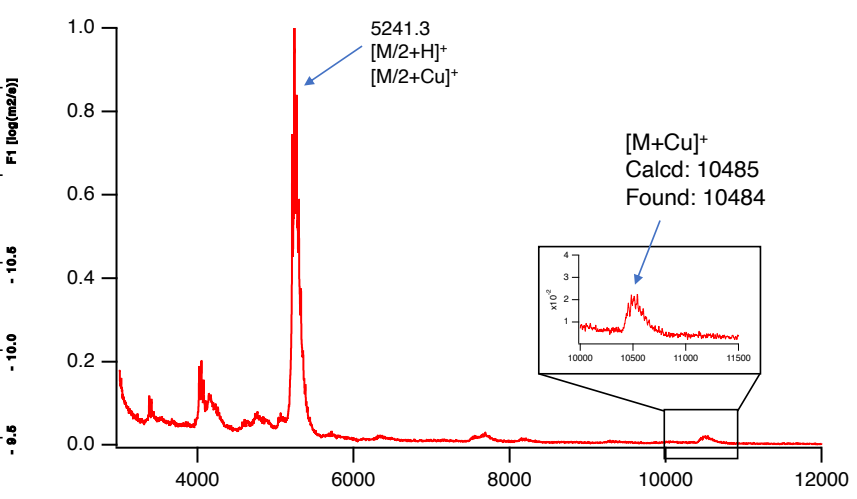

Figure S36. (a) ${ }^{1} \mathrm{H}$ DOSY spectrum of $\mathbf{M D}_{\text {amide }}$ [G-4]. (b) MALDI-TOF-MS spectrum of $\mathbf{M D}_{\text {amide }}[\mathbf{G}-4$ ] (Dithranol). 


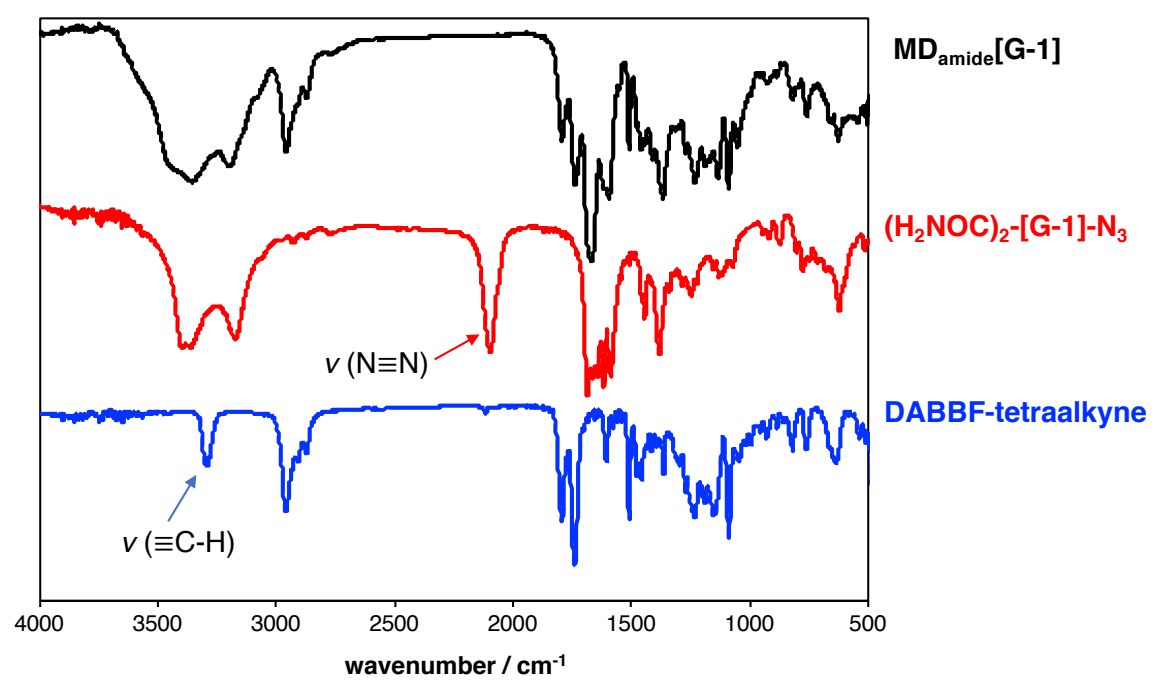

Figure S37. IR spectra of $\mathbf{M D}_{\text {amide }}[\mathrm{G}-1]$ (black), $\left(\mathrm{H}_{2} \mathbf{N O C}\right)_{2}$-[G-1]-N $\mathbf{N}_{3}$ (red), and DABBF-tetraalkyne (blue) $(\mathrm{KBr})$.

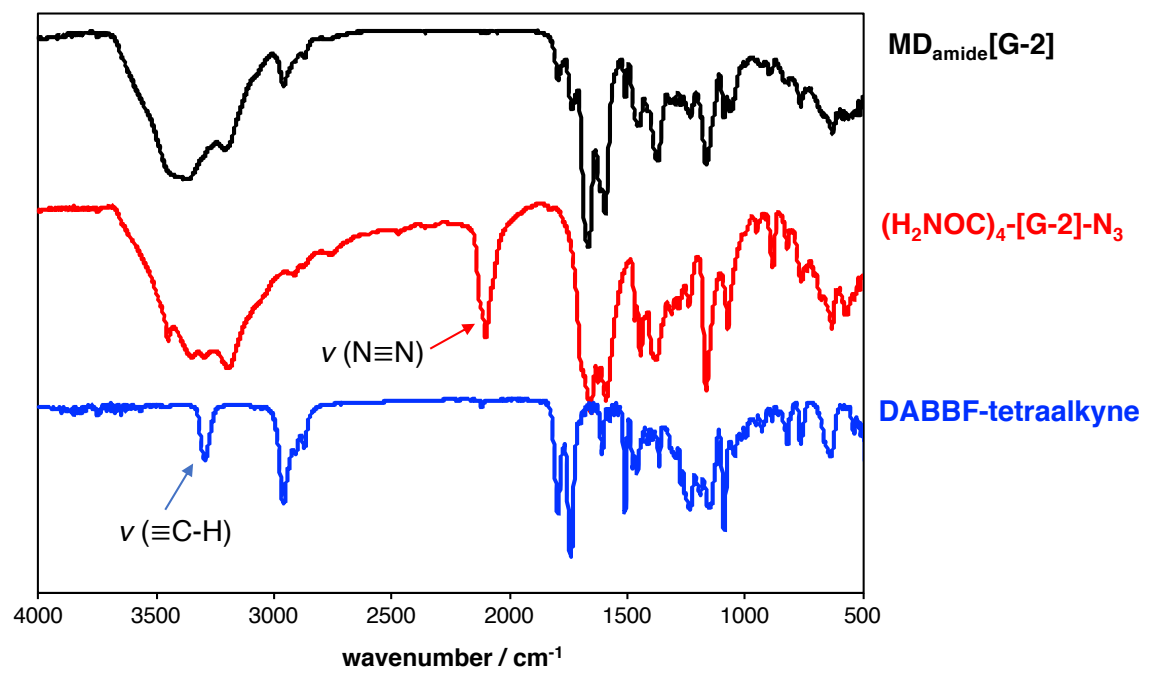

Figure S38. IR spectra of $\mathbf{M D}_{\text {amide }}[\mathrm{G}-2]$ (black), $\left(\mathrm{H}_{2} \mathbf{N O C}\right)_{4}-[\mathrm{G}-2]-\mathbf{N}_{3}$ (red), and DABBF-tetraalkyne (blue) $(\mathrm{KBr})$. 


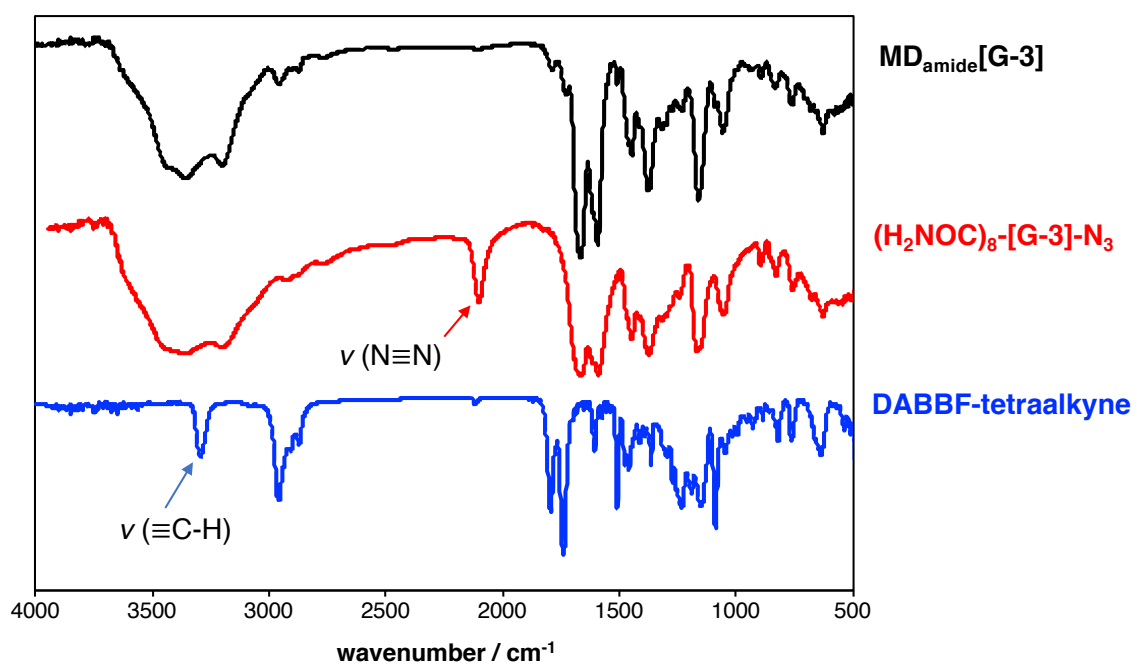

Figure S39. IR spectra of $\mathbf{M D}_{\text {amide }}[\mathrm{G}-3]$ (black), $\left(\mathrm{H}_{2} \mathbf{N O C}\right)_{8}$-[G-3]- $\mathbf{N}_{3}$ (red), and DABBF-tetraalkyne (blue) $(\mathrm{KBr})$.

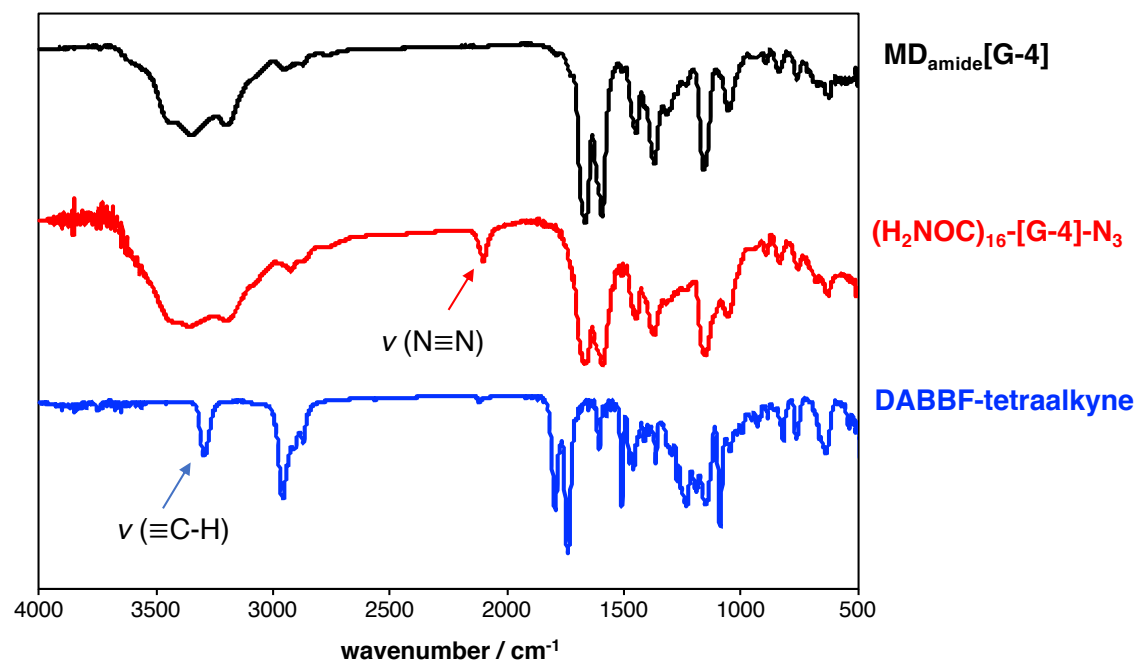

Figure S40. IR spectra of $\mathrm{MD}_{\text {amide }}[\mathrm{G}-4]$ (black), $\left(\mathrm{H}_{2} \mathbf{N O C}\right)_{16-[G-4]-N_{3}}$ (red), and DABBF-tetraalkyne (blue) $(\mathrm{KBr})$. 

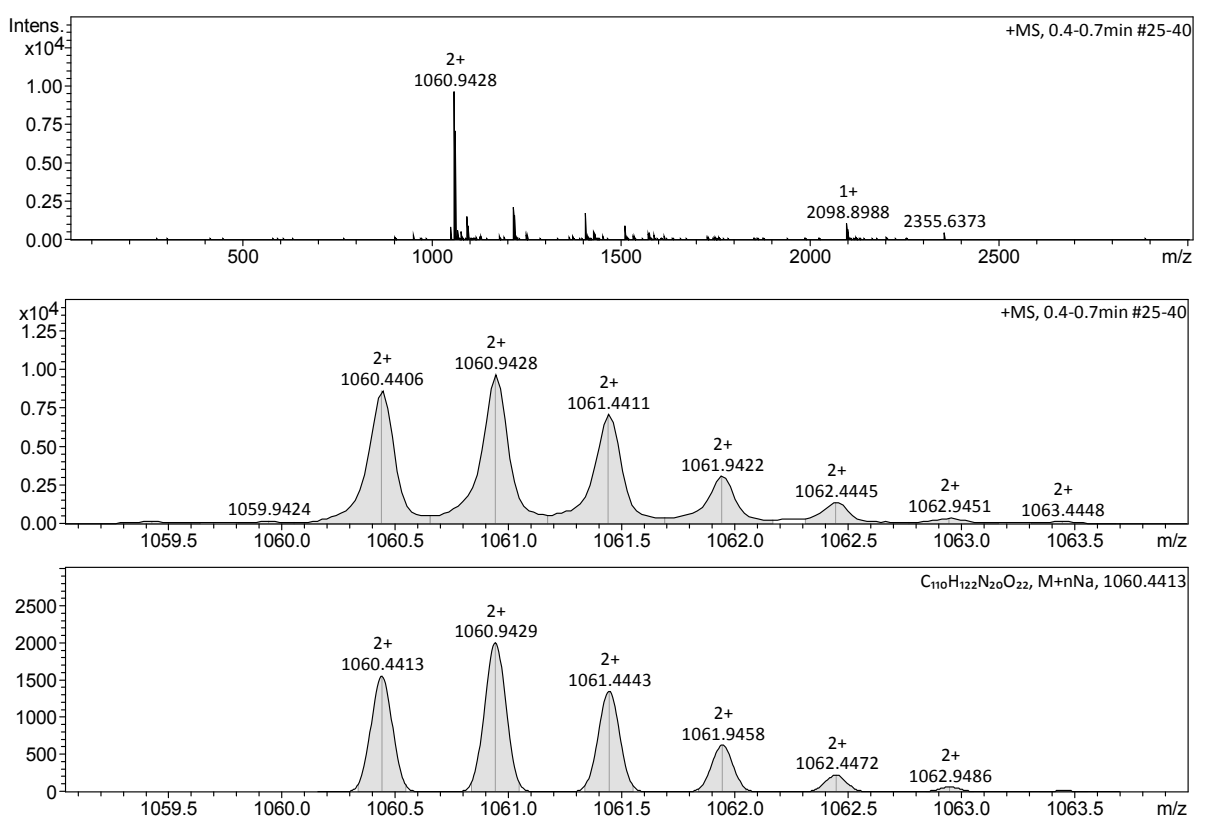

Figure S41. ESI-TOF MS spectra of MD $_{\text {amide }}[\mathbf{G}-1]$.
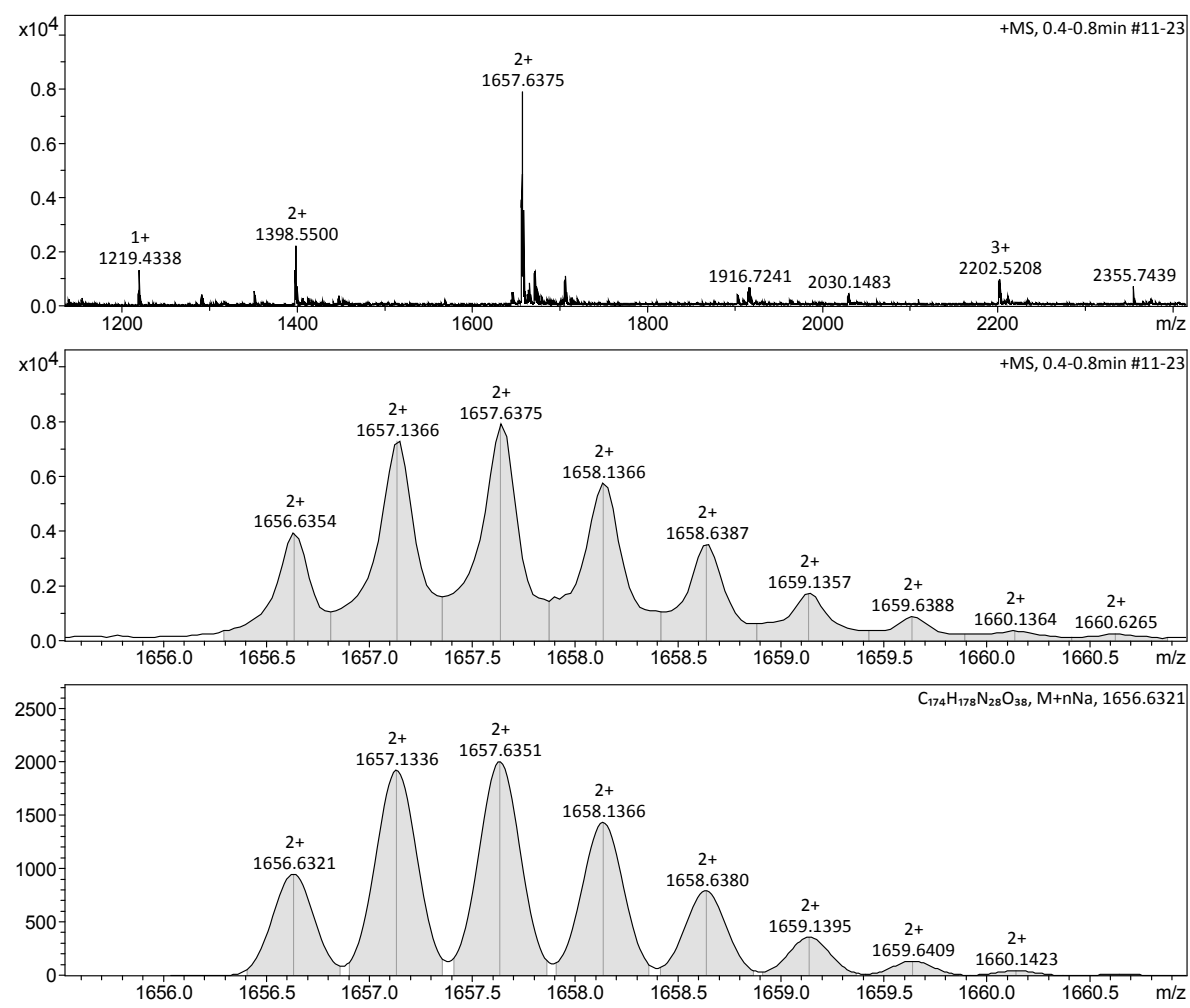

Figure S42. ESI-TOF MS spectra of $\mathbf{M D}_{\text {amide }}[\mathbf{G}-2]$. 

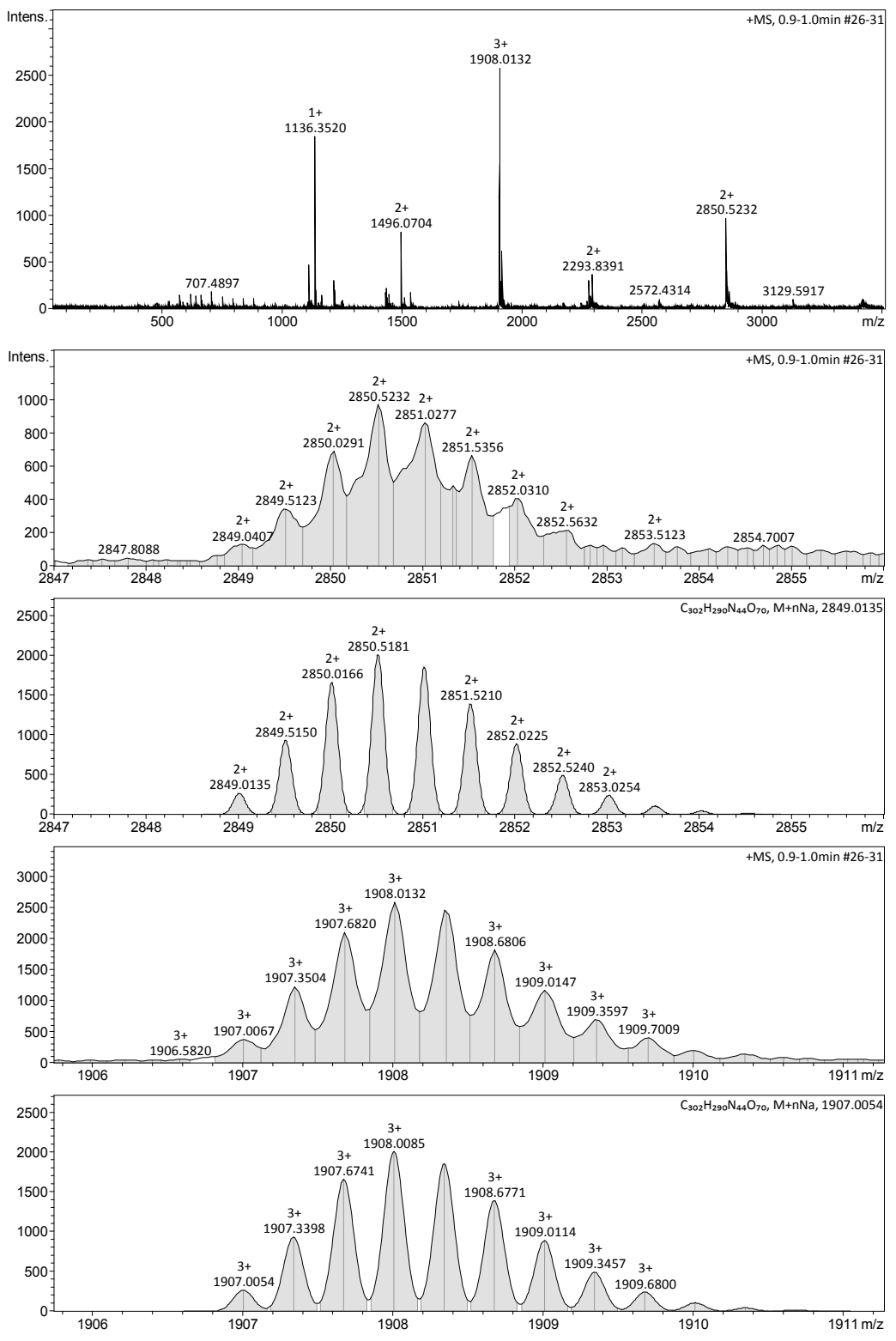

Figure S43. ESI-TOF MS spectra of $\mathbf{M D}_{\text {amide }}[\mathbf{G - 3}$. 
a)

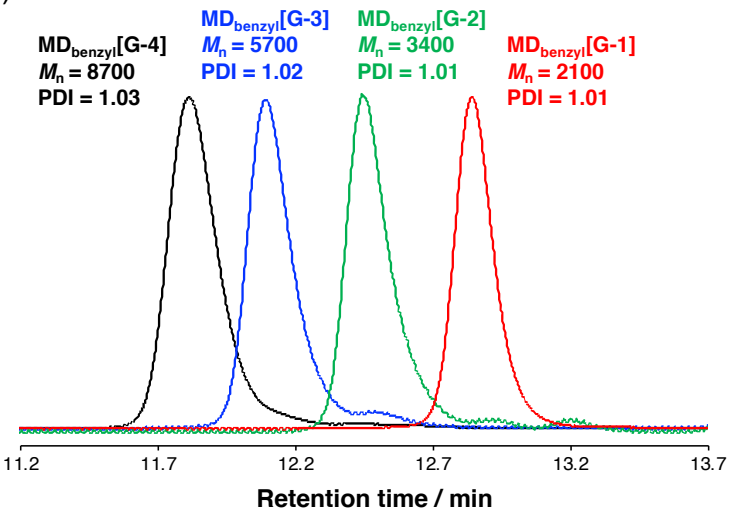

b)

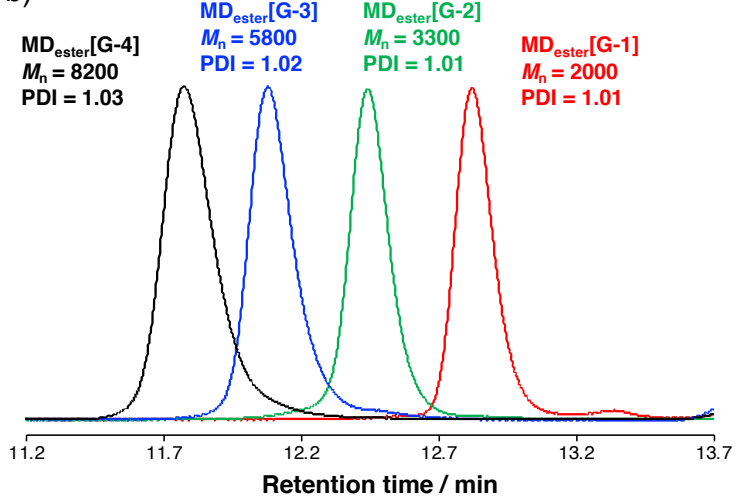

c)

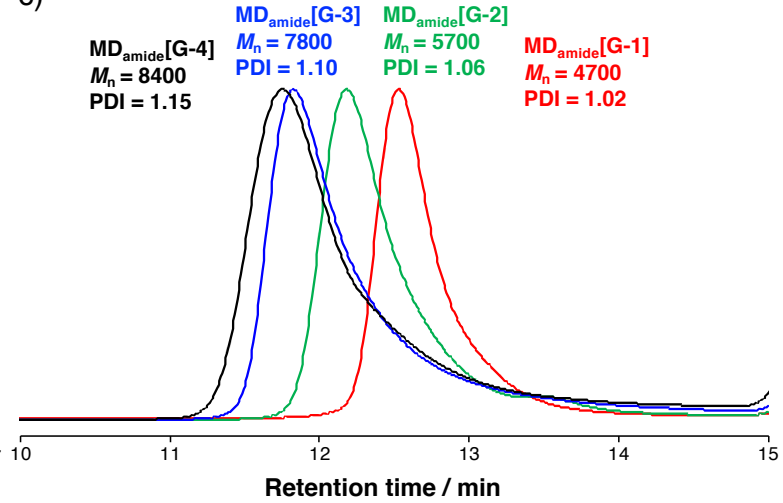

Figure S44. GPC profiles of a) MD $\mathbf{b e n z y l}_{\text {, b) }} \mathbf{M D}_{\text {ester }}$ eluted with THF and c) MD $\mathbf{M}_{\text {amide }}$ eluted with DMF. 


\subsection{Synthesis of MPS}

Scheme S1. Synthetic procedure for MPS.
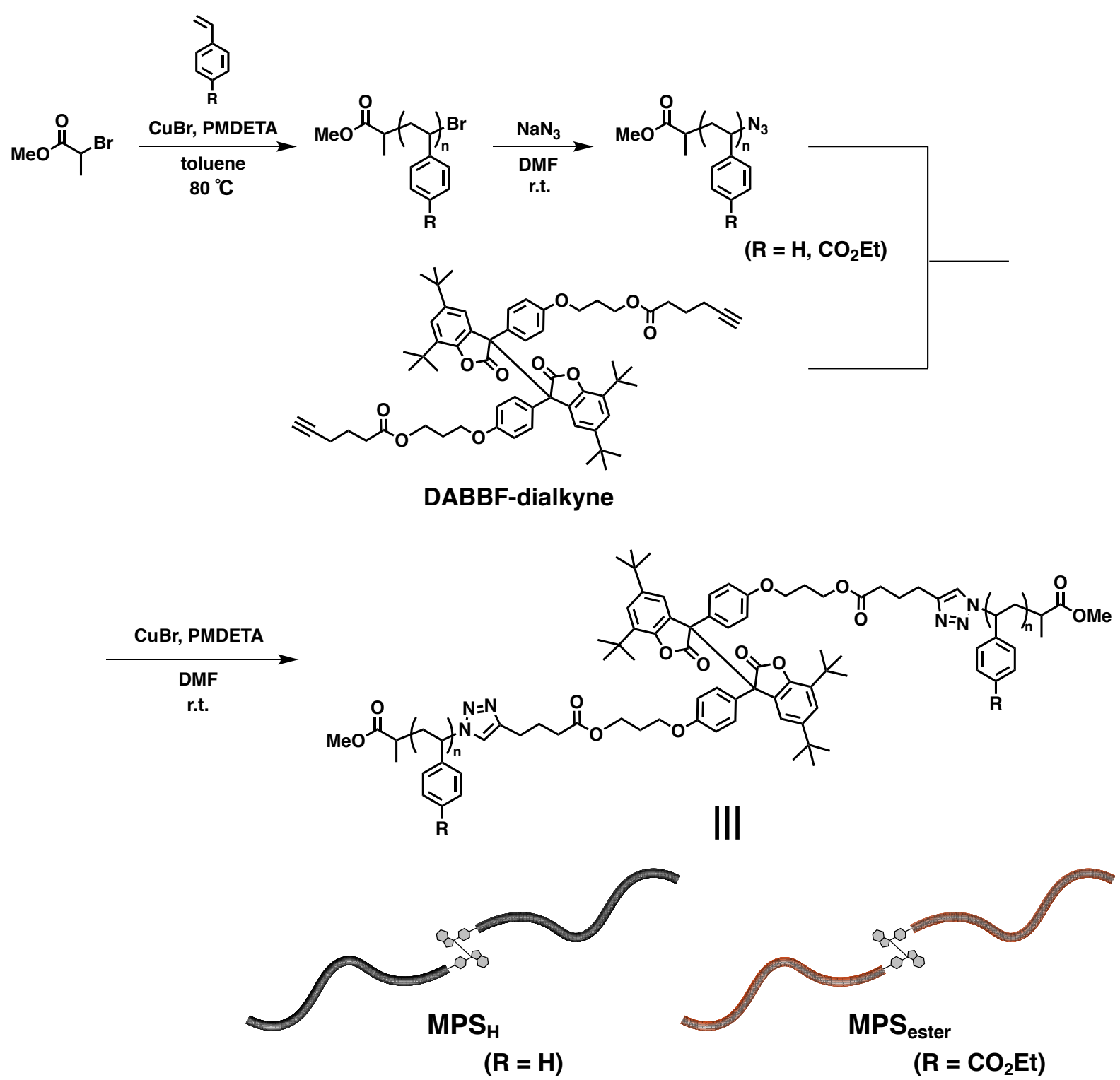


\section{Synthesis of MPS ${ }_{H}{ }^{1}$}

\section{$\mathrm{PS}-\mathrm{Br}$}

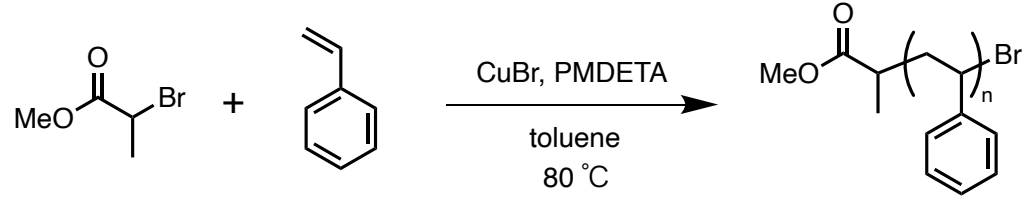

PS-Br

Under a nitrogen atmosphere, PMDETA $(1.09 \mathrm{~mL}, 5.22 \mathrm{mmol})$ was added to a solution of styrene $(72.0 \mathrm{~mL}$, $626 \mathrm{mmol})$, methyl 2-bromopropionate $(0.665 \mathrm{~mL}, 5.22 \mathrm{mmol})$, and $\mathrm{CuBr}(0.750 \mathrm{~g}, 5.23 \mathrm{mmol})$ in anhydrous toluene $(72 \mathrm{~mL})$ bubbled with nitrogen for 20 minutes. After being bubbled with nitrogen for another 20 minutes, the resulting solution was heated to $80{ }^{\circ} \mathrm{C}$ for $10 \mathrm{~h}$. After cooling to r.t., the reaction mixture was diluted with THF, then filtered through a column filled with neutral alumina in order to remove the copper complex. The resultant mixture was precipitated into methanol 3 times. Following filtration, the precipitate was dried in vacuo to afford the product as a white solid $(30.2 \mathrm{~g}, 96 \%)$. The $M_{\mathrm{n}}$ and $M_{\mathrm{w}} / M_{\mathrm{n}}$ values were determined by analytical GPC with polystyrene standards. $M_{\mathrm{n}}=6500 \mathrm{~g} \mathrm{~mol}^{-1}, M_{\mathrm{w}} / M_{\mathrm{n}}=1.10$.

${ }^{1} \mathrm{H}$ NMR (500 MHz, $\mathrm{CDCl}_{3}$ ): $\delta /$ ppm 7.30-6.30 (br, aromatic), 4.51-4.40 (br, $\left.\mathrm{CH}-\mathrm{Br}\right), 3.50-3.40\left(\mathrm{~m}, \mathrm{OCH}_{3}\right)$, 2.40-1.25 (br, CH, CH 2$), 0.97-0.86\left(\mathrm{~m}, \mathrm{CH}_{3}\right)$; FT-IR $\left(\mathrm{KBr}, \mathrm{cm}^{-1}\right): 3060,3026,2923,1736,1602,1493,1452$, $1029,756,698,540$

\section{PS-N 3}<smiles>COC(=O)C(C)C(C)(C)CC(Br)c1ccccc1</smiles>

$\mathrm{PS}-\mathrm{Br}$

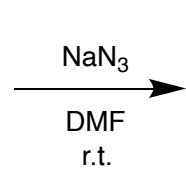

t.<smiles>COC(=O)C(C)C(C)(C)CC(N)c1ccccc1</smiles>

PS- $\mathrm{N}_{3}$

$\operatorname{PS}-\operatorname{Br}\left(M_{\mathrm{n}}=6500, \mathrm{PDI}=1.10,2.01 \mathrm{~g}, 0.309 \mathrm{mmol}\right)$ and sodium azide $(0.229 \mathrm{~g}, 3.52 \mathrm{mmol})$ in anhydrous DMF $(25 \mathrm{~mL})$ were stirred at r.t. for 1 day. The reaction mixture was precipitated into water. Following filtration, the product was dried in vacuo to afford PS-N $\mathbf{3}$ as a white solid (1.94 g, quant.). The $M_{\mathrm{n}}$ and $M_{\mathrm{w}} / M_{\mathrm{n}}$ values were determined by analytical GPC with polystyrene standards. $M_{\mathrm{n}}=5900 \mathrm{~g} \mathrm{~mol}^{-1}, M_{\mathrm{w}} / M_{\mathrm{n}}=1.08$. ${ }^{1} \mathrm{H}$ NMR (500 MHz, $\mathrm{CDCl}_{3}$ ): $\delta /$ ppm 7.29-6.30 (br, aromatic), 4.02-3.85 (br, $\left.\mathrm{CH}-\mathrm{N}_{3}\right), 3.50-3.40\left(\mathrm{~m}, \mathrm{OCH}_{3}\right)$, 2.39-1.18 (br, backbone), 1.00-0.86 (m, $\left.\mathrm{CH}_{3}\right)$; FT-IR (KBr, $\left.\mathrm{cm}^{-1}\right): 3060,3026,2923,2093,1736,1602,1493$, 1452, 1071, 756, 698. 
MPS $_{H}$
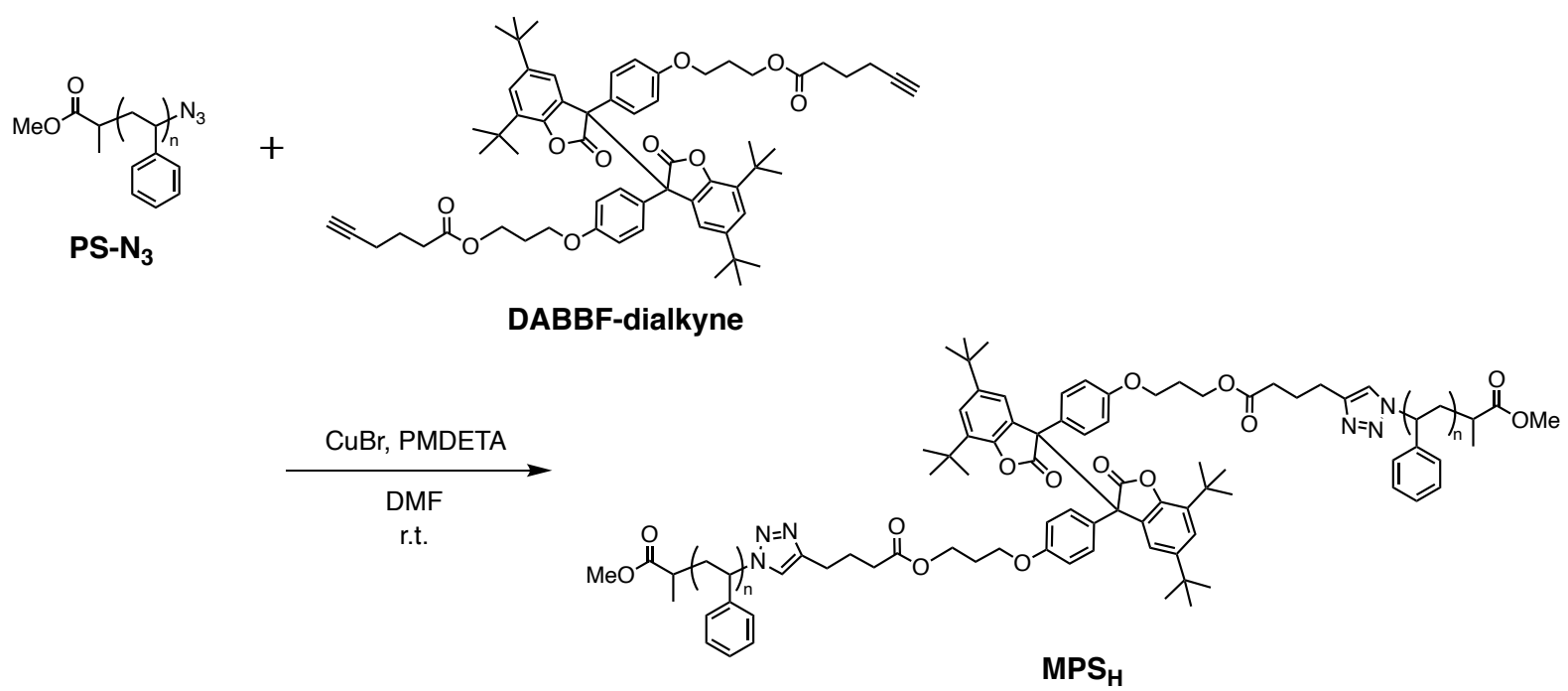

Under a nitrogen atmosphere, PMDETA $(0.193 \mathrm{~mL}, 0.924 \mathrm{mmol})$ was added to a solution of PS-N $\mathbf{N}_{\mathbf{3}}\left(M_{\mathrm{n}}=\right.$ 5900, PDI = 1.08, $1.93 \mathrm{~g}, 0.327 \mathrm{mmol})$, DABBF-dialkyne (0.149 g, $0.152 \mathrm{mmol}), \mathrm{CuBr}(68.4 \mathrm{mg}, 0.477$ $\mathrm{mmol})$, and $\mathrm{Cu}(27.2 \mathrm{mg}, 0.428 \mathrm{mmol})$ in anhydrous DMF (40 mL) bubbled with nitrogen for 20 minutes. After being bubbled with nitrogen for another 20 minutes, the resulting solution was allowed to stir at r.t. for $5 \mathrm{~h}$. The reaction mixture was diluted with THF and filtered through a column filled with neutral alumina in order to remove the copper complex. Following evaporation, the resultant mixture was poured into methanol. The crude product was subsequently purified by fractional precipitation 5 times with chloroform and hexane. After precipitation into methanol, the product was dried in vacuo to afford $\mathbf{M P S}_{\mathbf{H}}$ as a white solid $(0.934 \mathrm{~g}$, $41 \%$ ). The $M_{\mathrm{n}}$ and $M_{\mathrm{w}} / M_{\mathrm{n}}$ values were determined by both ${ }^{1} \mathrm{H}$ NMR and analytical GPC with polystyrene standards. $M_{\mathrm{n}}$ calculated by ${ }^{1} \mathrm{H}$ NMR was $M_{\mathrm{n}, \mathrm{NMR}}=15700 \mathrm{~g} \mathrm{~mol}^{-1}$, and by GPC was $M_{\mathrm{n}, \mathrm{GPC}}=14900 \mathrm{~g} \mathrm{~mol}^{-}$ ${ }^{1}, M_{\mathrm{w}} / M_{\mathrm{n}}=1.06$.

${ }^{1} \mathrm{H}$ NMR (500 MHz, $\mathrm{CDCl}_{3}$ ): $\delta / \mathrm{ppm} 7.30-6.30$ (br, aromatic), 5.08-4.92 (br, $\left.\mathrm{CH}\right), 4.25-4.20\left(\mathrm{~m}, \mathrm{CH}_{2}\right), 4.03-$ $4.00\left(\mathrm{~m}, \mathrm{CH}_{2}\right), 3.50-3.40\left(\mathrm{~m}, \mathrm{OCH}_{3}\right), 2.67-0.87$ (m, backbone, $\left.\mathrm{CH}_{2}, \mathrm{CH}_{3}\right)$; FT-IR $\left(\mathrm{KBr}, \mathrm{cm}^{-1}\right): 3083,3060$, 3026, 2923, 2849, 1944, 1795, 1736, 1602, 1493, 1452, 1364, 1182, 1086, 1029, 906, 756, 698, 540. 


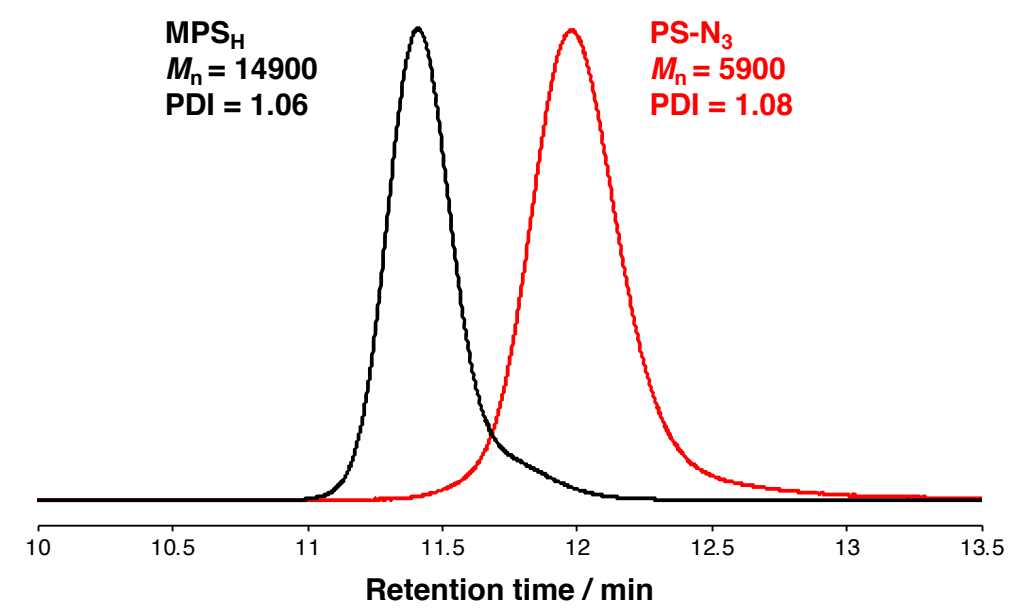

Figure S45. GPC profiles of MPS $_{\mathbf{H}}$ (black) and PS-N $\mathbf{3}$ (red).

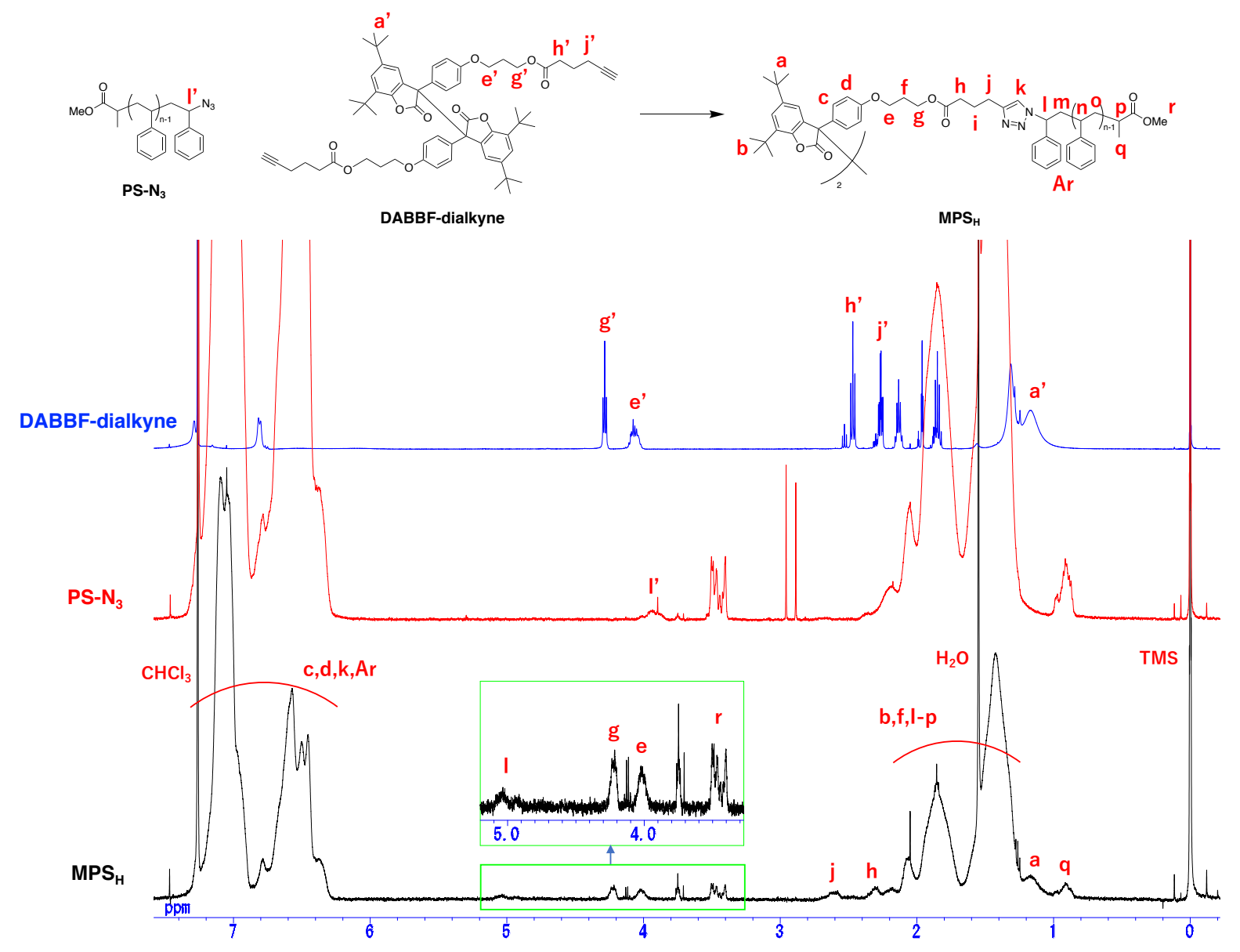

Figure S46. ${ }^{1} \mathrm{H}$ NMR spectra of MPS $_{\mathbf{H}}$ (black), PS-N $\mathbf{3}$ (red), and DABBF-dialkyne (blue) (500 MHz, $\left.\mathrm{CDCl}_{3}\right)$. 


\section{Synthesis of MPS ester}

\section{4-Ethoxycarbonylstyrene ${ }^{4}$}

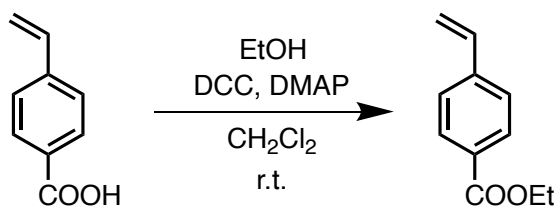

To a solution of 4-vinylbenzoic acid (16.3 g, $0.110 \mathrm{~mol}), N, N^{\prime}$-dicyclohexylcarbodiimide (DCC) $(27.2 \mathrm{~g}$, $0.132 \mathrm{~mol})$, and $N, N^{\prime}$-dimethyl-4-aminopyridine (DMAP) in anhydrous dichloromethane $(800 \mathrm{~mL})$ was added ethanol $(64.0 \mathrm{~mL}, 1.10 \mathrm{~mol})$ and the mixture was stirred at r.t. for $2 \mathrm{~h}$. After evaporation, a white precipitate was removed by filtration. The crude filtrate was subsequently purified by flash chromatography (ethyl acetate/hexane $=1: 10)$, affording colorless liquid $(16.2 \mathrm{~g}, 84 \%)$.

${ }^{1} \mathrm{H}$ NMR (500 MHz, $\left.\mathrm{CDCl}_{3}\right): \delta / \mathrm{ppm} 8.01(\mathrm{~d}, J=8.3 \mathrm{~Hz}, 2 \mathrm{H}$, aromatic), 7.46 (d, $J=8.3 \mathrm{~Hz}, 2 \mathrm{H}$, aromatic), $6.76(\mathrm{dd}, J=10.9 \mathrm{~Hz}, 17.6 \mathrm{~Hz}, 2 \mathrm{H}, \mathrm{CH}), 5.87\left(\mathrm{~d}, J=17.6 \mathrm{~Hz}, 1 \mathrm{H}, \mathrm{CH}_{2}\right), 5.38\left(\mathrm{~d}, J=10.9 \mathrm{~Hz}, 2 \mathrm{H}, \mathrm{CH}_{2}\right)$, $4.38\left(\mathrm{q}, J=7.1 \mathrm{~Hz}, 2 \mathrm{H}, \mathrm{CH}_{2}\right), 1.40\left(\mathrm{t}, J=7.1 \mathrm{~Hz}, 3 \mathrm{H}, \mathrm{CH}_{3}\right)$

\section{PSester-Br}

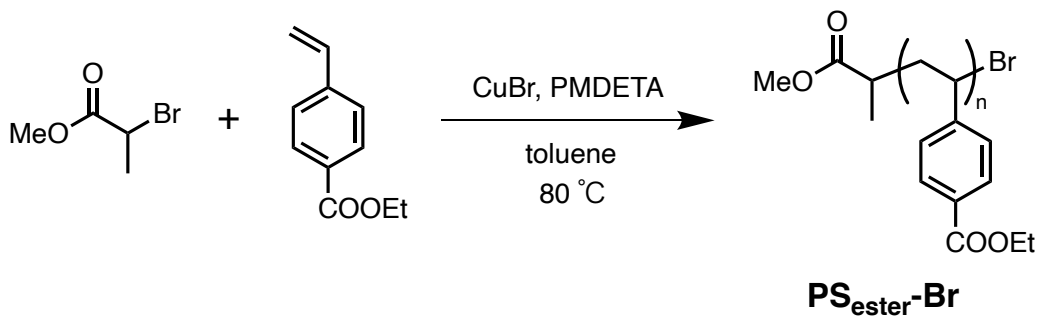

Under a nitrogen atmosphere, PMDETA $(50.0 \mu \mathrm{L}, 0.243 \mathrm{mmol})$ was added to a solution of 4ethoxycarbonylstyrene (3.01 g, $17.1 \mathrm{mmol})$, methyl 2-bromopropionate $(50.0 \mu \mathrm{L}, 0.449 \mathrm{mmol})$, and $\mathrm{CuBr}$ (35.4 mg, $0.247 \mathrm{mmol})$ in anhydrous toluene $(3.0 \mathrm{~mL}$ ) bubbled with nitrogen for 20 minutes. After being bubbled with nitrogen for another 20 minutes, the resulting solution was heated to $80{ }^{\circ} \mathrm{C}$ for $3 \mathrm{~h}$. After cooling to r.t., the reaction mixture was diluted with THF, then filtered through a column filled with neutral alumina in order to remove the copper complex. The resultant mixture was concentrated and precipitated into methanol. Following filtration, the precipitate was dried in vacuo to afford the product as a white solid (2.07 g, 77\%). The $M_{\mathrm{n}}$ and $M_{\mathrm{w}} / M_{\mathrm{n}}$ values were determined by both ${ }^{1} \mathrm{H}$ NMR and analytical GPC with polystyrene standards. $M_{\mathrm{n}}$ calculated by ${ }^{1} \mathrm{H}$ NMR was $M_{\mathrm{n}, \mathrm{NMR}}=6000 \mathrm{~g} \mathrm{~mol}^{-1}$, and by GPC was $M_{\mathrm{n}, \mathrm{GPC}}=$ $5500 \mathrm{~g} \mathrm{~mol}^{-1}, M_{\mathrm{w}} / M_{\mathrm{n}}=1.07$.

${ }^{1} \mathrm{H}$ NMR (500 MHz, $\mathrm{CDCl}_{3}$ ): $\delta / \mathrm{ppm}$ 8.03-7.44 (br, aromatic), 7.20-6.30 (br, aromatic), 4.36 (br, $\mathrm{CH}_{2}, \mathrm{CH}-$ $\mathrm{Br}), 3.50-3.36\left(\mathrm{~m}, \mathrm{OCH}_{3}\right), 2.38-1.12$ (br, backbone), 1.39 (br, $\left.\mathrm{CH}_{3}\right)$, 0.97-0.83 (m, $\left.\mathrm{CH}_{3}\right)$; FT-IR $\left(\mathrm{KBr}, \mathrm{cm}^{-1}\right)$ : $2928,1718,1609,1420,1366,1278,1179,1104,1021,854,773,709,478$. 


\section{$\mathrm{PS}_{\text {ester-N}} \mathrm{N}_{3}$}

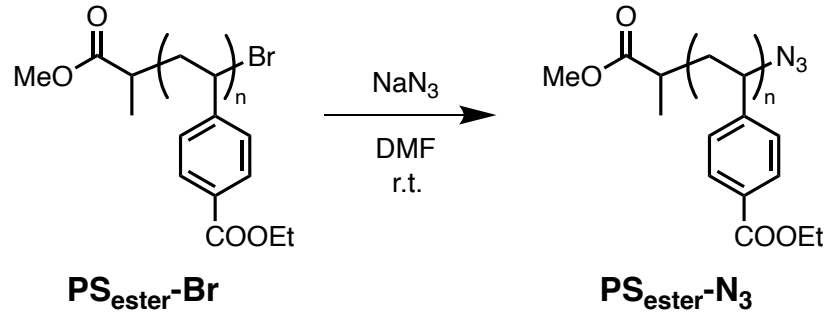

$\mathbf{P S}_{\text {ester- }} \mathbf{B r}\left(M_{\mathrm{n}, \mathrm{NMR}}=6000, \mathrm{PDI}=1.07,2.07 \mathrm{~g}, 0.344 \mathrm{mmol}\right)$ and sodium azide $(0.337 \mathrm{~g}, 5.18 \mathrm{mmol})$ in anhydrous DMF $(20 \mathrm{~mL})$ were stirred at r.t. for 1 day. The reaction mixture was precipitated into water. Following filtration, the product was dried in vacuo to afford $\mathbf{P S} \mathbf{S}_{\text {ester }}-\mathbf{N}_{3}$ as a white solid ( $2.00 \mathrm{~g}$, quant.). The $M_{\mathrm{n}}$ and $M_{\mathrm{w}} / M_{\mathrm{n}}$ values were determined by both ${ }^{1} \mathrm{H}$ NMR and analytical GPC with polystyrene standards. $M_{\mathrm{n}}$ calculated by ${ }^{1} \mathrm{H}$ NMR was $M_{\mathrm{n}, \mathrm{NMR}}=5800 \mathrm{~g} \mathrm{~mol}^{-1}$, and by GPC was $M_{\mathrm{n}, \mathrm{GPC}}=5400 \mathrm{~g} \mathrm{~mol}^{-1}, M_{\mathrm{w}} / M_{\mathrm{n}}=1.08$. ${ }^{1} \mathrm{H}$ NMR (500 MHz, $\mathrm{CDCl}_{3}$ ): $\delta / \mathrm{ppm}$ 7.97-7.45 (br, aromatic), 7.12-6.28 (br, aromatic), 4.37 (br, $\mathrm{CH}_{2}$ ), 4.023.77 (m, CH-N $)_{3}$, 3.49-3.37 (m, $\left.\mathrm{OCH}_{3}\right), 2.36-1.11$ (br, backbone), 1.39 (br, $\left.\mathrm{CH}_{3}\right), 0.95-0.84\left(\mathrm{~m}, \mathrm{CH}_{3}\right)$; FTIR (KBr, $\left.\mathrm{cm}^{-1}\right): 2981,2931,2098,1717,1609,1419,1367,1310,1278,1180,1104,1021,854,773,709$.

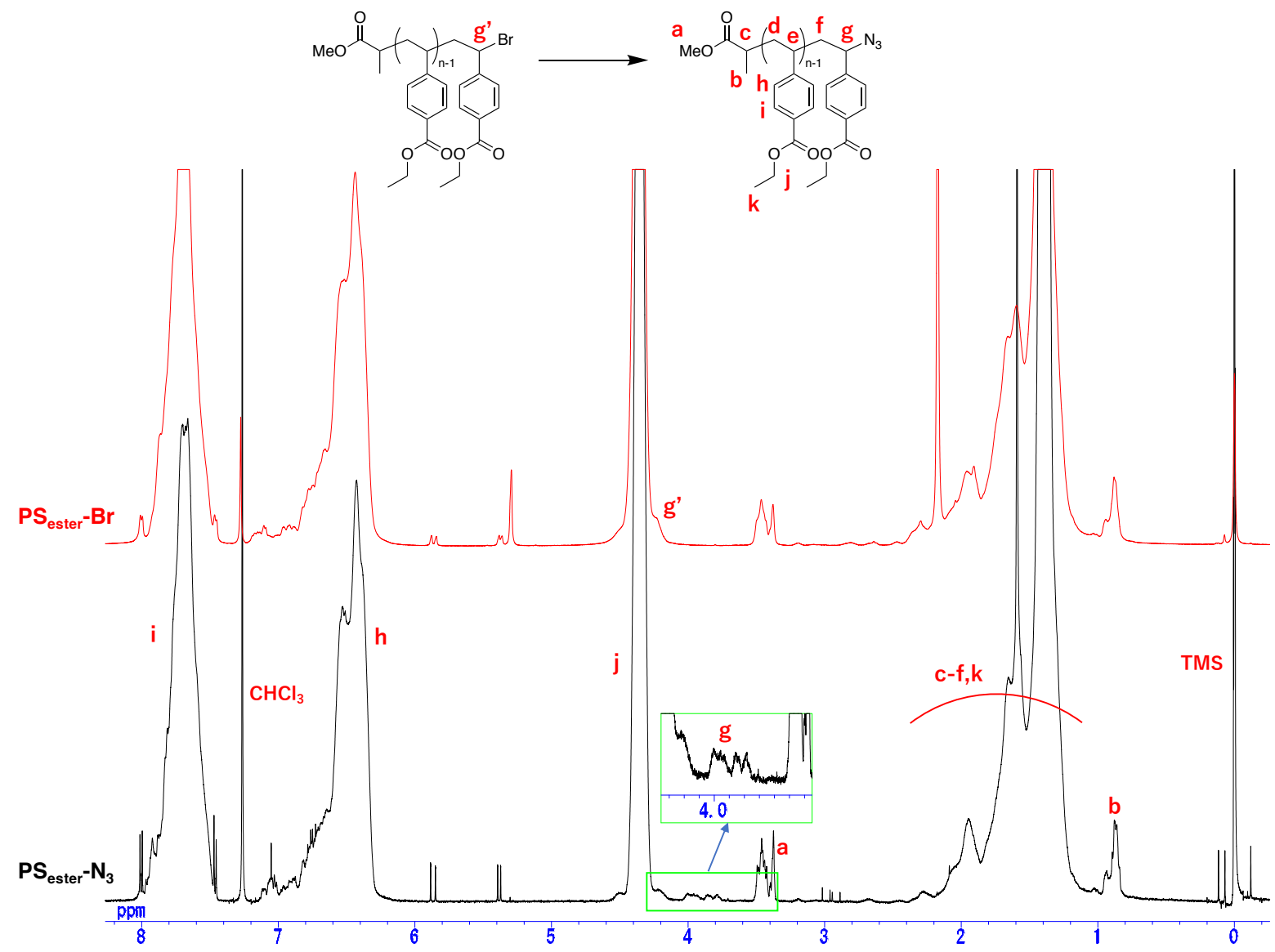

Figure S47. ${ }^{1} \mathrm{H}$ NMR spectra of $\mathbf{P S} \mathbf{S}_{\text {ester- }} \mathbf{N}_{\mathbf{3}}$ (black) and $\mathbf{P S}$ ester-Br (red) $\left(500 \mathrm{MHz}, \mathrm{CDCl}_{3}\right.$ ). 


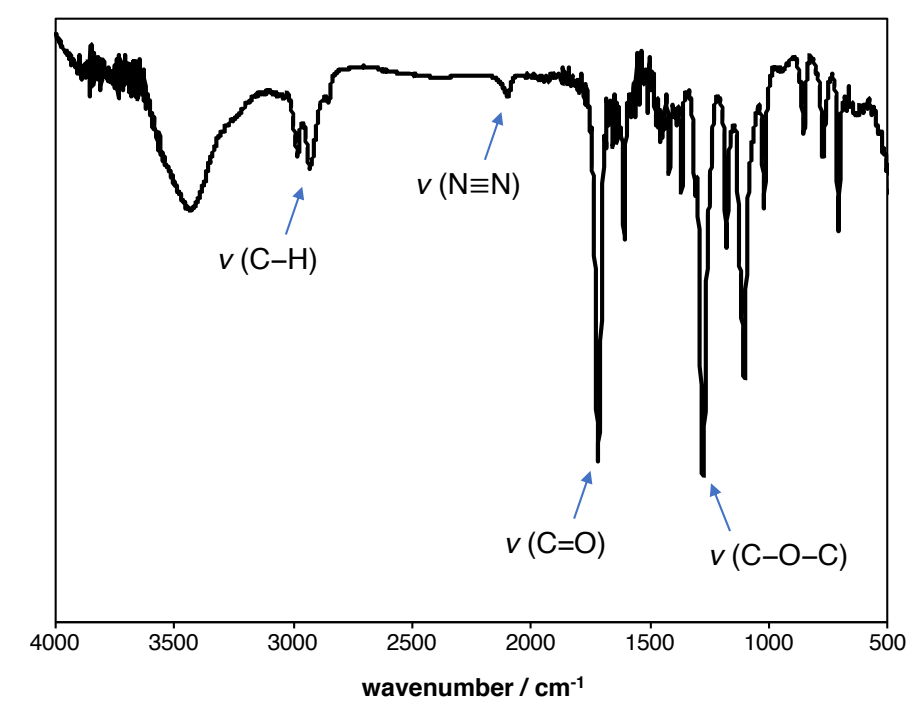

Figure S48. IR spectrum of $\mathbf{P S}_{\text {ester- }} \mathbf{N}_{\mathbf{3}}(\mathrm{KBr})$.

\section{MPS ester}

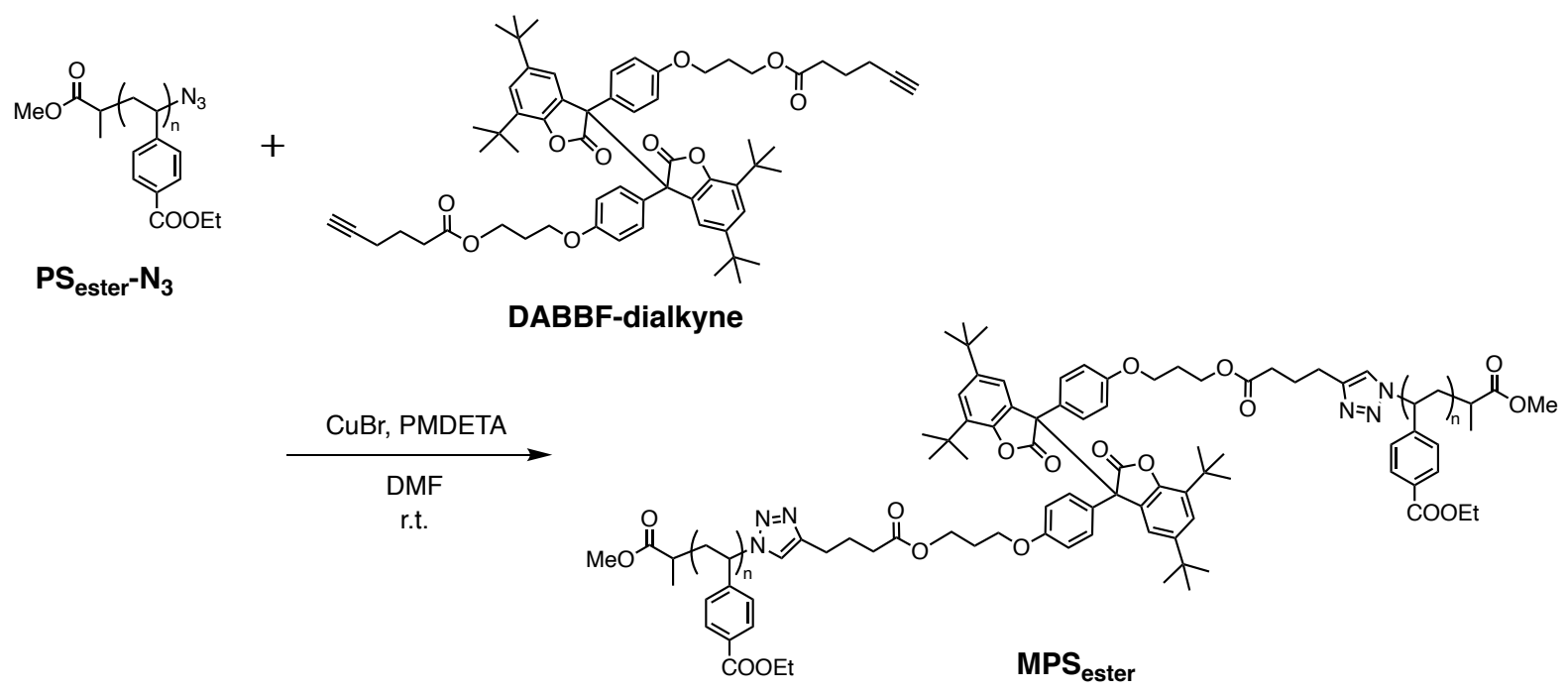

Under a nitrogen atmosphere, PMDETA $(0.155 \mathrm{~mL}, 0.745 \mathrm{mmol})$ was added to a solution of $\mathbf{P S}$ ester- $\mathbf{N}_{\mathbf{3}}\left(M_{\mathrm{n}}\right.$, $\mathrm{NMR}=5800, \mathrm{PDI}=1.08,1.91 \mathrm{~g}, 0.329 \mathrm{mmol})$, DABBF-dialkyne (0.146 g, $0.149 \mathrm{mmol}), \mathrm{CuBr}(64.7 \mathrm{mg}$, $0.451 \mathrm{mmol})$, and $\mathrm{Cu}(19.7 \mathrm{mg}, 0.310 \mathrm{mmol})$ in anhydrous DMF $(30 \mathrm{~mL})$ bubbled with nitrogen for 20 minutes. After being bubbled with nitrogen for another 20 minutes, the resulting solution was allowed to stir at r.t. for $1.5 \mathrm{~h}$. The reaction mixture was diluted with THF and filtered through a column filled with neutral alumina in order to remove the copper complex. Following evaporation, the resultant mixture was poured into water. The precipitate was dissolved in chloroform and dried over anhydrous $\mathrm{MgSO}_{4}$, filtered, and concentrated. The crude product was subsequently purified by fractional precipitation 7 times with chloroform and hexane. After precipitation into methanol, the product was dried in vacuo at $40^{\circ} \mathrm{C}$ overnight to afford MPS ester as a white solid $(0.949 \mathrm{~g}, 50 \%)$. The $M_{\mathrm{n}}$ and $M_{\mathrm{w}} / M_{\mathrm{n}}$ values were determined by both ${ }^{1} \mathrm{H}$ NMR and analytical GPC with polystyrene standards. $M_{\mathrm{n}}$ calculated by ${ }^{1} \mathrm{H}$ NMR was $M_{\mathrm{n}, \mathrm{NMR}}=14300 \mathrm{~g}$ 
mol $^{-1}$, and by GPC was $M_{\mathrm{n}, \mathrm{GPC}}=11900 \mathrm{~g} \mathrm{~mol}^{-1}, M_{\mathrm{w}} / M_{\mathrm{n}}=1.06$.

${ }^{1} \mathrm{H}$ NMR (500 MHz, $\mathrm{CDCl}_{3}$ ): $\delta / \mathrm{ppm} 7.91-7.47$ (br, aromatic), 7.08-6.30 (br, aromatic), 5.08-4.83 (br, $\mathrm{CH}$ ), 4.35 (br, $\mathrm{CH}_{2}$ ), 4.19 (br, $\mathrm{CH}_{2}$ ), 4.01 (br, $\left.\mathrm{CH}_{2}\right), 3.49-3.37\left(\mathrm{~m}, \mathrm{OCH}_{3}\right), 2.67-2.53\left(\mathrm{~m}, \mathrm{CH}_{2}\right), 2.33-2.23\left(\mathrm{~m}, \mathrm{CH}_{2}\right)$, 2.13-0.83 (br, backbone, $\mathrm{CH}_{2}, \mathrm{CH}_{3}$ ), 1.39 (br, $\mathrm{CH}_{3}$ ); FT-IR ( $\mathrm{KBr}, \mathrm{cm}^{-1}$ ): 2980, 2931, 1717, 1609, 1575, 1508 , $1447,1419,1392,1367,1277,1179,1103,1020,853,772,708$.

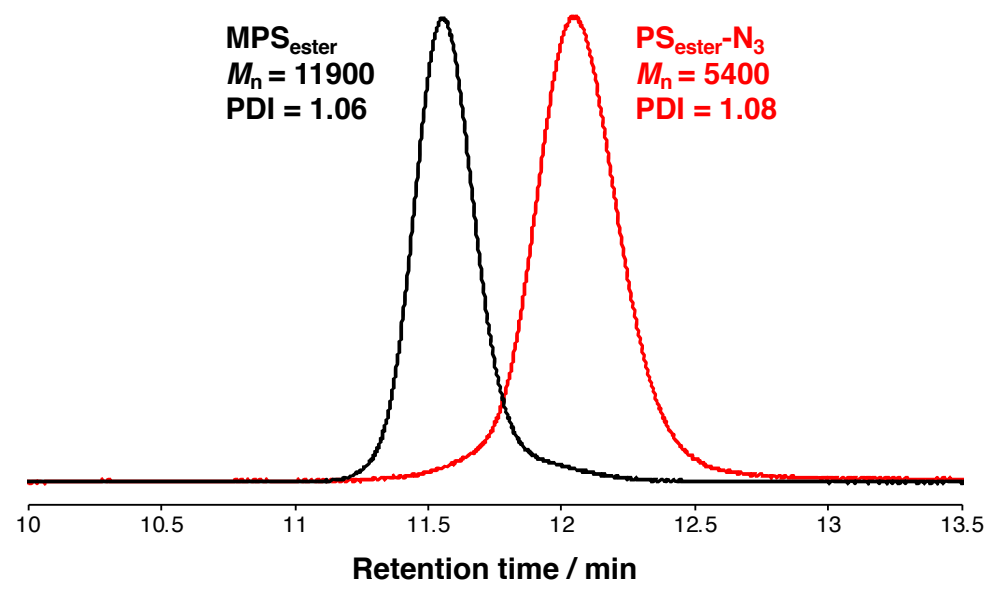

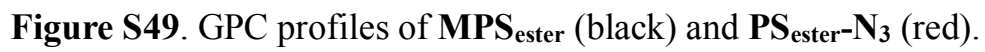




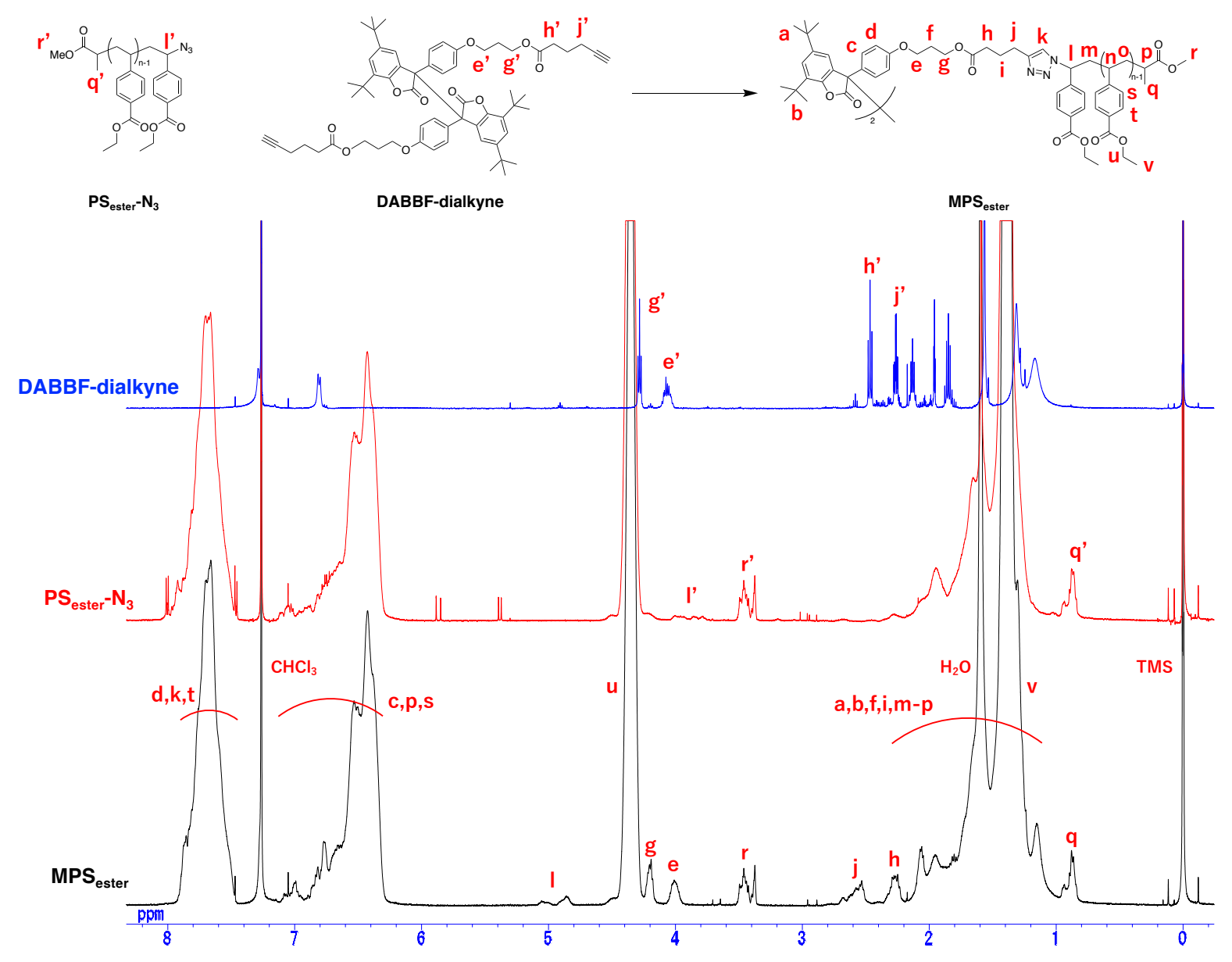

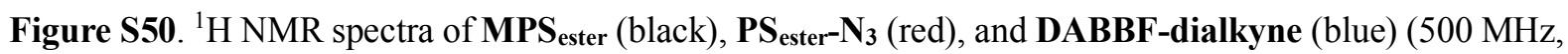
$\left.\mathrm{CDCl}_{3}\right)$.

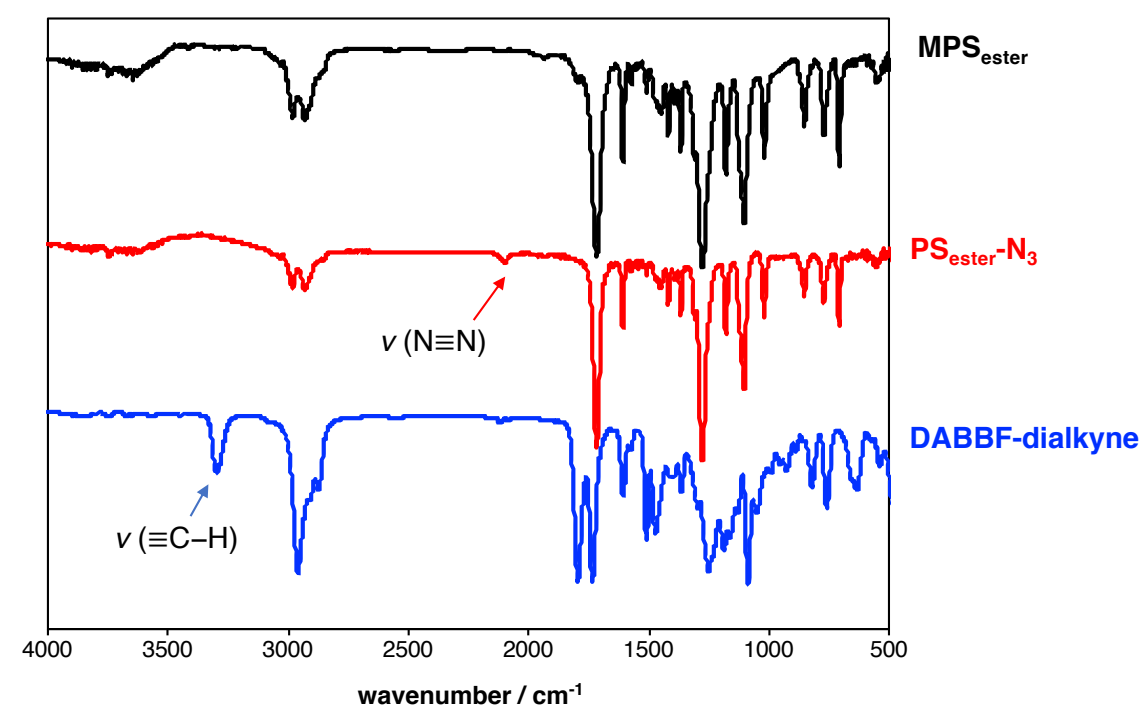

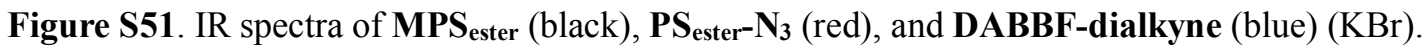




\section{Mechanical activation of MD and MPS}

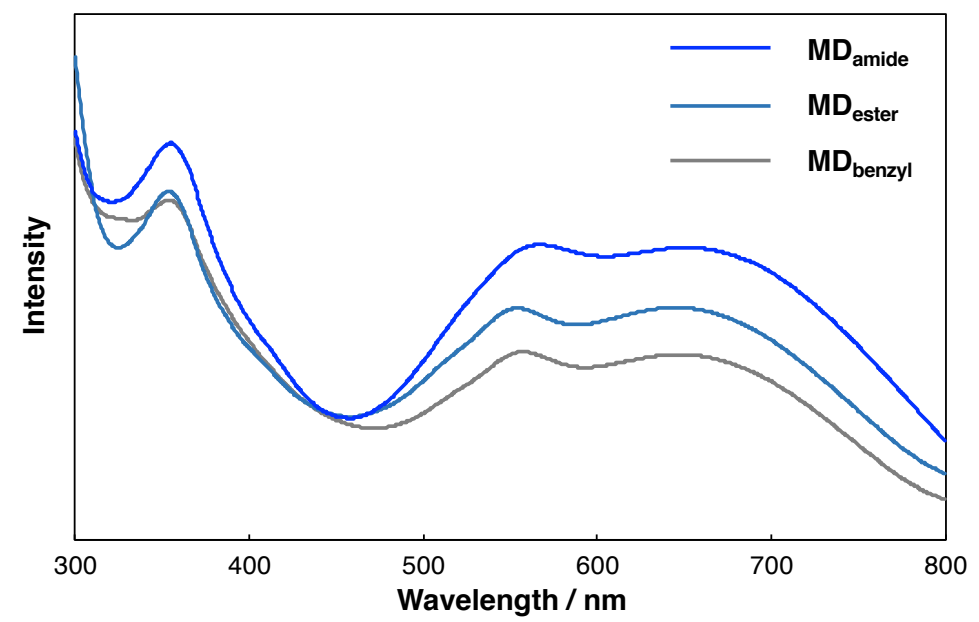

Figure S52. Solid-state UV-vis spectra of ground $3^{\text {rd }}$ generation MDs with distinct surfaces.

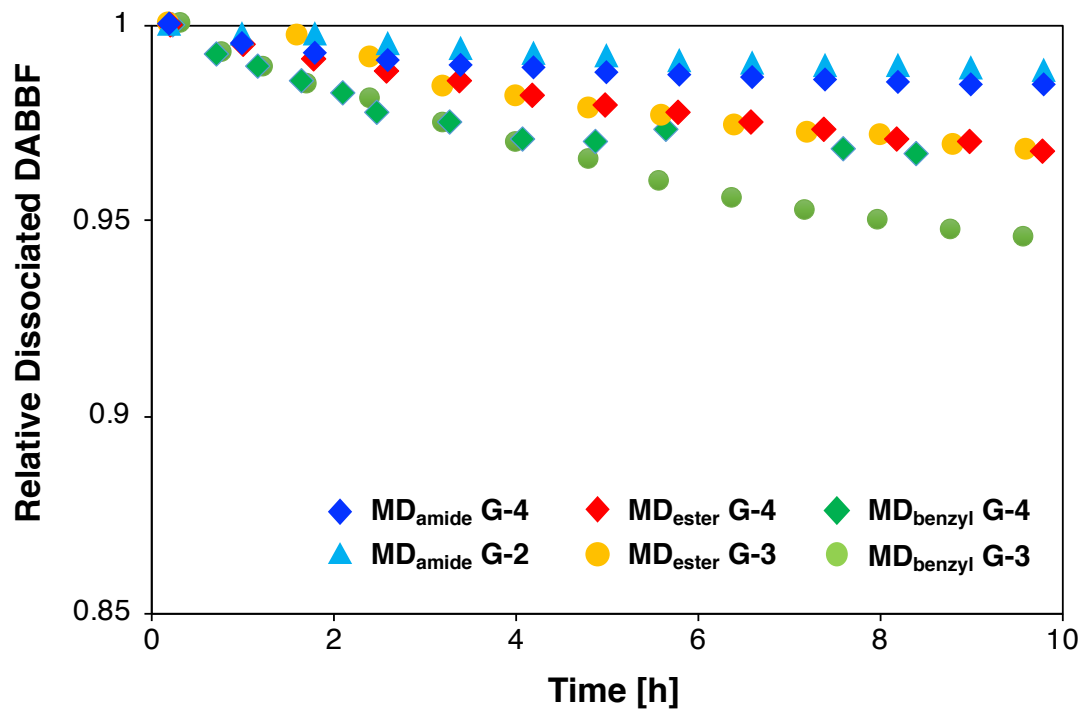

Figure S53. Radical attenuation behavior of MDs. 
a)
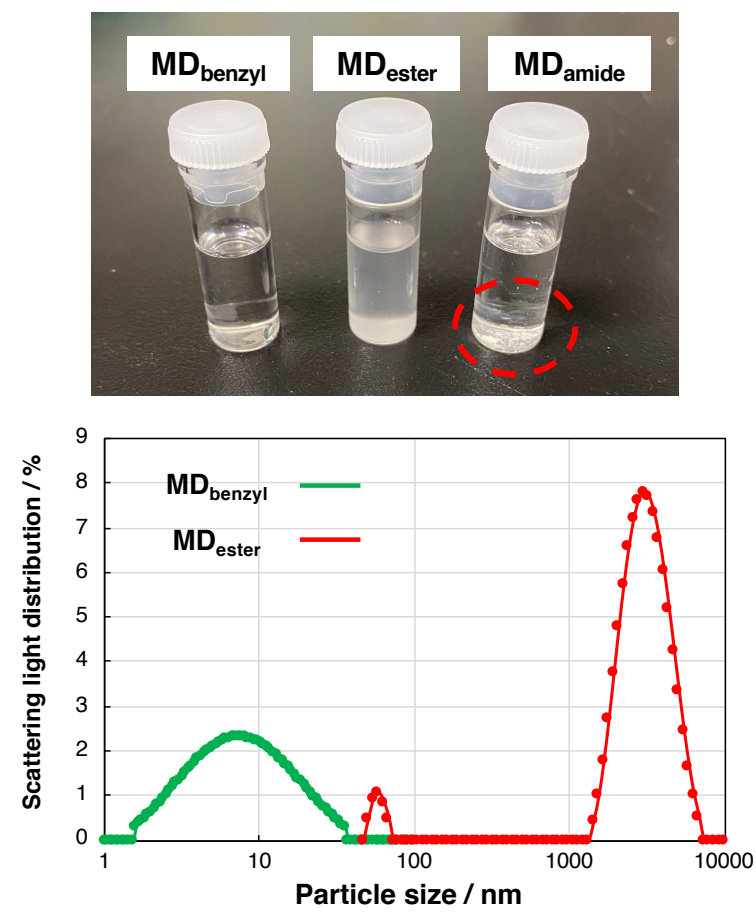

b)
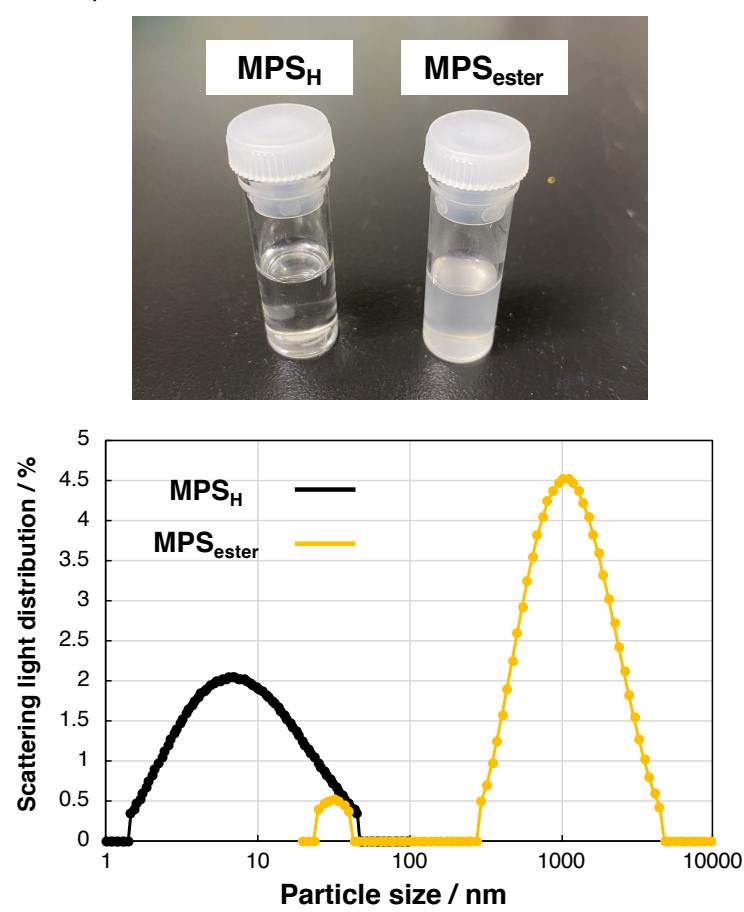

Figure S54. Solubility test (upper) and size distribution of polymeric nanoparticles by a DLS method (lower) for a) $4^{\text {th }}$ generation MDs and b) MPSs. Photographs and DLS measurements were taken 10 minutes after membrane filtration. MDs were dissolved in dioxane/cyclohexane $=1: 3\left(0.75 \mathrm{mg} \mathrm{mL}^{-1}\right)$, although MD $_{\text {amide }}$ [G-4] was completely insoluble in the mixed solvent. MPSs were dissolved in chloroform/cyclohexane $=1: 19\left(0.75 \mathrm{mg} \mathrm{mL}^{-1}\right)$. 
Table S1. $T_{\mathrm{g}}$ of MD and MPS together with the molecular weight and dissociation ratio of DABBF.

\begin{tabular}{|c|c|c|c|}
\hline & $T_{\mathrm{g}}\left[{ }^{\circ} \mathrm{C}\right]$ & $M\left[\mathrm{~g} \mathrm{~mol}^{-1}\right]$ & Dissociation ratio [\%] \\
\hline$M D_{\text {benzy }}[G-1]$ & 43 & 2581 & 0.0660 \\
\hline$M D_{\text {benzyl }}[G-2]$ & 44 & 4279 & 0.157 \\
\hline $\mathrm{MD}_{\text {benzyl }}[\mathrm{G}-3]$ & 44 & 7675 & 0.517 \\
\hline$M D_{\text {benzyl }}[G-4]$ & 46 & 14467 & 1.53 \\
\hline$M D_{\text {ester }}[G-1]$ & 48 & 2309 & 0.153 \\
\hline MD $D_{\text {ester }}[\mathrm{G}-2]$ & 58 & 3734 & 0.454 \\
\hline MD $D_{\text {ester }}[\mathrm{G}-3]$ & 60 & 6585 & 1.29 \\
\hline $\mathrm{MD}_{\text {ester }}[\mathrm{G}-4]$ & 61 & 12286 & 4.96 \\
\hline $\mathrm{MD}_{\text {amide }}[\mathrm{G}-1]$ & 116 & 2076 & 1.57 \\
\hline $\mathrm{MD}_{\text {amide }}[\mathrm{G}-2]$ & 142 & 3270 & 3.09 \\
\hline $\mathrm{MD}_{\text {amide }}[\mathrm{G}-3]$ & 159 & 5656 & 8.51 \\
\hline $\mathrm{MD}_{\text {amide }}[\mathrm{G}-4]$ & 162 & 10429 & 14.7 \\
\hline $\mathrm{MPS}_{\mathrm{H}}$ & 97 & 15700 & 3.72 \\
\hline MPS $_{\text {ester }}$ & 101 & 14300 & 4.90 \\
\hline
\end{tabular}

a)

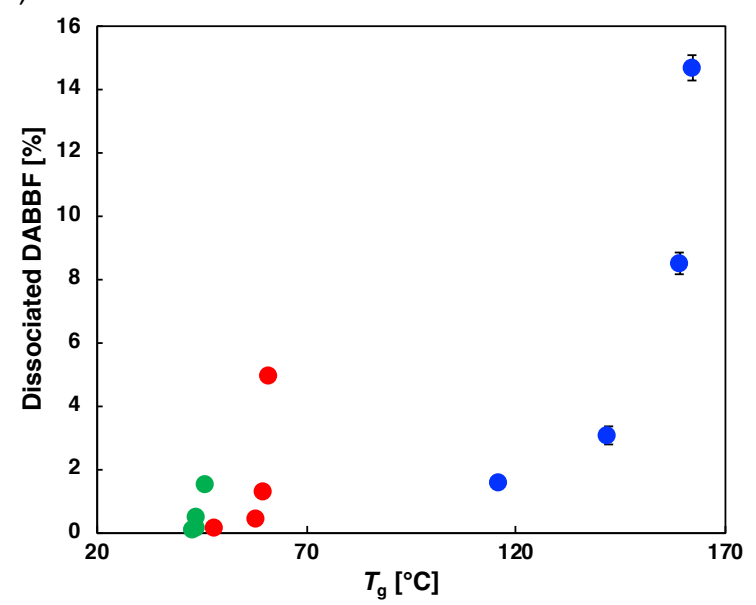

b)

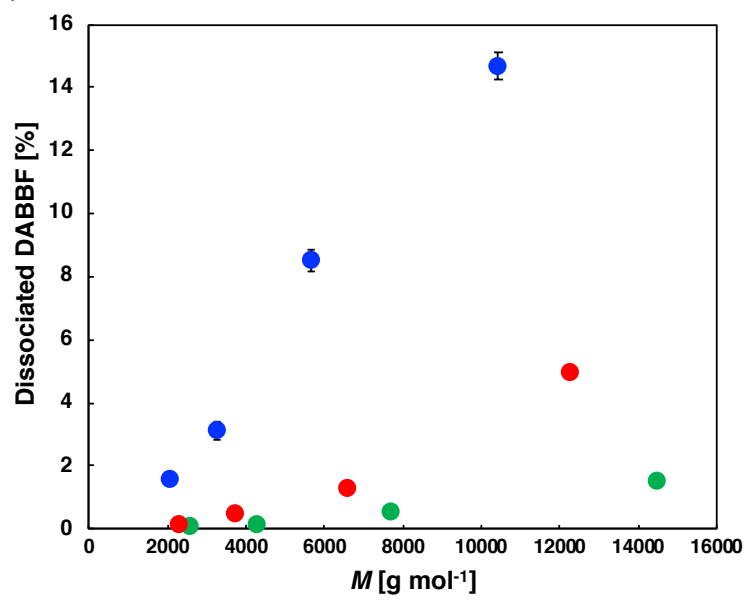

Figure S55. Activated DABBF ratio in MD plotted against a) the $T_{\mathrm{g}}$ and $\mathrm{b}$ ) the molecular weight.

\section{SI References}

(1) Oka, H.; Imato, K.; Sato, T.; Ohishi, T.; Goseki, R.; Otsuka, H. Enhancing Mechanochemical Activation in the Bulk State by Designing Polymer Architectures. ACS Macro Lett. 2016, 5 (10), 1124-1127.

(2) Leon, J. W.; Kawa, M.; Fréchet, J. M. J. Isophthalate Ester-Terminated Dendrimers: Versatile Nanoscopic Building Blocks with Readily Modifiable Surface Functionalities. J. Am. Chem. Soc. 1996, 118 (37), 8847-8859.

(3) Watabe, T.; Ishizuki, K.; Aoki, D.; Otsuka, H. Mechanochromic Dendrimers: The Relationship between 
Primary Structure and Mechanochromic Properties in the Bulk. Chem. Commun. 2019, 55 (48), 68316834.

(4) Emer, E.; Pfeifer, L.; Brown, J. M.; Gouverneur, V. Cis-Specific Hydrofluorination of Alkenylarenes under Palladium Catalysis through an Ionic Pathway. Angew. Chem. Int. Ed. 2014, 53 (16), 4181-4185. 